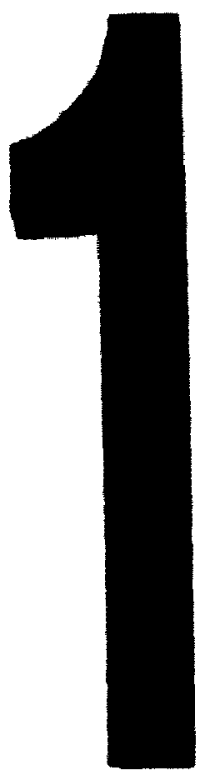

PM-1 31/2"X 4" PHOTOGRAPHIC MICROCOPY TARCET NES 1010a ANSI/1SO w2 EOUIVALENT

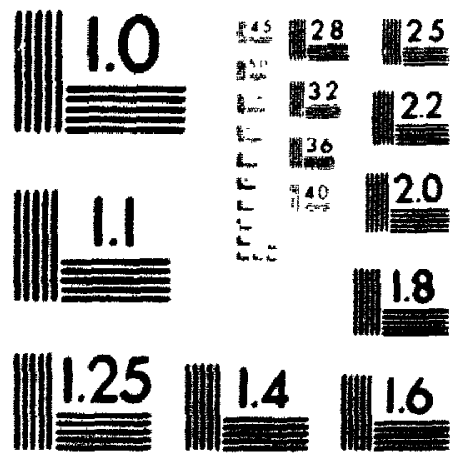


National Library

of Canada

Acquisilions and

Bibliographic Services Branch

395 Weilington Street

Ortawa. Ontario

KIA ONA
The quality of this microform is heavily dependent upon the quality of the original thesis submitted for microfilming. Every effort has been made to ensure the highest quality of reproduction possible.

If pages are missing, contact the university which granted the degree.

Some pages may have indistinct print especially if the original pages were typed with a poor typewriter ribbon or if the university sent us an inferior photocopy.

Reproduction in full or in part of this microform is governed by the Canadian Copyright Act, R.S.C. 1970, c. C-30, and subsequent amendments.
La qualité de cette microforme dépend grandement de la qualité de la thèse soumise au microfilmage. Nous avons tout fait pour assurer une qualité supérieure de reproduction.

S'il manque des pages, veuillez communiquer avec l'université qui a conféré le grade.

La qualité d'impression de certaines pages peut laisser à désirer, surtout si les pages originales ont été dactylographiées à l'aide d'un ruban usé ou si l'université nous a fait parvenir une photocopie de qualité inférieure.

La reproduction, même partielle, de cette microforme est soumise à la Loi canadienne sur le droit d'auteur, SRC 1970, c. C-30, et ses amendements subséquents. 


\title{
A Pipelined, Multi-Processor Architecture for a Connectionless Server for Broadband ISDN.
}

\author{
by \\ Daniel Omundsen, B.E.(Hons) (Elec) \\ University of Canterbury \\ Christchurch, New Zealand.
}

\author{
A thesis submitted to the \\ Faculty of Graduate Studies and Research \\ in partial fulfillment of the requirements \\ for the degree of \\ Master of Engineering
}

Ottawa-Carleton Institute for Electrical Engineering

Faculty of Engineering

Department of Systems and Computer Engineering

Carleton University

Ottawa, Ontario, Canada

Wednesday, 19 August 1992

- copyright

Danie! Omundsen. 1992 
Acquisitions and

Biblographic Services Branch

395 Wellington Street

Ottawa Ontario

KIA ONA
Direction ces acquisitions et

des services bibliographiques

395. rue Welington

Ottawa (Ontario)

KIA ON4
The author has granted an irrevocable non-exclusive licence allowing the National Library of Canada to reproduce, loan, distribute or sell copies of his/her thesis by any means and in any form or format, making this thesis available to interested persons.
L'auteur a accordé une licence irrévocable et non exclusive permettant à la Bibliothèque nationale du Canada de reproduire, prêter, distribuer ou vendre des copies de sa thèse de quelque manière et sous quelque forme que ce soit pour mettre des exemplaires de cette thèse à la disposition des personnes intéressées.

L'auteur conserve la propriété du droit d'auteur qui protège sa thèse. Ni la thèse ni des extraits substantiels de celle-ci ne doivent être imprimés ou autrement reproduits sans son autorisation.

ISBN $\quad 0-315-84129-X$ 
The undersigned recommend to the Faculty of Graduate Studies and Research acceptance of the thesis

"A Pipelined, Multi-Processor Architecture for a Connectionless Server for Broadband ISDN."

Submitted by

\section{Daniel Omundsen, B.E. (Elec)}

in partial fulfillment of the requirements

for the degree of Master of Engineering.
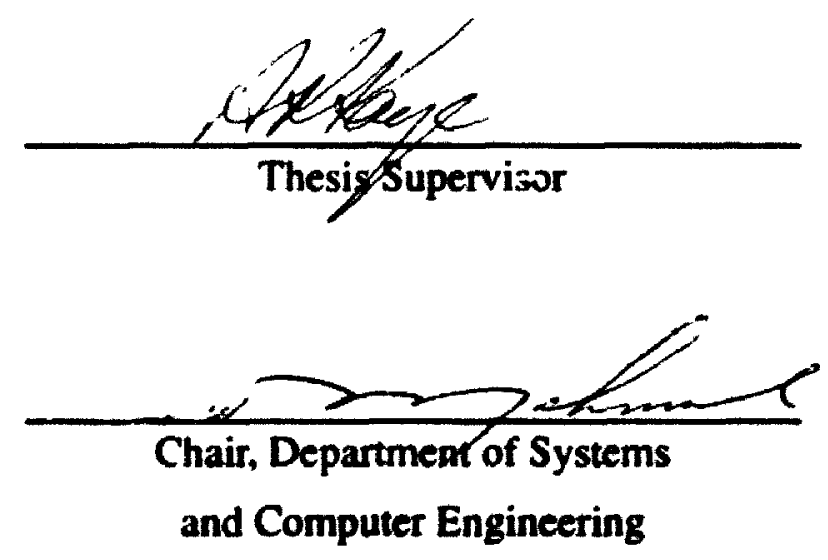

Carleton University,

Friday 18 September, 1992 


\section{$\underline{\text { Abstract }}$}

This thesis presents an architecture for a connectionless server for the diret support of connectionless data traffic in a Broadband ISDN network, using the CLNAP on top of the Type 4 AAL protocol. The high throughput required in the BISDN is achieved through the use of a multi-processor pipeline to process packet headers. A contribution of this work is the provision of customised hardware circuits, using content addressable memory, that perform much of the protocol processing overhead at high speed. The design separates cell and packet headers from the user data for high speed processing, routing packets without reassembly. The system is shown to be capable of operating under fully loaded links at both the standard bit rates of $155 \mathrm{Mbps}$ and $622 \mathrm{Mbps}$. From the results of this work. conclusions are drawn about the suitability of the Type 4 AAL for carrying connectionless data traffic. 


\section{Acknowledgments}

I would like to thank Professor A. Roger Kaye for his support and guidance throughout the completion of this work. The contribution of Professor Samy Mahmoud was also valuable.

The following organisations provided the financial backing which enabled this research to be carried out. To each of these $I$ also extend my appreciation and thanks.

- The John Templin Scholarship Trust (administered by the NZI Guardian Trust company of Christchurch, New Zealand).

- Telecom Corporation of New Zealand Ltd.

- The Telecommunications Research Institute of Ontario (TRIO).

- The National Sciences and Engineering Research Council of Canada (NSERC).

I would also particularly like to thank my parents, Dave and Anne Omundsen of Wanganui, New Zealand, and my in-laws, Rob and Jan Burrell of Runanga, New Zealand for their tremendous support and for the efforts that they have gone to, to ensure that this venture was a success.

Finally, my most special thanks extend to my wife, Helen, and son, Deane, for finding the strength to accompany me nearly halfway round the world, for sticking with it through both the good and the tough times, and for providing me with the invaluable support and encouragement that made all this possible. 
To Helen. 


\section{Table Of Contents}

Acceptance Sheet

ii

Abstract

iii

Acknowledgments

iv

List of Tables

$\mathbf{x}$

List of Figures

xi

Guide to Abbreviations

xiii

Chapter 1: Introduction.

1.I Background.

1.2 Objectives.

1.3 Literature Review,

1.4 Layout of the Thesis.

Chapter 2: The Broadhand ISDN Connectionless Data Service.

2.1 Introduction.

2.2 Providing a Connectionless Data Service in BISDN.

2.2.1 Connectionless Emulation using Switched Virtual Circuits.

2.2.2 Semi-Permanent Virtual Paths.

2.2.3 Variable Bandwidth Paths.

2.2.4 Connectionless Overlay Network

2.2.4.1 Operation of the CLSF.

2.2.5 Combining Advantages: A Hybrid Approach.

2.3 Transporting Data Over ATM Networks.

2.3.1 Protocol Specifications.

2.3.1.1 ATM Layer.

2.3.1.2 AAL Layer

2.3.1.3 SAR Sublayer. 
3.1 Initial Design Approach.

3.1.1 Single Processor system.

3.1.2 Multi-Processor system.

3.4.1 Operation of the ICU. 4I)

3.4.1.1 Functions common to all cells. $\quad 41$

3.4.1.2 BOM cell procedures. $\quad 42$

3.4.1.3 COM cell procedures. $\quad 42$

3.4.1.4 EOM cell procedures. $\quad 42$

3.4.1.5 System cell and error handling procedures.

3.4.2 Hardware Description. $\quad 44$

3.5 Cell RAM and Free Space List. 46

3.6 Message Reassembly Processor and Active Connection Table. 47

3.6.1 Active Connection Table format. 48

3.6.2 MRP Operation. 49

3.6.2.1 BOM procedures. 51

3.6.2.2 COM procedures. $\quad 52$

3.6.2.3 EOM procedures. $\quad 53$

3.6.2.4 Error Handling. $\quad 54$

3.6.3 Hardware Description. 56

3.7 Routing Processor (RP). 56

3.7.1 Functions of the RP. 58

3.7.1.1 Sequence of operations. 58

3.7.1.2 Routing Table Organisation. 62

3.8 Bandwidth Control Processor. $\quad 64$

3.8.1 Procedures Followed by the BCP. 65

3.8.1.1 Bandwidth Control Procedures.

3.8.1.2 Transmission Procedure. 68

3.8.1.3 Multicasting procedure. 
$\begin{array}{ll}3.8 .1 .4 \text { Abort cell procedures. } & \mathbf{7 0}\end{array}$

3.8.1.5 Setup and Maintenance Procedures. $\quad 70$

3.8.2 Hardware Description. 71

3.9 Outgoing Cell Unit. $\quad 72$

3.9.1 Procedures followed by the OCU. $\quad 72$

3.9.2 Hardware Description. $\quad 74$

$\begin{array}{ll}\text { Chapter 4: Experimental Results. } & 76\end{array}$

$\begin{array}{ll}4.1 \text { Approach Taken. } & 76\end{array}$

4.2 Default Parameter Values used.

4.2.1 Input Traftic Parameters.

4.2.2 Input/Output Parameters.

4.2.3 MRP Parameters. $\quad 79$

4.2.4 RP Parameters. $\quad 80$

4.2.5 BCP Parameters. $\quad 80$

$\begin{array}{ll}4.3 \text { Accuracy of the results. } & 81\end{array}$

4.4 Results.

4.4.1 Effect of the input traffic parameters. 85

4.4.1.1 Traffic Load. $\quad 85$

4.4.1.2 Burst Length. $\quad 88$

4.4.1.3 Cell Error Rate. $\quad 90$

4.4.1.4 Group Address Probability. 91

4.4.2 Effect of the MRP model parameters. $\quad 92$

4.4.3 Effect of the RP model parameters. 94

4.4.4 Effect of the BCP model parameters. 95

$\begin{array}{ll}4.5 \text { Conclusions. } & 98\end{array}$

$\begin{array}{ll}\text { Chapter 5: Modifications to the Design. } & 100\end{array}$

$\begin{array}{ll}5.1 \text { Processor Configurations. } & 100\end{array}$

5.2 Increasing System Capacity. $\quad 101$

5.3 Use of an altemative AAL Layer. 103 
Chapter 6: Conclusions.

Appendix A: Details of ICU Operation.

A.1 Input Control Tasks.

A.2 Transfer Control Tasks.

Appendix B: Details of the MRP Operation.

B.1 COM cell operation. $\quad 114$

B.2 BOM cell operation. $\quad 117$

B.3 EOM cell operation. 120

$\begin{array}{ll}\text { B.4 SSM cell operation. } & 121\end{array}$

B.5 ABORT cell operation. $\quad 122$

$\begin{array}{ll}\text { B.6 Error Handling procedures. } & 124\end{array}$

B.7 Setup and Maintenance Procedures. $\quad 125$

Appendix C: Details of the OCU operation. $\quad 127$

$\begin{array}{ll}\text { C.I Normal cell procedures. } & 127\end{array}$

C.2 System/Idle/Abort cell procedures. $\quad 128$

Appendix D: The Simulation Model. 130

D.1 Input Traffic Model. 131

D.2 Input/Output Model. $\quad 134$

D.2.1 Statistical Multiplexing. $\quad 134$

D.2.2 ICU model. $\quad 135$

$\begin{array}{ll}\text { D.2.3 Output Model. } & 135\end{array}$

D.3 MRP Model. $\quad 136$

D.4 RP Model. $\quad 137$

D.5 BCP Model. 138

D.5.1 Processor Model. 138

D.5.2 BCP hardware model. 139

$\begin{array}{lr}\text { References. } & 141\end{array}$ 


\section{List of Tables}

Table 4-1 Input traffic parameter values

Table 4-2 Input and output parameters.

Table 4-3 MRP Cell Processing Times

Table 4-4 Routing Processor execution times.

Table 4-5 Parameters used in BCP processor model.

Table 4-6 Results output by the simulation program.

Table 4-7 Processing Linits for the MRP

Table 4-8 Limits on RP Processing Time.

Table 4-9 Operating Limits on BCP algorithm. 


\section{List of Figures}

Figure 2-1 Connectionless service using a network of meshed virtual connections. 12

Figure 2-2 Connectionless service using a logical overlay network.

$\begin{array}{lll}\text { Figure 2-3 } & \text { Physical location of CLSF } & 17\end{array}$

Figure 2-4 Provision of a connectionless data overlay network within the B-ISDN. I9

Figure 2-5 BISDN Protocol Reference Model for Connectionless Data. 21

Figure 2-6 LAN-to-LAN Protocol Stack for the Direct Connectionless Service. 22

Figure 2-7 Format of ATM Cell.

Figure 2-8 SAR-PDU Format.

Figure 2-9 Format of the CPCS-PDU 26

Figure 2-10 Format of the CLNAP-PDU. $\quad 2 x$

Figure 3-1 Initial Design: Single Processor Basic Architecture. 32

Figure 3-2 Initial Design: Multi Processor Basic Architecture

Figure 3-3 Functional Architecture of the CLSF. 37

Figure 3-4 Format of incoming cells

Figure 3-5 Format of Header Queue entries.

Figure 3-6 Functional Architecture of ICU $\quad 4.5$

Figure 3-7 Format of Active Connection Table entries $4 \times$

Figure 3-8 CAM mask used to identify messages. 49

Figure 3-9 Organisation of a portion of the message part of the Header RAM. S0)

Figure 3-10 Format of QOS queue entry $\quad 52$

Figure 3-11 Format of message list block.

Figure 3-12 Functional Diagram of Message Reassembly Processor. 57

Figure 3-13 Outgoing Queue portion of the Header RAM 59

Figure 3-14 Examples of circuit list entries. (x)

Figure 3-15 Format of Connection List Entry (x)

Figure 3-16 Outgoing Connection Queue Entry.

Figure 3-17 Routing Tree Organisation.

Figure 3-18 Outgoing Header Queue Entries

Figure 3-19 System Queue Bandwidth Specification Entry. 71

Figure 3-20 Functional Diagram of the Bandwidth Control Processor. 71

Figure 3-21 Functional architecture of the Outgoing Cell Unit. 75 
Figure 4-1 Variation of processor usage with traffic load. 85

Figure 4-2 Variation of Input and Output Queue lengths with traffic load. 86

Figure 4-3 Variation of Quality of Service Queue lengths with traffic load. $\quad 87$

Figure 4-4 Variation of connection queues and RAM contents with traffic load. 87

Figure 4-5 Variation of Packet delay with traffic load 88

Figure 4-6 Effect of Burst Length on MRP and RP Load. 89

Figure 4-7 Variation of system contents and packet delays with burst length. $\quad 89$

Figure 4-8 Effect of cell error rate on traffic and MRP usage. $\quad 90$

Figure 4-9 Effect of changing output load due to cell error rate. 91

Figure 4-10 Result of increasing MRP BOM time on link loading and MRP usage. 93

Figure 4-11 Effect of RP processing time on link traffic and RP utilisation. 94

Figure 4-12 Effect of BCP processing time on output traffic and BCP utilisation 96

Figure 4-13 Effect of BCP scan algorithm on processor usage and output queue. 97

Figure 4-14 Effect of BCP scan size on packet delays and cells in system. 98

$\begin{array}{lll}\text { Figure 5-1 Arrangement of virtual circuits for a multi-link system } & 103\end{array}$

Figure B-1 Timing Diagram for COM cell processing in MRP 117

$\begin{array}{lll}\text { Figure B-2 } & \text { Timing Diagram for BOM cell processing in MRP } & 120\end{array}$

Figure B-3 Timing Diagram for EOM cell processing in MRP 121

$\begin{array}{lll}\text { Figure B-4 } & \text { Timing Diagram for ABORT cell processing in MRP } & 124\end{array}$ 


\section{Guide to Abbreviations}

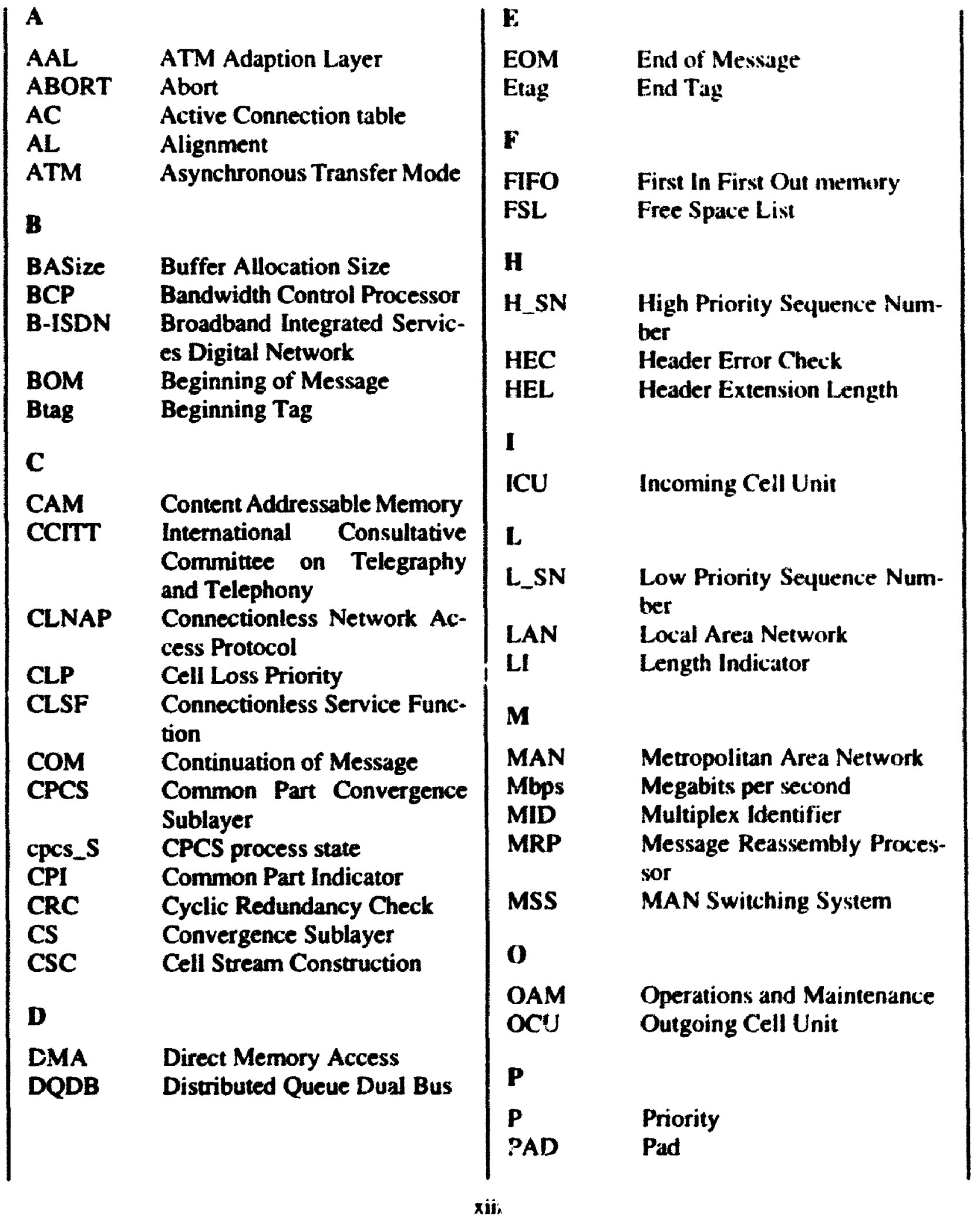




$\begin{array}{|ll|}\text { PDU } & \text { Protocol Data Unit } \\ \text { PT } & \text { Payload Type } \\ \text { QOS } & \\ \text { R } & \text { Quality of Service } \\ \text { RAM } & \\ \text { rcv_BASize } & \text { Receiver BA size } \\ \text { rcv_BEtag } & \text { Reciver BEtag } \\ \text { rCv_size } & \text { Received size } \\ \text { rcv_SN } & \text { Receiver sequence number } \\ \text { REASS } & \text { Reassemble } \\ \text { RISC } & \text { Reduced Instruction Set Com- } \\ \text { RP } & \text { puter } \\ \text { S } & \text { Routing Processor } \\ \text { SAR } & \\ & \text { Segmentation and Reassem- } \\ \text { Sar_S } & \text { bly } \\ \text { SMDS } & \text { SAR process state } \\ \text { Switched Multimegabit Data } \\ \text { SN } & \text { Service } \\ \text { SONET } & \text { Sequence Number } \\ \text { Synchronous Optical NET- } & \text { work } \\ \text { SSCS } & \text { Service Specific Convergence } \\ \text { SSM } & \text { Sublayer } \\ \text { ST } & \text { Single Segment Message } \\ \text { V } & \text { Segment Type } \\ \text { VCI } & \text { Virtual Circuit Identifier } \\ \text { VPI } & \text { Virtual Path Identifier } \\ & \end{array}$




\section{Chapter 1: Introduction.}

\subsection{Background.}

The current trend in telecommunications research is leading towards the development of a new type of public switching system. the Broadband Integrated Services Digital Network (BISDN). The BISDN is expected to eventually replace existing public networks with a single system capable of carrying voice, high fidelity sound, still images, full motion video, computer data, distributed processing. multimedia combinations of the above, and any other kind of high bandwidth store and forward or real time communicaltions. It is an evolution of the ISDN concept (that is being brought into service by telecommunications companies today) into the area of broadband communications.

The introduction of the BISDN is expected to take place gradually, with existing equipment being replaced as it wears out and new services becoming available as soon as

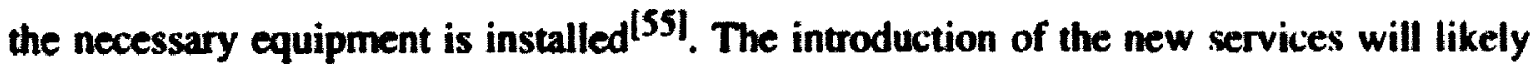
be driven by customer demand, so that services that can take advantage of already existing customer equipment will be implemented first ${ }^{[15]}$.

One of the first services to be supported by the BISDN will be the carrying of computer data traffic, in particular traffic from local or metropolitan area networks (LANs or MANs). This is because the high bandwidth traffic carried by the BISDN (up to 150 or $600 \mathrm{Mbps}$ ) requires optical fibre conr $+c$ ctions, and high capacity optical tibre networks such as the Fibre Distributed Data Interface (FDDI) and Distributed Queue Dual Bus (DQDB) networks are already becoming common ${ }^{[26]}$. The existence of such networks generates the demand for high quality, high bandwidth interconnection between them that the BISDN can supply. It is anticipated that the evolution of data communications networks will proceed from LANs and MANs to BISDN ${ }^{[3,37 !}$. 
Chapter 1: Introduction.

Because of this, it is important that the method by which the BISDN will carry data traffic from computer networks (known as connectionless data traffic) be developed as quickly as possible. As will be discussed in Chapter 2 two possible methods have been identifier, known as the direct and indirect BISDN connectionless data services. The indirect method involves using the BISDN as simply a transmission medium to carry the information from one point to another, while the direct service requires that the BISDN play an active role in the routing of the data ${ }^{[7]}$.

The implementation of the direct BISDN connectionless data service requires that an entity within the public network receives packets from the source LAN or MAN and routes them to their destination based on addressing information contained within the packets. The entity is known as a connectionless server, and although its function is similar to a packet switch the way in which it operates is distinctly different.

It is the intention of this thesis to present a design for such a server, that could be used within a BISDN to provide the direct connectionless service to both existing and future types of computer data traffic.

\subsection{Objectives.}

The connectionless server (referred to in the BISDN service definition as a Connectionless Service Function or CLSF) should be capable of performing the routing functions at the extremely high data rates used in a BISDN network. It should be able to handle a number of packets multiplexed together on a single physical link simultaneously, and it should output the packets in a way that conforms with the BISDN data format and traffic characteristic specifications.

The object of this research was to design a hardware system that meets these requirements. It was not intended to produce a detailed set of plans, but rather to come up with a 
functional architecture that could be built using existing technology. The design forms a basis upon which a CLSF device for use in the BISDN could be built.

To test the validity of the design. computer simulation is used to examine the performance of the system under different conditions of input traftic and design parameters. From the results of these simulations, it is possible both to determine whether or not the design is feasible, and to estimate the performance in terms of maximum throughput and packet delay that are likely to be encountered in a system based on the design principles. The simulation results can also be used to fine tune the design so as to improve performance and reduce hardware costs.

The design is based upon the current state of standardisation of the BISDN, and incorporates the concept of the CLSF as implied by the standards and as suggested by previously published papers related to data traffic in the BISDN. Future changes in the BISDN standards, particularly those relating to the Conriectisnless Network Access Protocol (CLNAP) and to the use of an additional simplified ATM Adaptation Layer (AAL) for data traffic, may necessitate minor changes to the details of this design. However, the overall principles of operation remain the same.

\subsection{Literature Review.}

In order to avoid the long negotiation and standardisation delays that plagued the introduction of ISDN, international standards bodies such as the International Telegraph and Telephone Consultative Committee (CCITT) are working rapidly to produce a set of standards that characterise and define the BISDN17-10.51-53!. This activity has inspired a large body of research into various aspects of the BISDN.

To the best knowledge of the author, there has not been to this date any published work specifically regarding connectionless server architectures. This thesis, therefore, must draw on the results of work in other related areas. This section summarises a few of the 
published documents that contributed to the production of this work. Readers unfamiliar with the BISDN connectionless data service may wish to read Chapter 2 first, in order to gain a better understanding of the concepts introduced by the papers.

A number of papers have been written which describe the operation of the BISDN direct connectionless service, and propose network arrangements to support it. The most important of these are the CCITT standards mentioned above, which represent the international body of agreement about the BISDN and which are still in the process of review. Other, earlier papers present a more easily read description of the connectionless service.

Many of the earlier papers, such as the one by S. lisaku and $M$. Ishikura ${ }^{[25]}$ or by Y. Ujihashi et al ${ }^{[54]}$ simply discuss the direct and indirect connectionless services, and propose network or gateway architectures to support them. The use of a network of CLSFs interconnected by semi-permanent paths as described in these papers has since become the accepted direct method.

B. Amin-Salehi and D. R. Spears wrote a paper in 1989 which reviews the use of the BISDN to carry both connectionless and connection-oriented data ${ }^{[1]}$. They describe the physical location and use of a CLSF, and illustrate a possible series of steps that the CLSF must follow. They also suggest the use of a reassembly table, an E.164 address translation table, and a packet reassembly area to make possible the switching of data.

The paper by M. Lobjinski et al ${ }^{[36]}$ discusses the difference between the direct and indirect service, and conclude that the direct service is more efficient in terms of bandwidth. They also consider the problem of traffic multiplexing, and point out that a logical output queue is required within the CLSF for each outgoing virtual circuit connection, to ensure that the outgoing bandwidth allocations are not exceeded.

T. Van Landegem and P. Peschi ${ }^{[32]}$ consider the BISDN connectionless service as a whole. and examine the configuration of the connectionless overlay network. This includes both systems using the direct service, and systems that use the indirect service. 
They consider the control of this network, and point out that the CLSF control can be split into two parts - that responsible for the routing of data and that responsible for the maintenance of the overlay network. They also point out that the control infrastructure will take advantage of the extreme flexibility of the BISDN to be able to recontigure the overlay network, including providing alternate routes in times of congestion and allowing for dynamic adjustment of reserved connection bandwidths.

G. Fioretto $e a^{[19]}$ in their discussion of the connectionless service mention that it is possible for the CLSF to begin transmission of a packet before it has been completely received, in order to avoid excessive data buildup within the system. Their interpretation of the CLSF functionality, however, makes the assumption that the CLSF simply extracts the destination address from the data, then relies on the BISDN call routing control to select an appropriate outgoing virtual circuit, and to reserve bandwidth on that circuit. This process would place an unduly heavy load on the BISDN, which is also busy handling other types of traffic.

Other papers that are useful to the purpose of this work are related to the hardware design. B. S. Davie presents a hardware design for an interface between a host computer and an ATM network ${ }^{[17]}$. He uses special purpose hardware controlled by finite state machines, along with two Intel $8096033 \mathrm{Mhz}$ RISC microprocessor subsystems, to transfer data between a host computer and a 622 Mbps ATM link. The hardware is responsible for transferring the cell payloads to and from the host, while the processors are used to make control decisions about where in the host RAM to place or retrieve the data. FIFO queues are used to decouple parts of the system that run at different speeds from one another. Due to speed constraints, the available VCl address space is limited to 16 bits.

C. B. S. Traw and J. M. Smith have designed and built a host interface that transfers data to and from a $155 \mathrm{Mbps}$ ATM system ${ }^{[49]}$.They separate incoming cell data from the cell headers, and use a CAM lookup on the cell headers to determine what connection the cell belongs to. The messages are reassembled by placing pointers to the cell bodies in a 
Chapter 1: Introduction.

series of linked lists stored in RAM. Because the system is designed to interface to a workstation, the number of active virtual circuits is limited to 256.

The paper by T. B. Pei and C. Zukowski ${ }^{[42]}$ illustrates how the problem of routing data can be easily implemented in RAM by use of a tree structured approach, which they term a "trie" (from the word "retrieval"). The trie approach examines a destination address in sections, which corresponds quite well with the hierarchical nature of the E.164 addressing scheme used in the BISDN.

\subsection{Layout of the Thesis.}

The remainder of this thesis is organised into five chapters plus appendices. Chapter Two introduces the BISDN connectionless data service, by first describing how the BISDN may be used to carry data traffic. A number of options for adapting the connection-oriented BISDN for connectionless data traffic are given, and their suitability for different traffic types are analysed. This is followed by a detailed description of the BISDN protocols as they relate to the direct connectionless data service.

Chapter Three presents the CLSF design itself. Two initial design attempts are given to introduce the reader to the background and concepts used in the final system. This is followed by an overview of the system operation, before delving into the details of operation of the different system components. Hardware diagrams of the components are presented, but a description of the sequence of operations in those which use special purpose devices are left for the appendices.

Chapter Four details the computer simulation experiment used to verify the design. Firstly, the way in which the system was modelled is discussed and the default system parameters used in the experiment are presented. A description of the procedures used to reduce errors in the results is given. Next, important results from the simulation runs are presented in graphical form. Conclusions about the conditions of validity of the design are 
Chapter I: Introduction.

then drawn from those results. The details of operation of the simulation program are presented in an appendix.

Chapter Five explains how the results from the simulation can be used to suggest modifications to the design that cut down on hardware costs. Ways in which the system capac:ity can be extended are given. as well as suggestions for future work on this system. The use of the new Type 5 AAL in this system is discussed.

Finally, Chapter Six summarises the major conclusions that can be drawn from this work. 


\section{Chapter 2: The Broadband ISDN Connectionless Data Service.}

\subsection{Introduction.}

The Broadband Integrated Services Digital Network is based on a transmission method known as Asynchronous Transfer Mode (ATM). The ATM principle involves breaking up the data from a source into a series of small packets or cells, then using statistical multiplexing to place the cells from a number of sources on to a single physical link. The cells contain a header which identifies which logical or virtual connection the data belongs to. Readers who wish to know more about ATM are advised to consult the book by $M$. de Prycker ${ }^{[44]}$ for an introduction to the topic.

In general, two alternative strategies exist for establishing data communications over a network. Communication may be either connection-oriented or connectionless, where the difference is in how a communication session is initiated.

In a connection oriented data session, before any information is sent some sort of control procedure is carried out to establish a logical connection through the network. This path is effectively an association between the two endpoints, which determines the route to be taken by the data. Once the connection has been established, the data to be sent is forwarded by the network to the destination, and further control procedures remove the connection. The proposed ATM network, as a future telephony system, is by definition connection oriented.

The altemative way to transport data is the connectionless method, which works without call setup procedures. Instead, a terminal with dats to send creates a data packet which contains the receiver's network address, and simply places it in the network. Mechanisms 
in the network ensure that the packet reaches its destination by examining the address. This technique is the basis of computer communication via local area networks.

Obviously, this fundamental difference in methodology creates some problems when we try to use ATM to carry data traffic that originates on a connectionless network such as a LAN. This section examines those problems, and explains the solutions that are likely to be used in future BISDN networks. The implementation of one of these solutions forms the goal of this thesis.

There are two main issues to consider when investigating the mechanism of using ATM networks to carry data traffic, and they are presented in the following sections. Section 2.2 explains the problem of how to provide a connectionless service in a connection-oriented network, and details possible solutions including that which is addressed by this thesis. Section 2.3 examines the protocol aspects of using Asynchronous Transfer Mode to carry data traffic, and presents the current standards that are used as a basis for this work.

\subsection{Providing a Connectionless Data Service in BISDN.}

Because an ATM network is a connection-oriented network, traftic is routed according to a set path or connection that is established some time prior to transmission and cleared some time afterwards.

In order for connectionless data to be routed correctly to its destination using ATM, some arrangement must exist so that the network can treat it as it would any other type of traffic, i.e. through the use of connections. The network must still appear connectionless from the point of view of the end user. Data sent from the user terminal must be forwarded without the user undergoing any call setup procedures.

A number of possible methods exist for emulating connectionless data transfer over an ATM network ${ }^{[32]}$. The methods have a number of advantages and disadvantages with 
Chapter 2: The Broalband ISDN Connectionless Data Service.

respect to speed, equipment complexity and processing load and are suited to different types of traffic. The alternatives are summarised in the following subsections.

\subsubsection{Connectionless Emulation using Switched Virtual Circuits.}

The first and most obvious way to carry connectionless data over ATM involves automatically establishing and releasing a connection when there is data to be sent. This function can be performed by the ATM interface or bridge.

The bridge/ATM interface inspects the destination address contained in the header of each outgoing data packet it receives. It then determines the location of the destination using existing LAN techniques (such as learning algorithms, server queries, etc.), and con-

sult an internal table to determine the B-ISDN address (telephone number) of the LAN to which the packet is to be sent. The interface contacts the ATM network and negotiates the setup of a circuit connection through the network to that address (i.e. it dials the number). A virtual circuit (VC) between the endpoints is established, and the bridge is assigned a VC identifier to use. The data packet is then forwarded as described in Section 2.3. At the end of transmission, the bridge informs the network and the call is cleared (it hangs up).

This method can also be used by a terminal or workstation which is connected directly to the ATM network. It is possible for the terminal to "dial up" a host or a LAN as described above in a manner analogous to the use of today's modems. In this case rather than being cleared at the end of each packet the call will be cleared at the end of the session. Also, the connection will be two-way. This is different from a true connection-oriented communications session in the sense that from the point of view of the host, the terminal appears to be attached to a connectionless LAN.

The biggest disadvantage of this method is the potential call processing load on the ATM network. As the speed of the LAN increases the number of packets per second being sent over the network can get very high. resulting in a large number of call requests being 
processed by the network. far in excess of that generated by normal telephone traftic. This can have serious consequences on overall network performance.

A partial remedy to this problem is to leave the circuit open for some period of time after the end of the packet. The circuit will only be cleared when it has been idle for the pre-determined period. In this way successive packets intended for the same destination are forwarded without additional call setup procedures.

Another problem arises when the speed of the attached LAN gets very high. During the call setup phase, outgoing data must be stored in a buffer while the call is being established. This could take a significant amount of time, for example $3100 \mathrm{~ms}$ in a very fast ATM network ${ }^{[16]}$. In the worst case scenario, all the traftic on the LAN during the call setup phase would be destined for the ATM network. Thus, the bridge must contain a buffer big enough to store all the data transmitted during the call setup time $\left.\right|^{|43|}$. For a 10 Mbps LAN, the buffer requirement would be 3 Mbits using the above setup time. The problem gets worse as the LAN speed increases.

It can be seen from the above arguments that providing an ATM connectionless service in this way is not very suitable for systems with high data speeds or righ outgoing packet rates. This method is more suited for low speed LANs with few outgoing packets and for individual terminals.

\subsubsection{Semi-Permanent Virtual Paths.}

The first step towards solving some of the problems with the above technique involves removing the need for multiple call setup procedures by leavisig the connections open. This is a special case of the time-out solution mentioned above where the time out period is very long.

With this method, a permanent or semi-permanent call between end stations is set up when the user initially subscribes to the service. The call remains open until the user no 
longer requires the connection sr until he stops paying his bills! This is equivalent to the "leased line" services in use today.

The result is a network of virtual connections between all desired pairs of endpoints attached to the network. This is illustrated in Figure 2-1.

ATM Network

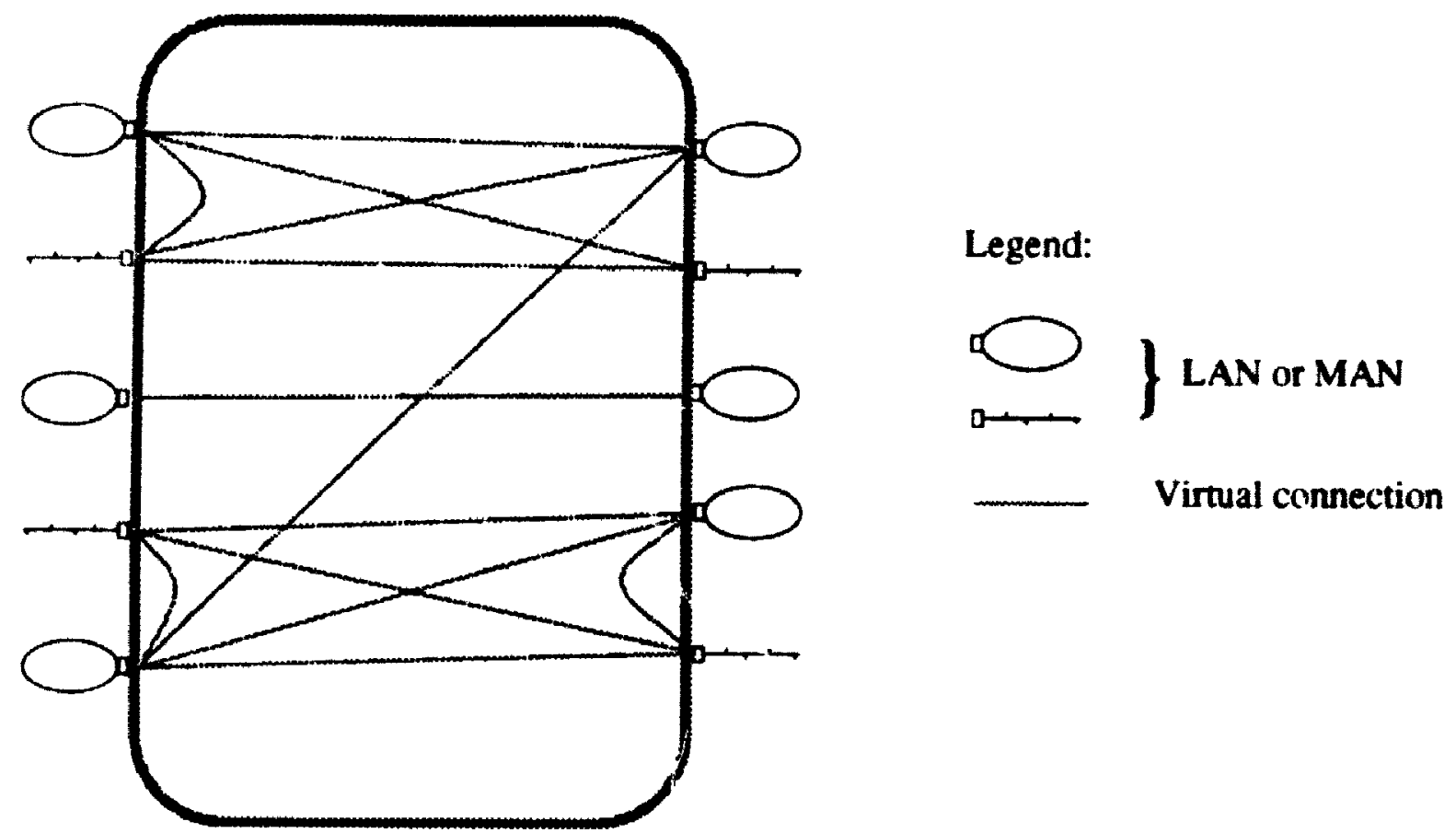

Figure 2-1 Connectionless service using a networix of meshed virtual connections.

Now there is no requirement for a large buffer in the bridge, as there is no call setup delay. Outgoing data packets are forwarded immediately on the existing connections. The call processing load on the network is minimal, as calls are rarely changed. This method is also superior to leased line services in that the connection need not be set up by the network operator, but could be done automatically by user equipment facilitating automatic network reconfiguration in large systems.

However. problems now arise from the network point of view as the number of attached stations increases. As each connection is established, a set of parameters relating to the peak bit rate, average bit rate and "burstiness" of the connection is negotiated with the network. The network allocates portions of its resources according to the agreed 
parameters. Thus, when the network is deciding whether or not to accept a call of any type (e.g. a voice or video call), it must take into account the amount allocatted to data calls. whether or not the circuit is being used at that point in time. Needless to say. the users will be charged according to the amount allocated to them. regardless of their usage of the circuit.

Thus, for financial reasons, it is unlikely that users who only occasionally make use their connections or whose usage fluctuates significantly over larger periods of time (say hourly) will wish to make use of this method. As a result it is more suitable for large systems such as MANs, with high traffic levels that don't fluctuate as significantly. This is true also from the network point of view, as established connections that aren 't being used represer+ inefficient utilisation of resources.

\subsubsection{Variable Bandwidth Paths.}

The above two methods cater for very small and very large systems respectively. In order to allow for medium-sized systems, which includes most small company networks, some modifications to the permanent paths method can be made. This involves the concept of "sleeping" connections.

A sleeping connection is one where a logical connection exists but no resources fother than the virtual circuit/virtual path resource) are allocated to the call. When there is data to send, the appropriate resources are allocated. More generally, the bandwidth allociation for the call fluctuates according to the load.

The exact method by which this is done is a subject currently under investigation. At least two alternatives exist ${ }^{[16]}$, and many others are possible.

The basic idea behind these methods is that the network gateway is continually monitoring the amount of traffic passing into the ATM network. As the traffic level alters over time, the gateway responds to the changing conditions by sending control messages to the 
Chapter 2: The Broadband ISDN Connectionless Data Service.

ATM network, requesting an adjustment to the agreed traffic parameter settings of the connection. It is one of the features of the BISDN that parameters of the call, such as the agreed mean and peak bandwidth settings, may be altered during the course of the call.

By doing this, the amount of network resources consumed (and the corresponding charges) are kept proportional to the actual traffic levels on the connection.

It is likely that the majority of connectionless data users will, at least in the short term, use some sort of variable bandwidth approach. This type of system caters for the needs of the majority of medium to large networks, and may even replace the use of fixed rate channels. As a result, it is a topic that is currently receiving research attention, and for which there is plenty of room for new suggestions.

\subsubsection{Connectionless Overlay Network}

Ali of the above methods are suitable for small scale (i.e. limited coinectivity) data networks, but share 1 common problem when the size and scale of the network increases that of network complexity. As more and more elements are added, the number of virtual circuit connections required to maintain adequate connectivity becomes much greater. As the network connectivity increases, so too does the problem of routing data.

Using the above techniques alone, the ATM network can be viewed as merely a transmission system within the data netw'ork. The traditional problems of address resolution, optimal route finding and fault control are handled with techniques that are in use today, by intelligent bridges and routers within the data network. These methods become unwieldy as the complexity of the network and the number of attached stations become very large.

The problem exists even in today's larger networks such as the relatively new Distributed Queue Dual Bus (DQDB) based MANs. A solution that is currently becoming available is Bellcore's Switched Multimegabit Data Service (SMDS) ${ }^{[24.47]}$. It addresses the 
issue of routing in complex broadband data networks. with special allowance made for public networks, and is intended to be initially available in a MAN environment. The key concepts include the use of CCITT E.164 addresses', a common interface protocol for interconnecting heterogenous networks, and the provision of routing functions or switching systems which are external to the customer network.

A similar such system is appropriate for use in an ATM environment. Rather than hav. ing a unique virtual path between each pair of connected network gateways, it is better to form a network of virtual paths connecting sets of connectionless switching nodes within the ATM network. In this way a separate data switching subnetwork is formed within the ATM network, as an overlay of logical connections. The switching nodes, or connectionless service functions (CLSFs), take over the problem of data routing from the gateways. The CCITT defines this approach in Recommendation I.211 as "direct support of the BISDN connectionless data service"[7] (as opposed to "indirect support" as described in the previous two sections). This concept is illustrated in Figure 2-2.

Each network gateway is connected only to the nearest CLSF. The data packets sent out by the gateway contain the destination E.164 address (telephone number), which the CLSF uses to route the packets either to another CLSF along a high usage route or to another local gateway.

As a result, the complexity of the gateway's operation is reduced. Instead of having to keep track of least cost routes to every station in the large data network, it only needs to keep a record of the telephone numbers or the E.164 addresses of the LAN connected to each station. As far as the gateway is concerned, it now has a one-hop connection to each LAN connected to the ATM network.

1. CCrTT Recommendation E.164 specifies the international telephune numbering format to be used in ISLN networks. ${ }^{|6|}$ Thus the use of E.164 addresses in a data network context makes it easy wo interwork with an ISUN type lelephone system. 


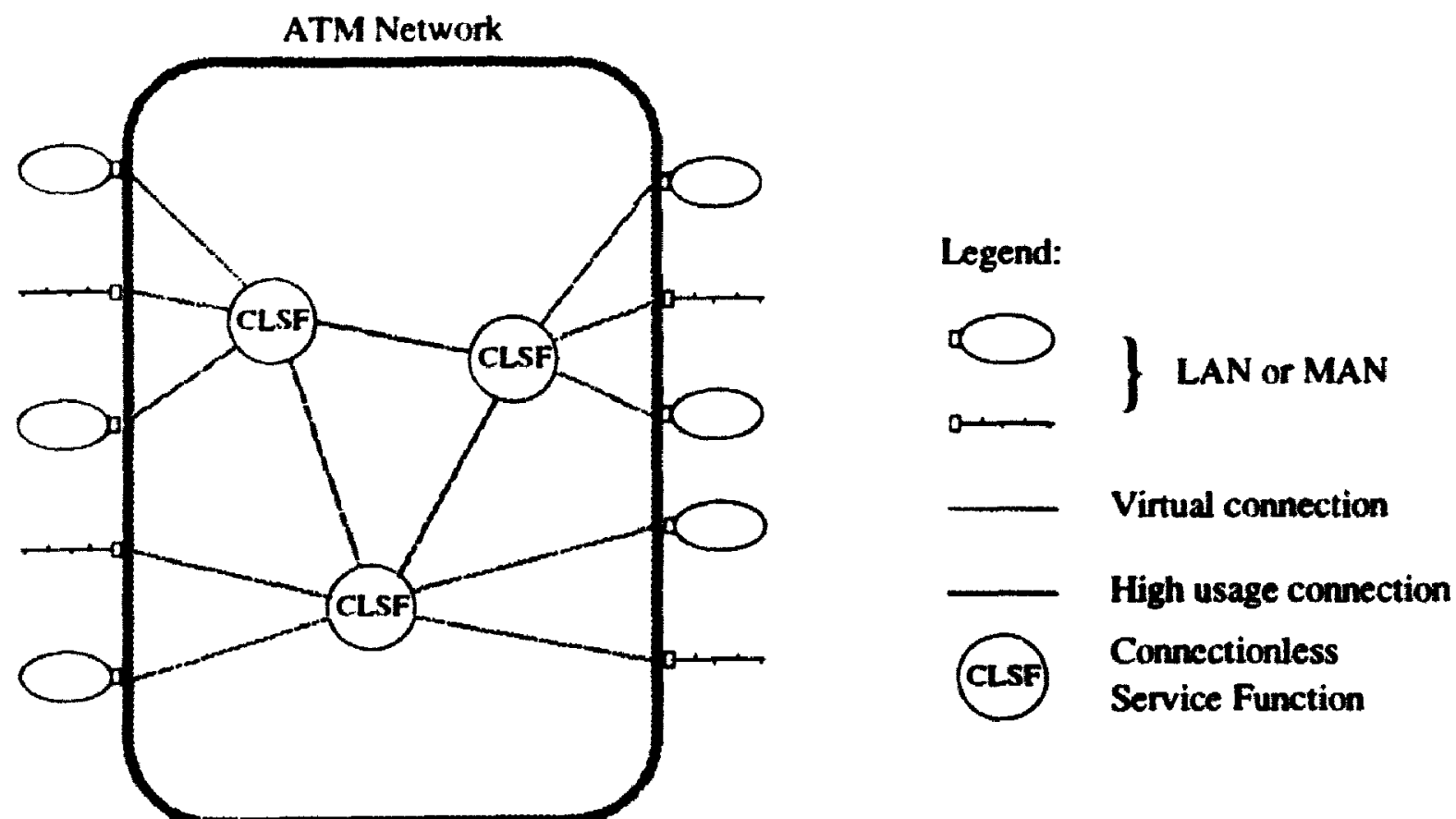

Figure 2-2 Connectionless service using a logical overlay network.

The routing function of the CLSF is also simpler than that of a normal router, as it only needs to keep track of the status of the virtual high usage network. The hierarchical nature of the E.164 addressing scheme, and the fact that it is common to the ATM network, means that the destination address can uniquely determine the destination CLSF. The sending CLSF needs to know only how to get to the destination CLSF, and to its local terminals.

Another advantage of this scheme is that many data connections are multiplexed together on the high usage routes. This takes maximum advantage of the benefits of statistically multiplexing very bursty sources. The net effect of putting many bursty sources together is to smooth the traffic, making fuller use of the bandwidth capacity of the link.

This architecture also makes possible specialised applications such as electronic mail and inter-company file transfer. Data can be sent between different company networks that would never normally be connected. Because of the security implications, this feature must be strictly controlled. 
Because of the processing required at the CLSF, there may be a practical limit to the speed at which data can be sent through the system. Also, there is no sense in connecting gateways to the overlay network if their traffic is all headed for the same destination. particularly if it is high-speed. This is simply adding unnecessary processing load to the system.

The connectionless overlay method is particularly suited to the interconnection of low and medium speed data networks with a number of desired destinations. It is not suited for high speed traffic with a single destination.

\subsubsection{Operation of the CLSF.}

In order to understand how the CLSF actually works, it is important to remember the context in which it operates. Although it is effectively routing data, the CLSF is a separate entity from the ATM switch. It has a number of bidirectional virtual circuit or virtual path connections between itself and some data users, and between itself and other CLSFs. Incoming data from one virtual connection is sent out on another, according to the routing decisions it makes. Figure 2-3 illustrates this relationship.

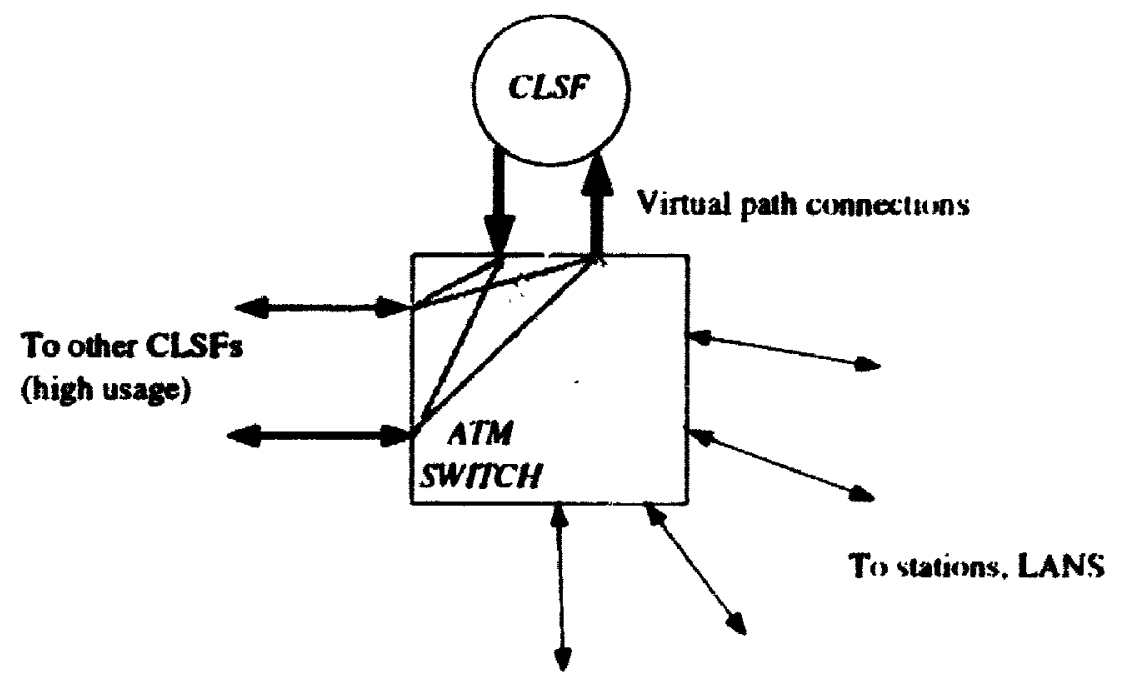

Figure 2-3 Physical location of CLSF

In order to switch a cell from an input conrection to an output connection, the CLSF simply has to give the cell new virtual circuit/virtual path identifiers (VCI/VPI numbers) 
and place it on an outgoing physical link. The ATM switch does the actual routing of the cells towards their destination based on the new VCl. Thus, the CLSF can be thought of as a $\mathrm{VCl}$ translator.

This differs from the current MAN based implementation of SMDS, where the cells are routed by a MAN Switching System (MSS) ${ }^{[2 A]}$ which does perform physical switching of the data. The CLSF takes full advantage of the benefits of operating in an ATM environment. by utilising the features of the underlying ATM network to simplify its own operation.

The result is that the physical complexity of the CLSF may be much simpler than conventional packet switches.

\subsubsection{Combining Advantages: A Hybrid Approach.}

Each of the aforementioned techniques has its advantages and disadvantages for different types of traffic. In order to provide the most effective use of the B-ISDN for a wide variety of traftic types, it is possible to combine all the solutions proposed above into a hybrid system. Each type of connection would then use the method most suited to it, and to the users needs. In order to cater effectively for all possible combinations of user equipment. each of these methods should be present in some degree.

Where MANs and other high traffic entities are to be connected, it is best to use a direct connection between end points. This connection can be either fixed or variable bandwidth, depending on the expected traffic levels and their changeability over time. It could also be useful for the MAN to have a CLSF connection for applications such as electronic mail, and to enable access to systems used infrequently.

Where LANs are to be connected only to a MAN, variable bandwidth direct connections are suitable. This applies regardless of the LAN size. 
A MAN-like network can be built up entirely within the BISDN. In this case. the CLSF is used exclusively, and each participating LAN has its own connection to the nearest CLSF. The CLSF takes over the MAN routing functions currently provided by systems such as SMDS.

LAN to CLSF connections should all be variable bandwidth. If the LAN is particularly small, then connections can be dial-up. In fact. the most efticient way to use long distance dial-up connections (between LANs in a connectionless environment, not connection-oriented) is via the CLSF. This is because the connection establishment procedure need only go as far as the nearest CLSF, and the rest of the way is pre-established.

Terminals may also connect to the CLSF through dial-up connections in order to access a remote LAN, or to send and receive electronic mail. The CCITT states in Recommendation I.211 that the establishment of virtual connections at the commencement of a connectionless service session is an option when using the direct BISDN connectionless service ${ }^{[\$ 1]}$.

Figure 2-4 illustrates how such a hybrid system could look.

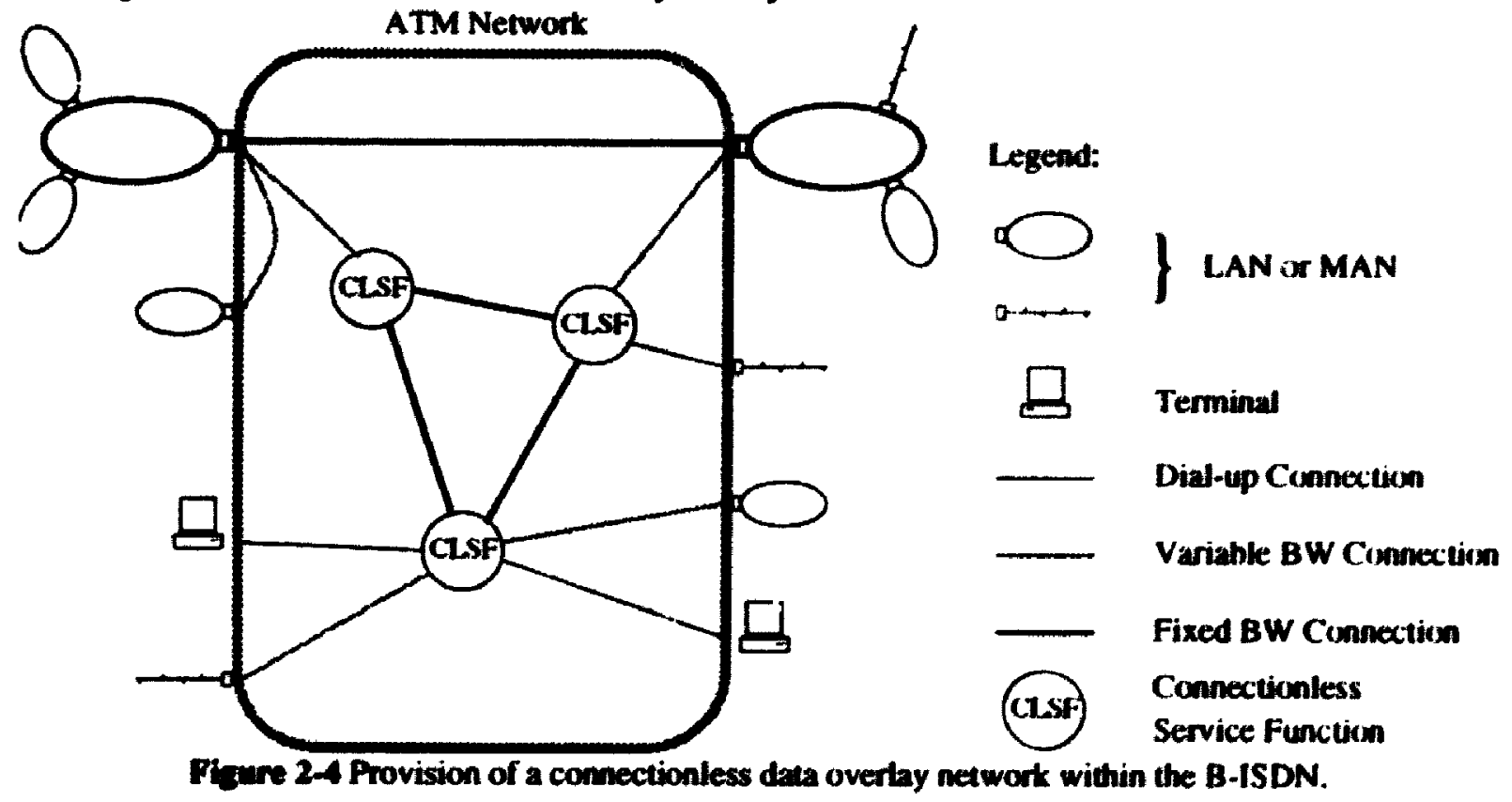


Given that the hybrid approach is a likely scenario for a future BISDN network, the CLSF should be designed with that environment in mind. Accordingly, it should be assumed as stated above that the CLSF will not handle high volume traffic, will have variable bandwidth connections to other entities, and will have the capability of receiving dial-up connections. This implies that the incoming and outgoing data rates, and the number of active connections at any one time, are dynamic quantities.

\subsection{Transporting Data Over ATM Networks.}

In order to transport data from a terminal. LAN or MAN via an ATM network, it must first be converted from the local transmission protocol into the ATM cell format. On reception, it must be converted to the new local format. These protocol conversions must be performed without loss of information or service degradation.

The ATM cell structure alone is not sufficient to ensure accurate transfer of data. A higher layer of protocol, the ATM Adaption Layer (AAL), is being defined by international standards bodies as part of the definition of B-ISDN. A number of different protocols are available at this level, the responsibility of each being to map a particular type of traffic into ATM cells. One version of this layer, known as Type 4 AAL, is specifically dedicated to carrying connectionless data traffic ${ }^{[9]}$.

Work within the CCITT and other standardisation bodies to date has centered on defining the actual protocols to be used within the ATM network. The ATM layer and AAL layer for data traffic has mostly been agreed upon. The current protocol definitions that apply to connectionless data are described in detail in the following subsection.

\subsubsection{Protocol Specifications.}

The BISDN protocol reference model as it pertains to the cunnectionless data service is given in Figure 2-5. The lowest layer of the model is the physical layer, which is respon- 
sible for the physical transmission of bits and uses either the SONET (USA. Japan. Australia) or ATM (Europe) transmission methods. Above that lies the ATM layer. which specifies how the data stream is broken up into 53 octet cells, including a 5 byte header. The header is used for routing of cells through the BISDN network. Both the ATM and physical layers are common to all BISDN services.

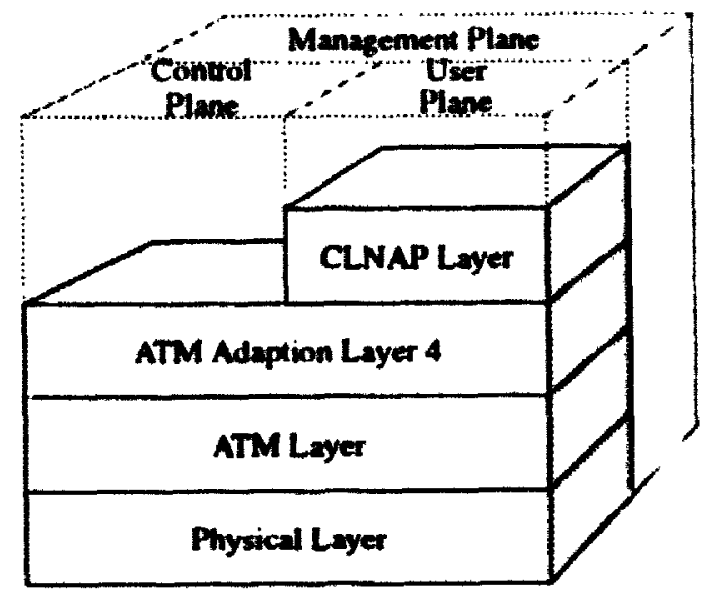

Pagure 2-5 BISDN Profocol Reference Model for Comnectionless Data.

Above the ATM layer lies the ATM Adaption Layer (AAL). Its function is to map the higher layer user data units into the ATM cell stream. The CCITT has defined the AAL for Type 4 (i.e. connectionless data) services in Recommendation $1.363^{[10]}$.

When CLSFs are being used to provide a direct connectionless service, then the services of an additional layer are required. This layer, the Connectionless Network Access Protocol (CLNAP) layer, lies within the user plane and carries network routing information. The CLNAP layer is partially defined in Draft Recommendation I.cis ${ }^{\text {[52]. }}$

The ATM adaption layer is divided up into two parts, the Segmentation and Reassembly (SAR) sublayer and the Convergence (CS) sublayer. The SAR sublayer sits directly on top of the ATM layer, and is responsible primarily for segmenting messages up into ATM cells. The CS sublayer is further divided into two parts - the Common Part Convergence Sublayer (CPCS) which lies directly on top of the SAR layer, and the Service Specific Convergence Sublayer (SSCS). The SAR and CPCS layers for both Type 3 (connection 
oriented data) and Type 4 AALs are the same. When a CLNAP layer is being used the SSCS layer is empty ${ }^{\lfloor 52 !}$.

Figure 2-6 illustrates the protocol stack for LAN-to-LAN communication via BISDN using CLSFs.

\begin{tabular}{|c|c|c|c|c|c|c|}
\hline \multicolumn{2}{|c|}{ Remote Bridge } & & \multicolumn{2}{|l|}{ CLSF } & \multicolumn{2}{|c|}{ Remove Bridge } \\
\hline \multirow{3}{*}{$\operatorname{Mnc}$} & CZNAP & \multirow{3}{*}{$\begin{array}{l}\text { Muliplex iD } \\
\text { vprva }\end{array}$} & \multirow{2}{*}{$\frac{\text { CLNAP }}{\text { and }}$} & \multirow{3}{*}{$\frac{\text { Mulpipled iD }}{\text { vpurva }}$} & CLNAP & \multirow{3}{*}{ MAC } \\
\hline & $\underset{\mathrm{SAR}}{\mathrm{MPS}}$ & & & & Ad & \\
\hline & ATM & & ATM & & ATM & \\
\hline PHYS & Phys & & Phn & & Fus & PHYS \\
\hline
\end{tabular}

Fyare 2-6 LAN-10-LAN Protocol Sexct for the Direct Connectionless Service.

\subsubsection{ATM Layer.}

The ATM layer is fundamental to the operation of the BISDN, and is common to all types of traffic. The basic unit of the ATM layer protocol is the cell, which consists of a 48 octet payload with a 5 octet header attached. The header uniquely defines which virtual circuit connection the cell belongs to. by means of the Virtual Path Identifier (VPI) and Virtual Circuit Identifier (VCI) fields. The format of the ATM cell at the boundaries of the CLSF, as currently defined by the CCTTT ${ }^{[52]}$, is as shown in Figure 2-7.

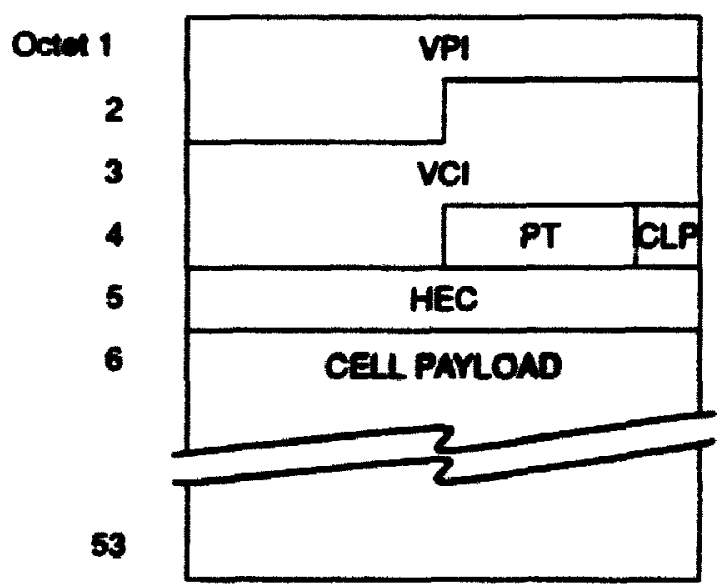

Digne 2-7 Forman of ATM Cell. 
Chapter 2: The Broadhand ISDN Connectionless Data Service.

Readers who wish to know more about the format and meaning of the various fields can consult the relevant standard. Recommendation $1.361^{[52]}$.

\subsubsection{AAL Layer}

The Adaption Layer for Type 4 service is detined by the CCITT in Recommendation $1.363^{[52]}$. As described above, the AAL is divided up into two sublayers for connectionless data operation, the Common Part Convergence Sublayer (CPCS) and the Segmentation and Reassembly (SAR) sublayer. The description of the AAL given in the recommendation specifies how the AAL interacts with the layer above it.

Provision is made for two modes of service, message mode and streaming mode. The distinction between the two is based on how the higher layer data unit (CLNAP packet) is mapped into the convergence sublayer data unit (CPCS-PDU). When operating in message mode, each higher layer unit is placed whole into a single CPCS-PDU. The entire CLNAP packet is transferred between the AAL and the CLNAP layers at once. before the CPCS-PDU is created or after it is received.

In streaming mode, the CLNAP packet is transferred between the layers piece by piece, where the transferral of each piece can be separated in time. An option known as internal pipelining allows for the CPCS-PDU to begin transmission before the entire CLNAP packet has been received from the higher layer. With this option, the CLNAP packet can be transmitted cell by cell as it is created in the sending entity. An abort service is included to enable partially transferred packets to be discarded.

In both cases, a single CLNAP packet is mapped into a single CPCS-PDU.

According to Draft Recommendation I.cls, when using the CLNAP for the direct BISDN connectionless service, either message or streaming mode may be used ${ }^{(52)}$. 
Chapter 2: The Broadband ISDN Connectionless Data Service.

The Type 4 AAL may only provide non-assured operation. That means that lost or corrupted CLNAP packets are not corrected by retransmission, although an option allows for the transfer of errored packets to the higher layers.

Multiplexing of cells at the AAL layer is provided. This means that within a given ATM connection (i.e. virtual circuit) there may be multiple AAL layer data connections. Thus more than one pair of end users may be communicating over a given virtual circuit at any one time. The multiplexing function is provided for within the SAR sublayer.

\subsubsection{SAR Sublayer.}

The SAR sublayer takes the yariable length CPCS-PDUs and breaks them into 44 $\alpha$. et SAR-PDUs for insertion into cell payloads at the ATM layer. In doing this it uses 4 of the octets to support its own functions, two of these in the header and two in the trailer.

The Type 4 SAR sublayer is defined by the CCrTT to have three main functions ${ }^{[10]}$. The first of these is preserving the integrity of the CPCS-PDU, which it does by inserting a 2 bit segment type (ST) field in the SAR-PDU header and a 6 bit length indicator (LI) in the trailer. The segment type indicator tells whether the cell is the first (BOM). middle (COM), last (EOM) or only (SSM) cell of this particular CPCS-PDU. The length indicator specifies to what extent this cell is filled by the CPCS-PDU, and so is used to determine the exact end of the higher layer data.

The second function is to detect errors. Bit errors are detected by a 10 bit cyclic redundancy check (CRC) in the trailer, and cell sequence errors (i.e. dropped or missing cells) are detected by a 4 bit sequence number (SN) in the header.

The third function is to provide for multiplexing and identifying the CPCS-PDUs on a single VC. by supporting SAR layer connections. This is done by means of a 9 bit Multiplex ID field in the header, where messages on each connection are assigned a unique (on that VC) connection identification number. Provision is also made for two priority levels 
of connection, by use of a priority (P) bit. This bit effectively extends the MII) tield to 10 bits. Not all of these potential connections need be supported at once in a particular application. How the connections are established and maintained has not yet been decided by the international standards bodies.

The format of the SAR-PDU for AAL type $4^{|52|}$ is given in Figure 2-8.

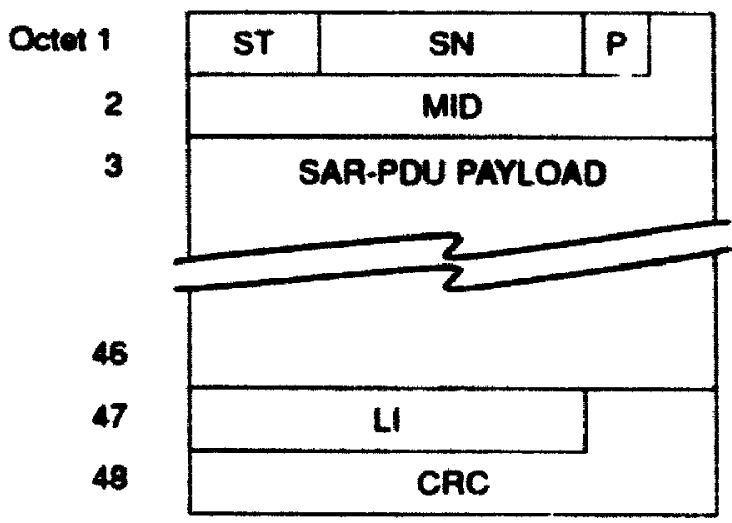

Figure 2-8 SAR-PDU Furmat.

Provision is also made for aborting the transmission of the CPCS-PDU, by the generation of an ABORT cell. The ABORT cell uses the same format as the other cell types, but has a ST value of EOM, a payload of all zeros and a LI field of all ones.

\subsubsection{CPCS Sublayer.}

The CPCS sublayer is responsible for preserving the integrity of the higher layer data unit. It provides error detection and handling procedures, as well as supporting either message or steaming modes as defined in Section 2.3.1.1.

The CPCS-PDU has a 4 octet header, variable length user data, a padding field and a 4 octet trailer. The header contains first a 1 octet Common Part Indicator (CPI) field, which is used to identify different types of CPCS-PDU by defining the meaning of the other fields as well as distinguishing data from signalling or maintenance frames. 
Chapter 2: The Broadhand ISDN Connectionless Data Service.

Following that is a one octet Beginning tag (B tag) which matches the End tag (E tag) field in the trailer. These fields provide an association between the first cell of the frame and the last, so that frames aren't accidentally concatenated in the event of cell loss.

The last field in the huader is the two octet Buffer Allocation size (BA size) field. which indicates the length of the entire CPCS-PDU in octets and is used to reserve the correct amount of buffer space in the receiver to accommodate this frame. It also acts as a pointer to the CS-PDU trailer, making it possible to detect strings of lost or inserted cells that weren't detected by the SAR sublayer.

The PAD tield is used to pad out the user data so that it fills out to a multiple of 4 octets, so that the following trailer is aligned on a 4 octet boundary for ease of VLSI or software implementation.

The trailer contains a 1 octet Alignment (AL) field, which fills out the unused octet in the trailer and does not convey any information. This is followed by the $\mathbf{E}$ tag, and a two octet Length indicator (Length) giving the total length of the higher layer data unit (CLNAP-PDU) in octets. This enables identification of the length of the padding field, and provides additional security against lost or gained cells.

The format of the CPCS-PDU is illustrated in Figure 2-9.

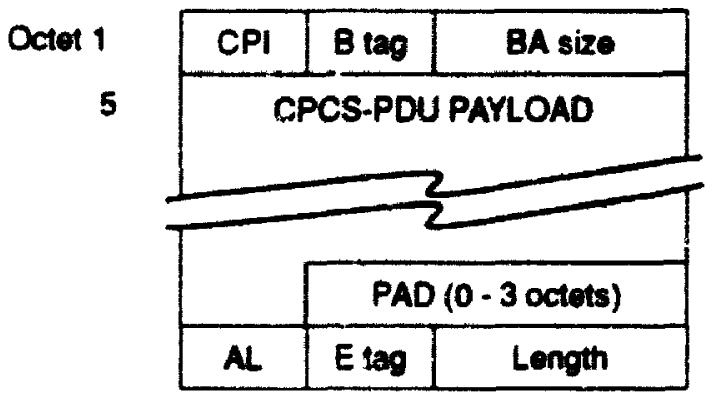

Figure 2-9 Format of the CPCS-PDU 


\subsubsection{CLNAP Layer.}

The CLNAP layer functionality and protocol are in the process of being detined by the CCITT in Draft Recommendation 1.cls ${ }^{[52]}$. The purpose of the CLNAP in the words of the CCITT is to "provide for the transparent transfer of variable size data units from a source to one or more destinations ... using a connectionless rechnique, including embedding destination and source addresses into each data unit." The protocol to be used by the CLNAP has been aligned with the ISO/EC CD $8802-6$ standard. which is the protocol used by SMDS. This has been done so as to simplify the interworking between existing equipment and the future BISDN.

The CLNAP layer is required to support the "Broadband Connectionless Data Bearer Service" described in Draft Recommendation F.812 $2^{|5|}$. These functions include preservation of higher layer data. routing of messages based on an E.164 address, source address validation, support of multicasting using group addresses, network charging, selection of a preferred carrier, and selection of $\downarrow$ desired quality of service. To provide for these functions, a variable length header and a trailer containing CLNAP protocol information are added to the user data.

The first four 32 bit words of the header contain the destination and source E.164 addresses. Each of these consists of the 60 bit address plus a 4 bit identifier which indicates whether the address is an individual or group address.

The fifth word contains five fields that support the additional functions of the CLNAP layer. The first of these is a 6 bit Higher Layer Protocol Identifier (HLPI) tield, which identifies what format the user information is in. It could be used for example to distinguish between Ethernet and Token Ring frames. This field is carried transparertly from end to end by the BISDN network. 
The next field is a 2 bit Pad Length field, which is used to indicate the length of a PAI field at the end of the frame. The PAD field is there to align the CLNAP trailer on a 4 octet boundary.

This is followed by a 4 bit QOS field, whicn indicates the quality of service requested for the packet. The specifications of a given quality of service have not been decided upon, but possible characteristics include packet delay and loss priority. The CCITT specifies a format for this field in ${ }^{|53|}$, but indicates that it is not yet agreed upon. The proposed format specifies that the QOS field be broken into a 3 bit delay subfield and a I bit loss subfield.

Following the QOS field is a 1 bit CRC indicator bit (CIB) which indicates the presence of an optional Cyclic Redundancy Check (CRC) in the trailer. The next field is a 3 bit Header Extension Length (HEL) field. This field indicates how many 32 bit words are contained in the ileader Extension (HE), which is an optional addition to the header. The Header Extension can be used to carry optional information used for such things as carrier selection.

The format of the CLNAP-PDU is given in Figure 2-10.

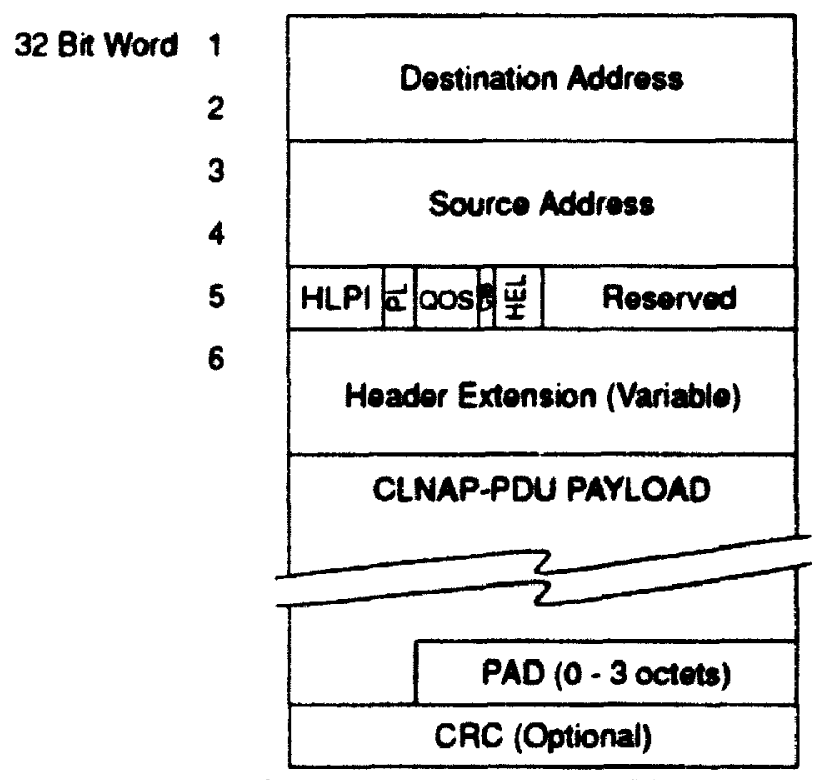

Pigure 2-10 Format of the CLNAP-PDU. 


\subsection{Summary of CLSF requirements.}

This section summarises the information given in this chapter that relates directly to the connectionless server. The required functionality and choices of options that will he used by the server architecture are presented is follows.

- The CLSF will be part of a connectionless virtual overlay network built on top of the ATM network. This overlay will consist of a number of users and connectionless servers joined by virtual path or virtual circuit ATM connections.

- The CLSF will route data around the network based on the destinition addresses carried in data packets. It does this by translating the virtual circuit identifiers, allowing the ATM network to switch the data.

- The connections that make up the overlay network will all be variable bandwidth. This means that control procedures will enable the network entities to adjust the agreed bandwidth parameters of the virtual connections in response to observed traffic levels.

- It will be possible for terminals to access the CLSF through the use of ATM connection establishment procedures, in other words to "dial up" the CLSF at the beginning of a runnectionless service session.

- The CLSF must comply with the current CCITT standards that apply to the BISDN, in particular those specifying the data formats and functionality required of the ATM. AAL and CLNAP layers. These requirements are as follows.

- At the ATM layer, the CLSF terminates virtual circuit and virtual path connections. It must not issue cells on the outgoing connections that violate the negotiated bandwidth parameters.

- At the AAL layer, the CLSF must support an additional level of multiplexing using SAR sublayer connections. AAL layer connections are terminated at the CL.SF. The 
method by which such connections can be established and cleared has not been established, but will involve control plane procedures.

- AAL streaming mode with the pipelining option will be used with this CLSF architecture, so that incoming packets can be processed and begin retransmission before they have been fully received. This means that abort procedures will be supported, but the delivery of errored packets to the user will not. Errored packets will instead be discarded, and will cause the initiation of abort procedures on the outgoing connection.

- At the CLNAP layer, the CLSF will provide the broadband connectionless data bearer service described in Draft Recommendation F.812. This requires that the following capabilities are supported:

- Source address validation,

- E.164 addressing.

- multicasting using group addresses.

- capacity for network charging.

- interworking with other data services (carrier selection),

- support of quality of service selection. 


\section{Chapter 3: Connectionless Server Architecture.}

\subsection{Initial Design Approach.}

This section describes the initial attempts at producing a connectionless server architecture capable of providing the direct BISDN connectionless service described in the previous chapter. The two preliminary approaches presented in this section introduce the principles of operation used in the final design, as well as illustrating some of the problems that needed to be overcome.

\subsubsection{Single Processor system.}

The first approach used was the single processor architecture illustrated in Figure 3-1. The system has a single input physical link and an output link. Each link has terminating hardware which is responsible for mapping the ATM cell stream into and out of the buffer memory. The processor carries out the translation between incoming virtual circuits and outgoing ones.

Incoming cells pass through the physical interface, and enter serially into the incoming cell module. This device routes the first seven octets of active cells (containing the ATM and SAR headers) into the processing queue, then stores the rest of the cell as it comes in into the cell buffer memory. The CLNAP header in the first cell of each message is also sent to the queue. The module also performs a CRC check on the data as it comes in. so that errored cells may be discarded.

The Message Processor examines each entry in the processing queue and performs the routing algorithm on each beginning of message (BOM) and single segment message (SSM) cell. An entry for that message is placed in the Active Message Table for each 


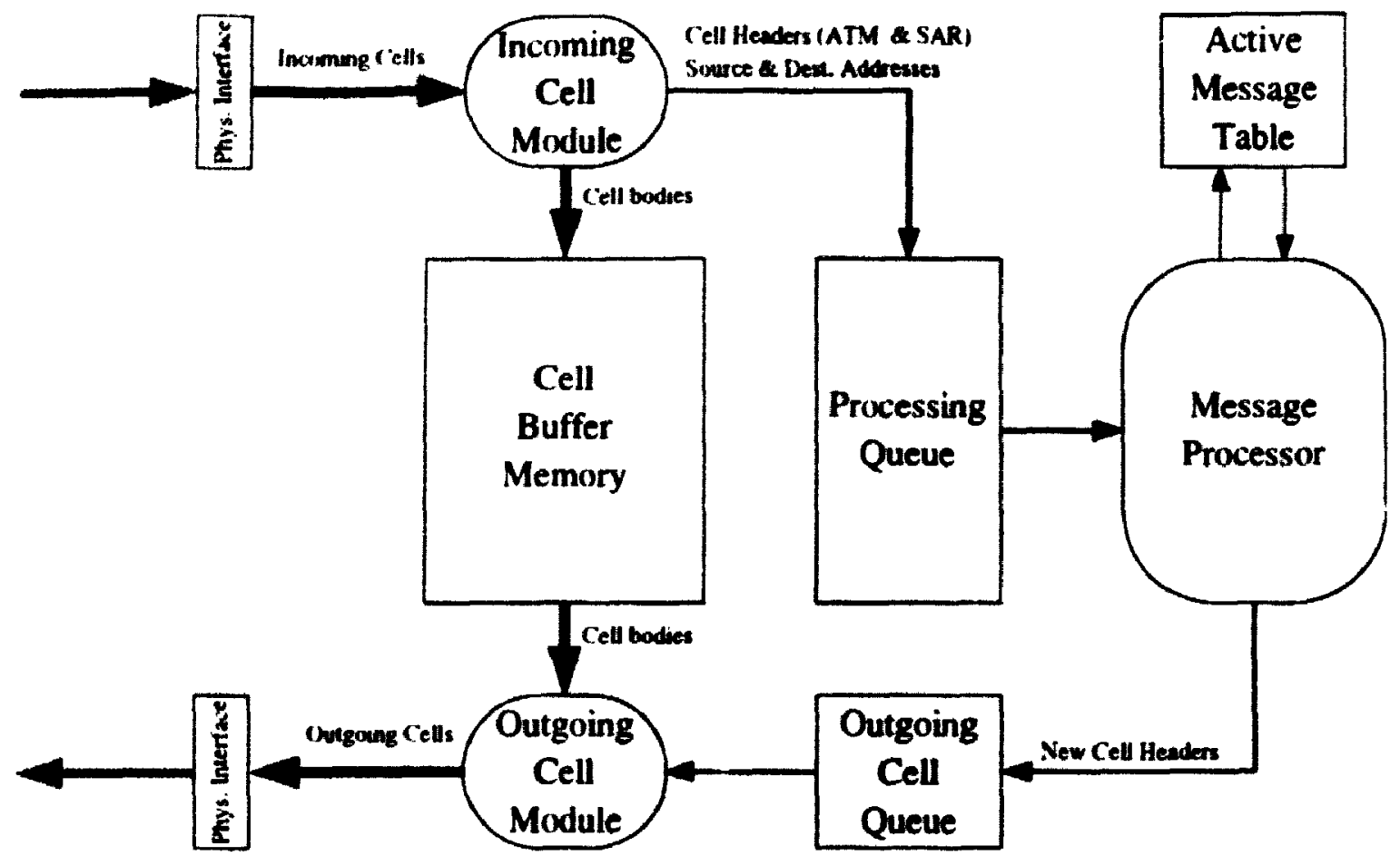

Figure 3-1 Initial Design: Single Processor Basic Architecture.

BOM cell, containing the destination virtual circuit identifier ( $\mathrm{VCl}$ ) for the message. Continuation of message (COM) cells cause a lookup in the active message table, and end of message (EOM) cells cause the entry to be removed. For each cell, a new header containing the new $\mathrm{VCl}$ is generated and placed in the outgoing cell queue.

The outgoing cell module takes cell headers from the outgoing cell queue and retrieves the cell bodies from the cell buffer memory. New CRC fields are generated, and the reassembled cell is placed on the outgoing physical link.

In addition the message processor is also responsible for monitoring the status of its portion of the connectionless overlay network, carrying out VC bandwidth control algorithms, communicating with other CLSFs and with the underlying ATM network, and other managerial tasks.

A major problem with this single processor approach is speed. In order to achieve a satisfactory delay, throughput and link utilisation, it is necessary to have the average processing time per cell to be of the order of the physical transmission time. For a 155 Mbps 
Chapter 3: Connectionless Server Architocture.

physical link, that time is $2.74 \mu \mathrm{s}$ (53 octets at $51.7 \mathrm{~ns} /$ octet). For a 622 Mhps physical link, the processing time must be of the order of $682 \mathrm{~ns}$ per cell. Using a $30 \mathrm{Mhz}$ RISC processor, that translates to 82 instructions at $155 \mathrm{Mbps}$ and 20 instructions it 622 Mhps. With a single processor responsible for carrying out all the reassembly, routing, transmission and maintenance procedures in this approach. the processing time limits simply can not be maintained. The amount of processing overhead involved even in the handling of a COM cell means that this single processor per link approach is not suitable.

\subsubsection{Multi-Processor system.}

The next attempt was to try the multi-processor approach illustrated in Figure 3-2. This system uses a number of processors operating in parallel on a smaller number of physical links. A system manager takes care of overhead functions such as traftic monitoring and network maintenance, leaving the other processors free to work exclusively with the data traffic. The advantage of this approach is that more processing power is available per connection, as well as an increased system capacity due to the increased number of input and output links.

Each incoming and outgouig physical link has its own cell module, which is responsible for assembling and disassembling cells. The bodies are placed in a co.nmon buffer memory, accessible to all links, and the headers into the processing queue. Lells that have errors are reported to the system manager, as well as cells containing inter-CLSF communications and control signals from the ATM network.

The processors all have access to the processing queue. When a processor becomes free, it removes an entry from the queue and acts upon it in the same way as for the single processor case described above.

Once a new $\mathrm{VCl}$ for a cell has been decided on, the processor selects which outgoing link to send the cell on by selecting the appropriate outgoing cell queue. The outgoing cell 


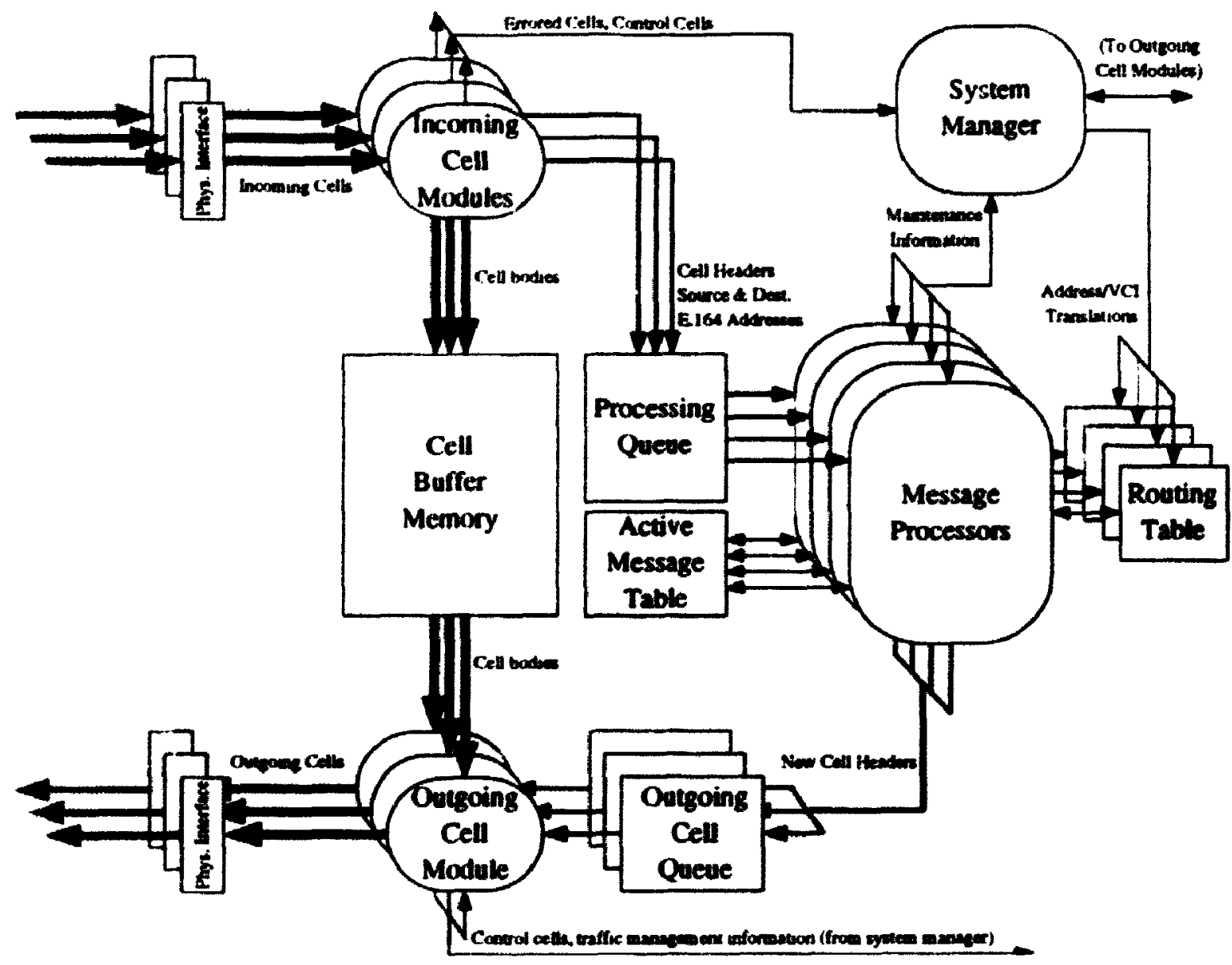

Phoure 3-2 Initial Design: Multi Processor Basic Architecture

module associated with that queue then retrieves the body from the common memory and places it on the physical link.

Changes in network topology are handled by dynamic maintenance of the routing table, which contains the E.164 address/VCI translation table used by the message processors. This table is common to all processors, but because of the amount of accessing that is requirec of it each processor will have its own local copy. This table will also contain a list of authorised source addresses and VCIs for address validation, and other information necessary to carry out the security features of the connectionless service.

With this design the cell buffer memory is common to all links, enabling data that arrives on one particular link to depart on another. However, this poses problems with memory access timing, as well as the difficulty of finding a sufficiently large and fast 
multi-port memory. This can be solved by each incoming/outgoing link pair having its own buffer. Cells can not then switch between physical links, but the outgoing ATM connections could be set up as many-to-one connections from all the outputs. This problem is also faced when trying to increase the capacity of the final design. and this particular solution is elaborated upon further in Chapter Five.

The processing queue must be common in this approach, so as to enable any processor to access cells from any link. As cell headers are taken from the queue only once per cell time per link, this does not result in memory access problems. Care must be taken. though. with the routing of data to the processors.

The active message table is shown here as being common, so that each processor can recognise COM and EOM cells belonging to messages whose BOM cell was seen by another processor. Two problems arise from this approach, the tirst being memory access speed again. The second problem arises because BOM cells take longer to process than COM cells. It is quite possible that one processor would be working on a COM cell before the active message table entry had been created by the processor that received the BOM cell of the same message, resulting in the COM processor being held up for a significant amount of time. Similar timing mismatches can occur with any two cells from a single message, resulting in extreme difficulty maintaining cell sequence integrity.

The other problem with this approach once again is speed. The amount of processing needed per cell is still very high, and so the number of processors would need to be quite a lot higher than the number of links, particularly at link rates of $622 \mathrm{Mbps}$. The approach taken in the final design to solve this problem involves the use of customised hardware and pipelining of operation, as the rest of this chapter describes. 


\subsection{Functional Overview.}

After reviewing the problems encountered with the original designs, the following system is proposed as a solution to the design objectives. This section describes the overall operation of the system as a whole. The following sections expand on the details of the system building blocks. Readers may find it easier to become well acquainted with the principles of operation described in this section, before tackling the detailed descriptions in the remainder of the chapter.

The functional architecture of this design is illustrated in Figure 3-3. The system consists of a number of functional 'tocks with some additional supporting circuitry, and is designed to operate on a single physical link. Each block performs a subset of the processing required on the cells, resulting in some pipelining of operation.

The basic principle of operation of this system is quite simple. Incoming cells are stripped of their headers and the bodies stored in a cell RAM, while the headers are stored in a header RAM in groups that represent the packets. Each packet is examined for its destination address and assigned an outgoing virtual circuit identifier. The cell headers of the packets are read from the header RAM at a rate corresponding to the allocated bandwidth of the outgoing virtual circuit, and placed in an outgoing queue. The cell bodies are then fetched from the cell RAM and the reconstructed cells are transmitted on the outgoing link. The difference between this and the earlier approaches is in the way in which the header processing is performed.

The Incoming Cell Unit is responsible for separating incoming cell header information from the cell bodies, and checking the incoming cells for bit errors. The cell RAM, which is effectively a large buffer, holds the bodies until they are ready to be retransmitted, while the headers are placed in the Incoming Header Queve. Once processing is complete, the Outgoing Cell Unit retrieves the cell bodies from the RAM and inserts new headers from the Outgoing Header Queue, adds new CRC checks, and transmits them on the outgoing 
Chapter 3: Comnectionless Server Architecture.

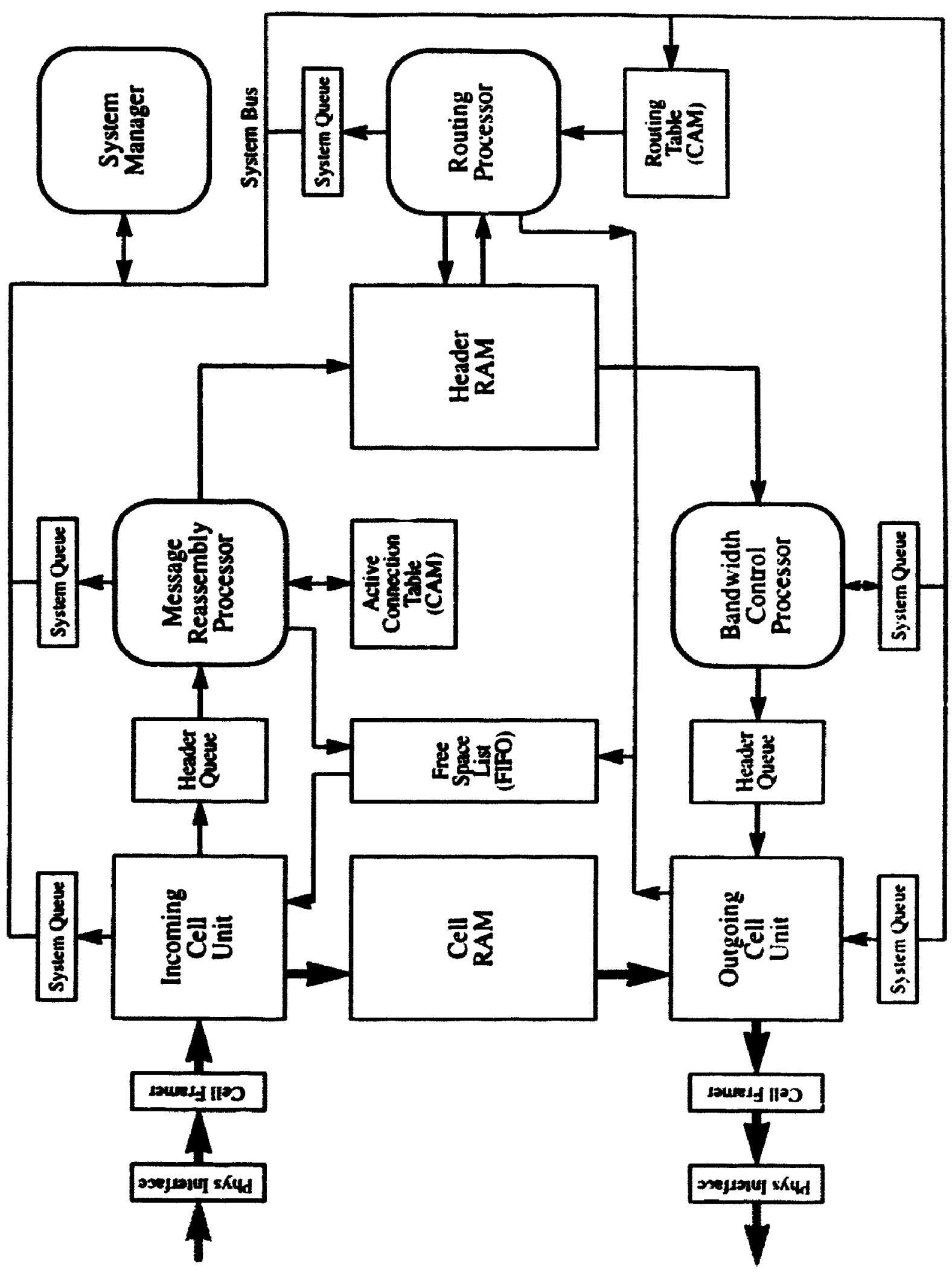

Figure 3-3 Functional Architecture of the CLSF. 
links. Both the ICU and $\mathrm{OCU}$ are simple hardware devices (finite state machines) with no processor control.

The Cell RAM contents are maintained through use of the Free Space List. This list is simply a list of the start lociations of all unused cell storage areas in the RAM, stored in a FIFO buffer. When the ICU places a cell in the RAM, it uses the address from the head of the buffer. The address becomes the cell's identifier within the system, and is kept with the header information. When the OCU transmits a cell, it returns the address to the bottom of the list. Other units may also place a cell's address in the list when errors occur, effectively discarding the cell from the system.

The cell headers, which include the ATM, AAL and CLNAP layer headers, are processed first by the Message Reassembly Processor. This unit sorts the randomly interleaved incoming cell stream into packets or messages (CLNAP-PDUs), and stores these messages (cell headers only) in the Header RAM. It also detects errors involving lost or misinserted cells. The unit is based on a single RISC processor, and operates at cell speeds with the aid of a lookup table based on Content Addressable Memory.

Once a message has been placed in the Header RAM, the Routing Processor retrieves its source and destination addresses, and makes a routing decision based on the information stored in its Routing Table. The message is added to a logical output queue within the Header RAM that corresponds to its new outgoing virtual circuit. This processor is also responsible for implementing the CLNAP-layer functions, such as source address validation. yuality of service support, network charging, etc. It too is based on a RISC processor, and must perform its complex procedures at message speeds.

The Bandwidth Control Processor takes the header information of the cells of each message in each output queue, and sends those headers to the $O C U$ at a rate corresponding to the allocated traffic parameters of the outgoing virtual circuit. It also monitors the amount of traffic on the outgoing link, and detects when a virtual circuit requires more or 
less than its allocated bandwidth. The unit is also processor based. and although it operates at cell speeds it increases its service rate by taking the cells in blocks.

Overseeing the entire system is the System Manager, which carries out the control and maintenance plane functions. It monitors the state of the system. and notities the ATM control when the traffic parameters of incoming or outgoing links need to be adjusted. It also communicates with other CLSF entities, and helps control the connectionless overlay network. It receives and generates its messages through the system bus, which also allows it to communicate with the other entities in the system. It has direct access to the inconning and outgoing cell streams via the ICU and OCU.

The Bandwidth Control Processor keeps the System Manager informed of the traftic on the outgoing VC connections. Should it be necessary to increase a connection 's bandwidth, for example, the System Manager will generate a request for more bandwidth in the form of an ATM control cell which is sent to the OCU and transmitted immediately, bypassing the output queue.

Similarly, incoming control cells are detected by the ICU and forwarded directly to the System Manager, as for example when a terminal wishes to establish or clear a virtual circuit or SAR layer connection. The System Manager would receive the request, and would notify all the other system components of the change via the system bus.

\subsection{Physical Interfaces.}

The incoming cell stream, carried on optical fibre or another physical medium, is fed into the physical interface which converts it to the appropriate electrical signals. The serial data passes into the cell framer circuit, which performs the physical layer functions as defined in the CCITT recommendations. In countries where the SONET physical layer is used, this circuit would be a STS-3c (155 Mbits/s) or STS-12 (622 Mbits/s) framer circuit. STS-3c framers are already available in integrated circuit form ${ }^{|17|}$. The output of the 
framer curcuit is a cell stream in the form of a series of octets, carried on an 8 -bit bus. It may also output signals indicating the start of each cell.

After processing, the outgoing cell stream passes through a similar framing circuit which places the cell stream irito the appropriate physical layer format, and outputs them as electrical signals. The signals are converted at the physical interface into the appropriate medium for transmission on the outgoing link.

\subsection{Incoming Cell Unit.}

\subsubsection{Operation of the ICU.}

The formats of the different types of cells at the boundaries of the physical interface are illustrated in Figure 3-4. Refer to Section 2.3.1 on page 20 for an explanation of the meanings of the various tields.

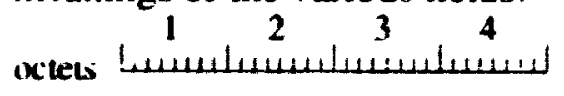

$\left.\begin{array}{l}1] \\ 2 \\ 3 \\ 4 \\ 4 \\ 5 \\ 5 \\ 6 \\ 7 \\ 7 \\ 8 \\ 4 \\ 9 \\ 11 \\ 11 \\ 12 \\ 12\end{array}\right]$

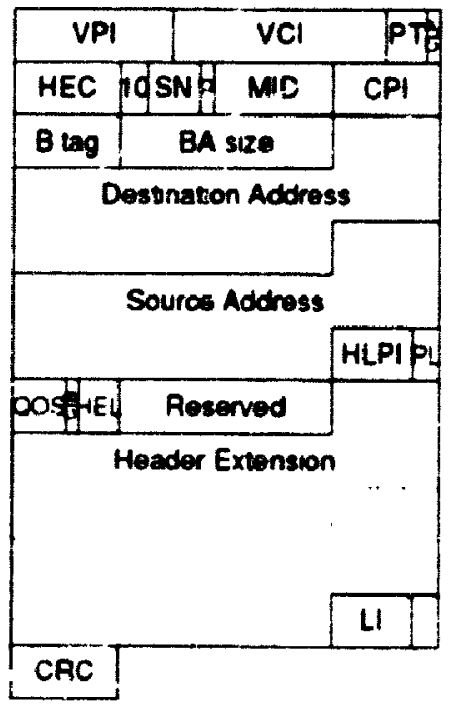

a) BOM cell.

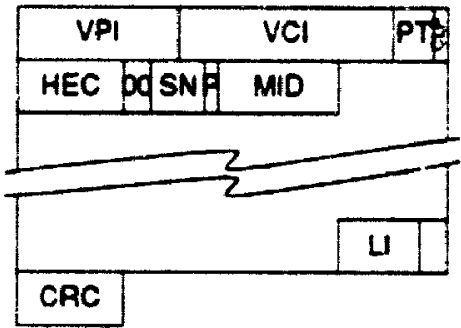

b) COM cell.

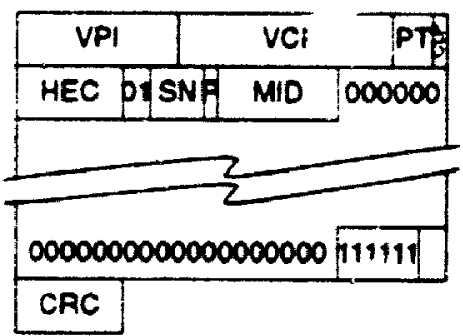

d) ABORT cell.

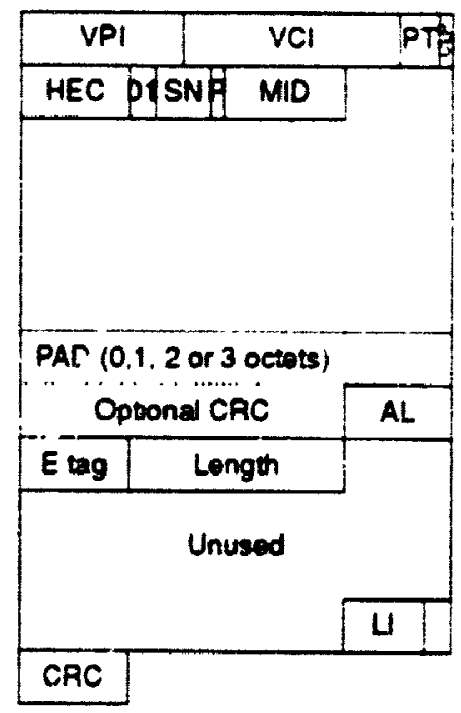

c) EOM cell.

Figure 34 Format of incoming cells 
The Incoming Cell L'nit takes the cells in this format. and extracts the protocol header information. The headers are placed in the Header Queue along with a cell identitier, while the cell bodies (the 44 octet SAR layer payload) are stored in the Cell RAM. Cells intended for the system manager are detected and are placed instead in the System Quecue. This process is described in detail in the following subsections.

\subsubsection{Functions common to all cells.}

Prior to a cell's arrival. the ICU requests a free memory block inddress from the Free Space List. This address indicates where the cell body will be stored in the cell RAM. and also serves as the cell's identifier while it is in the system. The memory address hecomes the first data word of the header queue entry for that cell.

The first four octets received from the framer circuit (Section 3.3) comprise the ATM header. This contains the VPI/VCI value, the Payload Type (PT) and the Cell Loss Priority (CLP) fields. The PT field is checked to determine if the cell is a maintenance (OAM) or resource management cell. It is possible for certain VPI/VCI values to indicate a system cell (such as for signalling connections, etc.). so these fields are also examined. A cyclic redundancy check is performed on the ATM header, using the HEC octet. The four ATM header octets become the second data word stored in the header queue, while the HEC octet is dropped.

The next two bytes to arrive are the SAR layer header. This consists of the Segment Type (ST), Sequence Number (SN), Priority (P) and Multiplex Identifier (MII)) fields. In the ICU, only the ST field is checked. The behavior of the ICU for the rest of the cell depends on the value of this field.

The SAR sublayer header and trailer information are placed together in the header queue as the next entry. Doing this has the effeci of aligning the remainder of the cell information on the appropriate 32-bit boundary. Thus, the first two bytes of this header word carry the SAR.PDU header and the last two the SAR-PDU trailer (LI and CKC 
fields). The rest of the data received in the cell (the SAR-PDU payload) is autornatically copied to the cell RAM.

\subsubsection{B()M cell procedures.}

If the cell is a BOM or SSM cell (ST value 10 or 11 ), then the next few words contain hearler information. The first four octets to arrive are the convergence sublayer header (CPCS-PDU header). This header is not used by the ICU, and passes into the header queue as a single 32-bit word. The variable length CLNAP layer header follows, and the first four 32-bit words in that header (the source and destination addresses) are passed into the header queue as they arrive.

The fifth CLNAP header word contains among other things the Header Extension Length (HEL) field, which indicates how many 32-bit words are in the Header Extension field! The HEL field is used by tik ICU to determine how many more cell words contain header information. The appropriate number are placed in the header entry.

The format of the BOM header queue entry is presented in Figure 3-5a.

\subsubsection{COM cell procedures.}

If the ST ticld indicates a COM cell (value 00) then the header queue entry is complete. The remainder of the cell goes into the cell RAN، as it is received. The COM header entry is illustrated in Figure 3-5b.

\subsubsection{EOM cell procedures.}

If the cell is an EOM or SSM cell then the convergence sublayer trailer must be extracted from the information stream. This is done by making use of the Length Indicator

1 The sire of the HEL field is such that up to 7 words can be included, however only four of these words will fit within the BOM cell. As a result. this application requres a maxumum HE length of 4 words. See Section 2.3.1.5 on page 27 fir detals of the HEL tield. 
Chapter 3: Connectiomless Server Architeclure.

\begin{tabular}{|c|c|c|c|}
\hline & \multicolumn{3}{|c|}{ Cell Memory Locaton } \\
\hline \multicolumn{2}{|c|}{ VPI } & VC & PT: \\
\hline IdSSH & MID & 4 & CRC \\
\hline CPI & $8 \operatorname{tag}$ & \multicolumn{2}{|c|}{ BA size } \\
\hline \multicolumn{4}{|c|}{ Destinabon Address } \\
\hline \multicolumn{4}{|c|}{ Source Address } \\
\hline HLPI & & & rved \\
\hline
\end{tabular}

Header Extension

a) BOM cell entry

\begin{tabular}{|c|c|c|c|}
\hline & \multicolumn{3}{|c|}{ Cell Memory Locaton } \\
\hline VPI & & $\mathrm{VCl}$ & PTE \\
\hline bd SNA & MID & 4 & CRC \\
\hline
\end{tabular}

b) COM cell entry

\begin{tabular}{|c|c|c|c|}
\hline & \multicolumn{3}{|c|}{ NuH Location } \\
\hline VPI & & VCI & PT \\
\hline PISNH & MID & 111111 & CRC \\
\hline
\end{tabular}

d) ABORT cell entry

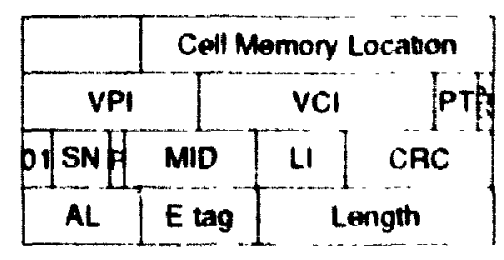

c) EOM cell entry

Figure 3-5 Format of Header Queue entries.

(LI) field in the cell trailer. The LI indicates the number of octets of user data contained in the SAR-PDU payload. Thus, the value of the LI points to the location of the last octet of the CPCS-PDU in the cell. By disregarding the two least significant bits of the III (dividing the LI by 4), a pointer to the last 32-bit word of the CPCS-PDU can be obtained. Because the PAD field aligns the trailer to a multiple of 32 bits, this word contains the entire CPCS-PDU trailer. The whole cell must be buffered in the ICU as it comes in, and the LI used to select which of the words goes to the header queue.

The format of the EOM cell entry is shown in Figure 3-5c.

\subsubsection{System cell and error handling procedures.}

If the VPIVCI is zero, indicating that the cell is idle or unassigned, then the header is discarded and the rest of the cell ignored. The same occurs if the check on the HEC field indicates an error in the ATM header.

If the VPIVCI field indicates a cell intended for the System Manager, the entire cell is routed to the system queue as it arrives. The cell is not copied to the cell RAM. 
A CRC check is performed on the cell payloads as they are received, using the CRC field in the SAR trailer. Errored cells are discarded, by dropping the header entries and reusing the cell RAM location for the next cell.

A LI greater than $\mathbf{4 4}$ indicates an error and the cell is discarded, unless the $\mathrm{LI}$ value is 111111 in which case the cell is an ABORT cell. An ABORT cell is stored in the header queue as an EOM cell without the CPCS-PDU trailer word. Rather than retain the cell contents, a dummy cell RAM location goes in the header queue and the current location is reused. The ABORT cell entry is illustrated in Figure 3-5d.

\subsubsection{Hardware Description.}

A functional diagram of the incoming cell unit is given in Figure 3-6. The unit is entirely a hardware device, possibly implemented in custom or semi-custom VLSI, that carries out the functions described in Section 3.4.1. The purposes of the various functional units are explained in the following paragraphs.

The basic principles of this device are as follows. Cells enter the ICU from the framer circuit on an 8 bit bus, one octet at a time. The octets are passed through the HEC and CRC checker circuits as they come in (which detect bit errors in the cell), then they are fod into a multiplexer which converts them into a 32 bit format. The multiplexer also performs the byte alignment functions required to format the header entry as in Figure 3-5 on page 43. The aligned words are placed one by one into the Primary Registers, and are checked as they come in to determine whether the cell is to go to the System or Header Queues or to be discarded. The words corresponding to the cell bodies are also placed into the Cell RAM, at locations specified by the address counter. Once the entire cell has been received. the contents of the Primary registers are transferred to the Secondary registers, and the headers are shifted into the appropriate queue while the next cell is being received.

The control of the ICU is divided butween two hardware units, the Input Control and the Transfer Control units. The Input Control unit is responsible for sending the incoming 
Chapter 3: Connectionless Server Architexture.

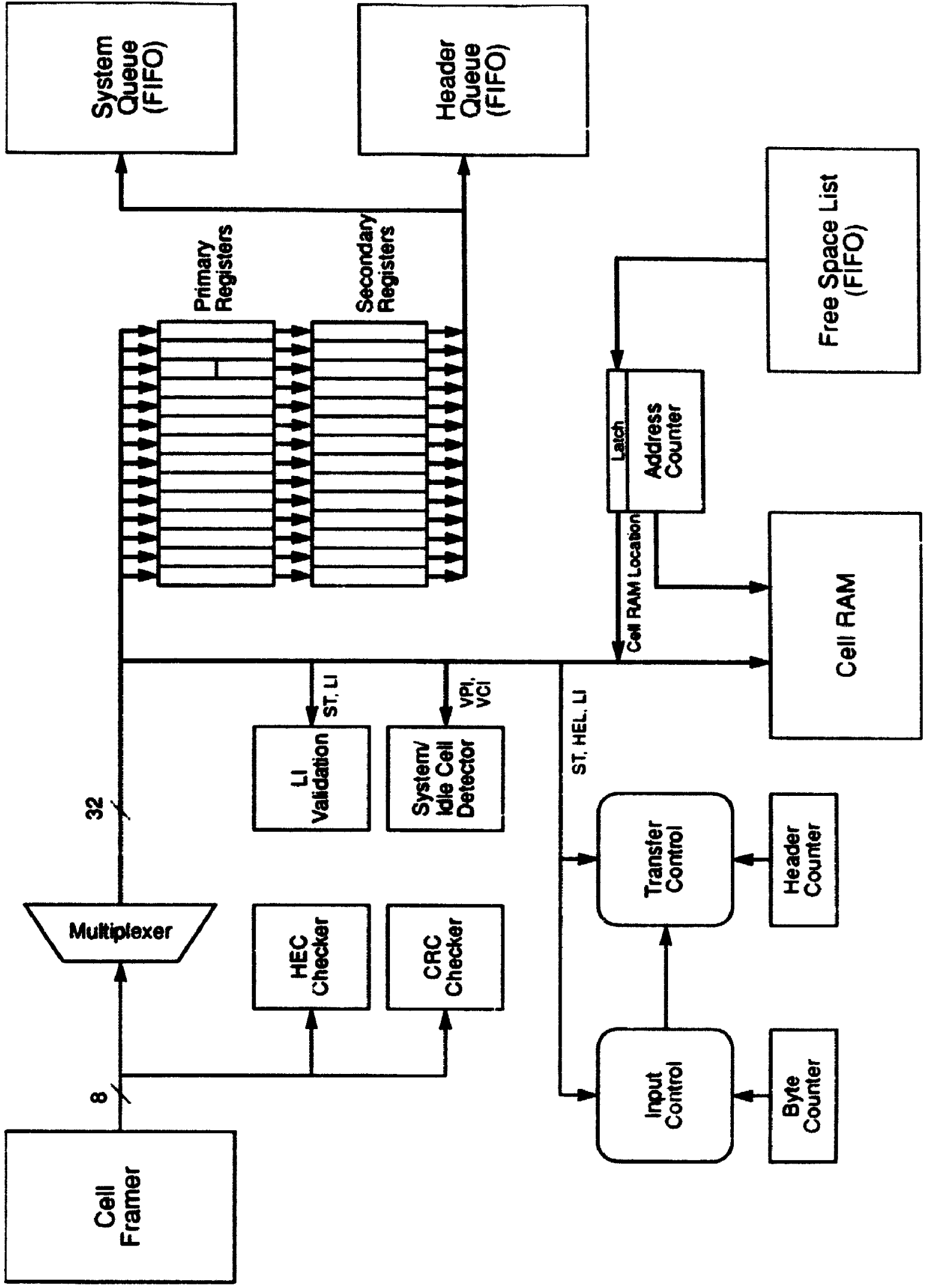

Figure 3-6 Functionad Architecture of ICU 
words to the appropriate places, and for detecting and responding to error conditions. The Transfer Control unit shifts the header data from the primary to the secondary registers, then ensures that the right number of words get sent to the correct queue. Thus, the transfer control must detect the cell type and construct an appropriate header queue entry.

Refer to Appendix A for a detailed description of the operation of this device.

\subsection{Cell RAM and Free Space List.}

The cell RAM is based on a 32 bit, dual port random access memory. The Incoming Cell unit has access to one side of the RAM, while the Outgning Cell unit has access to the other. Both of these units are working in synchronisation with their respective cell streams, so access to the RAM is in a deterministic manner. As a result, the RAM does not need to be truly dual port in nature, but can instead work on a time allocation principle, i.e. the ICU is allocated the first half of each word time slot, and the OCU the second half.

If the physical link is operating at $155.52 \mathrm{Mbps}$, then the time taken to transmit or receive an entire $\mathbf{3 2}$ bit word is $\mathbf{2 0 6} \mathrm{ns}$ and so the memory must have a cycle time less than 102 ns, easily achievable with current technology. For physical links operating at 622.08 Mbps, the word time is $51 \mathrm{~ns}$ and so the memory must have an access time less than $25 \mathrm{~ns}$ or be truly dual port in nature.

The data in the memory is organised in blocks of $\mathbf{4 4}$ words, corresponding to cell payloads. The addresses of the first word of each block are stored in the free space list FIFO memory. The address space of the memory is 3 octets or 24 bits, giving a maximum mem-

ory size of $16.7 \times 10^{6}$ words or $1.52 \times 10^{6}$ cells, corresponding to about 4.3 seconds of transmission with a fully loaded $155 \mathrm{Mbps}$ interface or 0.97 seconds at a $622 \mathrm{Mbps}$ interface.

Built in to the Free Space List FIFO is some control circuitry which is capable of detecting when the number of entries in the list (i.e. the number of unused locations in the 
Chapter 3: Connectionless Server An'hulecture.

RAM) gets too low. This causes a signal to be sent to the System Manager. allowing it to instigate congestion control procedures before cells have to be dropped.

At system initialisation time the Free Space List must be primed, by filling it with the addresses of all the cell locations in the RAM. This is done by the initialisation routines of either the Message Reassembly or Routing processors.

\subsection{Message Reassembly Processor and Active Connection Table.}

The message reassembly processor is responsible for sorting the cells as they come in into message areas in the Header RAM. It effectively carries out the reassembly function of the SAR sublayer. It also implements the CPCS sublayer functionc. The procedures are based on those described in Appendix 2 of CCITT Recommendation $1.3633^{1521}$.

Basically, the MRP takes the incoming cell stream which consists of cells from a num ber of sources multiplexed together, and reassembles those cells back into CLNAP layer messages. It does this by examining the AAL layer information stored in the cell headers which have been placed in the incoming header queue. The cell ID numbers (cell RAM locations) of all the cells of a particular message are placed together in the Header RAM. The CLNAP layer information for a particular message is stored in a queue in the Header RAM corresponding to the desired quality of service.

The MRP uses the Active Connection table to keep track of the reassembly processes for each connection. This table is stored partly in conventional RAM, and partly in content addressable memory. To understand the operation of the MRP, it helps to first review what information is kept in this table. 


\subsubsection{Active Connection Table format.}

For each active data connection, the MRP maintains an entry in the Active Connection table. The entry consists of the connection identifier (made up of the VPI, VCI, P and MID fields), and the state of the receiver processes for that connection, as defined in Recommendation 1.363 . The state of the SAR receiver process is indicated by two state variables, the machine state sar_S which can take the values IDLE $(=0)$ or REASS $(=1)$, and the expected sequence number rev_SN which is incremented modulo 16. The state of the CPCS receiver process is stored using three state variables, the machine state epes_S which takes the values IDLE $(=00)$, REASS $(=10)$ or $A B O R T(=01$ or 11$)$, the current beginning/end tag value rev_BEtag, and the expected CS-PDU size rev_BASize. It also stores the number of octets received so far in this packet, in the variable rev_size. Last but not least. the location in Header RAM of the current message is kept in this table.

The Active Connection table is stored partly in a 64 bit Content Addressable Memory, and partly in conventional RAM. The CAM stores the connection identifier fields and the sequence number field in the same locations as they appear in the header queue entries, so that they may be quickly matched against the incoming headers. The rest of the CAM is taken up with a pointer into conventional RAM, corresponding to the location of the rest of the entries. The format of the AC table is given in Figure 3-7.
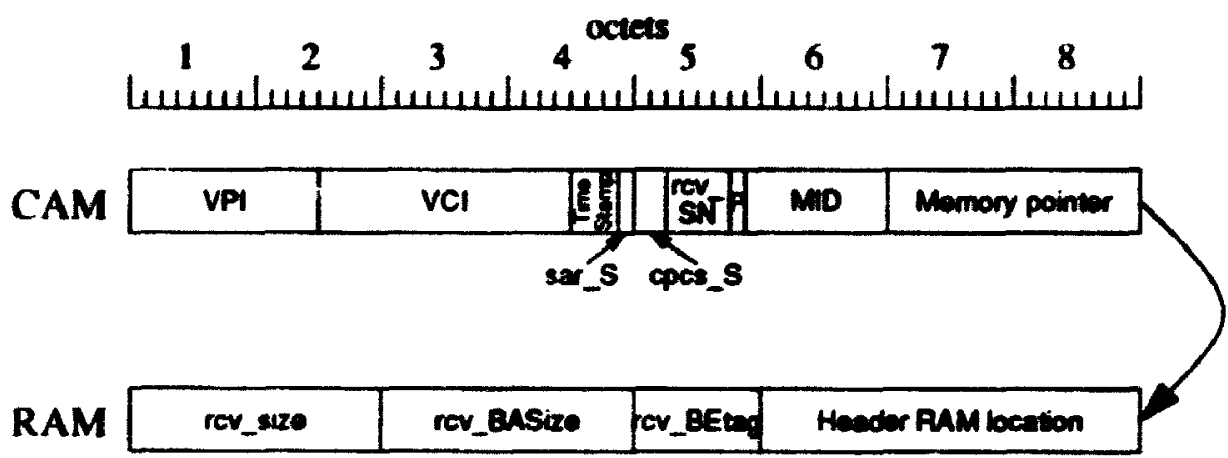

Figare 3-7 Format of Active Connection Table entries 


\subsubsection{MRP Operation.}

The action that the MRP takes when a cell arrives depends on the value of the ST tield. i.e. whether the cell is a BOM. COM. EOM, or SSM cell. For every cell, the tirst step will be a search of the CAM part of the active message table, to determine which message connection the cell belongs to.

A CAM search is made by masking out with donitcures the fields that we are not interested in, and instructing the CAM to match the unmasked part of the input dgainst all the data words in its memory ${ }^{[12]}$. If a match is found, the CAM will output the entire value of the matching data word. Assuming that the AC table is * c up correctly, then at most one match will be found, corresponding to that cell's cor ction identifiers. The mask word used by the MRP to find a match is illustrated in Figu : 3-8.

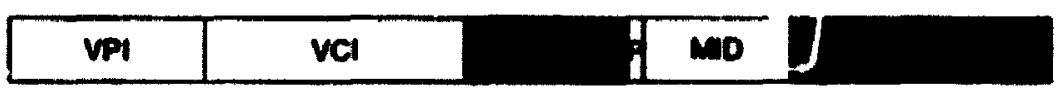

Figure 3-8 CAM mask used to identify messages.

If a special purpose CAM is used, the address of the matching word could be output as well as its contents. This address could then correspond to the location in RAM of the additional information, and the Memory Pointer fielt is not required. Which of these alternatives is used depends on the availability of suitable CAM devices.

The MRP places the cell header information into the Header RAM. The Header RAM is arranged as a number of linked lists, with each node in the lists being a block of memory locations. One of the linked lists in the memory, the free block list. contains all of the unused blocks in the Header RAM. When the MRP gets a free block, it removes the entry from the free block list.

The Header RAM also contains a number of message queves, each corresponding to a different quality of service. Each message queue is stored as another linked list, with the 
blocks containing message entries. A message entry will contain a pointer to the block that contains the list of received cells for that message (CLNAP packet).

The block size corresponds to the maximum number of cells that can be received in a packet, which by the CCITT's definition of the CLNAP protocol is 9188 octets or 210 cells ${ }^{|52|}$. Because most files or blocks of data $\mathrm{s}: \mathrm{nt}$ in a broadband environment are likely to be greater than this length, they will need to te segmented into a number of packets. All but the last of these packets will be filled to capacity, meaning that the majority of the message blocks in the header RAM will also be full. Thus setting the block size to this value does not result in any major inefficiencies in RAM utilisation.

Figure 3-9 illustrates the organisation of the message part of the header RAM.

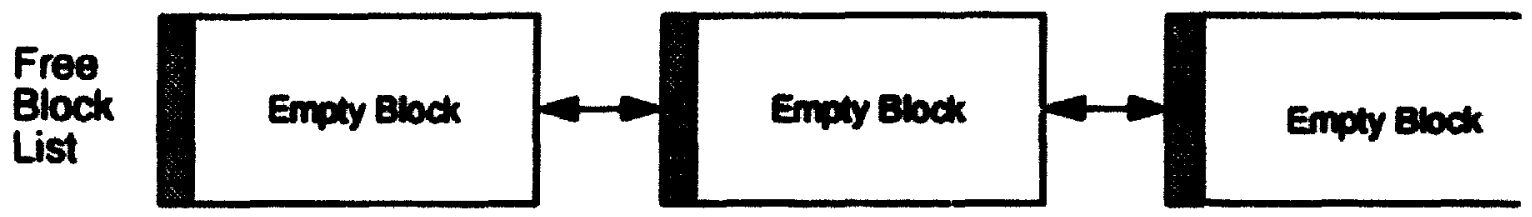

$\cos 1$

Queve

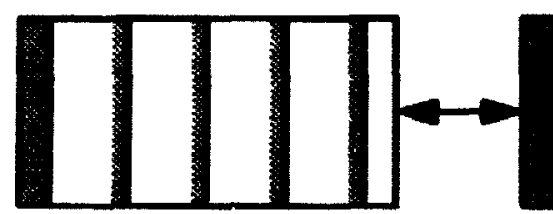

Message Entries
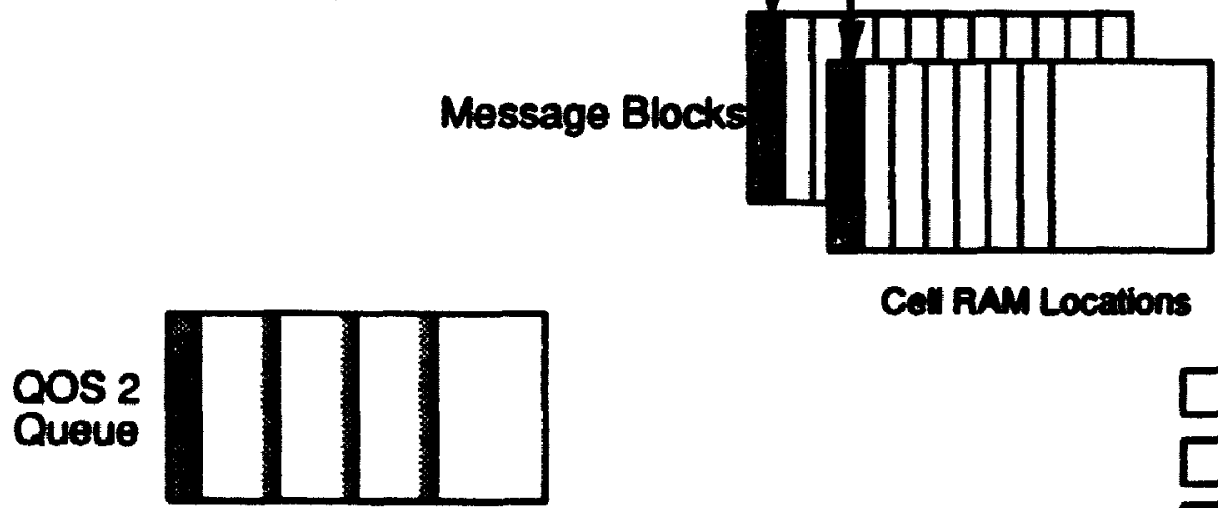

Coll RaM Locations

Pigure 3-9 Organisation of a portion of the message part of the Header RAM.

Entry

Unused

Pointer 


\subsubsection{BOM procedures.}

The first cell of a message to arrive will be a BOM or SSM cell. The MRP first determines which data connection it belongs to by performing a CAM search using the mask in Figure 3-8. This mask searches only the connection identification tields, that is, the VPI. VCI. P and MID fields.

Assuming that the connection has been identified, the MRP then reads the data word for that cell from the CAM memory. It examines the state of the sar_S bit to determine whether it is currently reassembling a message for that process. If the bit value is IDLE. then the MRP proceeds with the new message creation procedures. If the bit value is REASS, meaning that the system is in the middle of receiving another message on that connection, then the MRP aborts the current message using the procedures described in Section 3.6.2.4 and continues with the new message creation.

Before creating a new message, the MRP also tests the state of the CPCS reassembly process for that connection. If there have been no internal errors (e.g. software/hardware faults), then the process should be in the IDLE state. The MRP examines the four octets that make up the CPCS-PDU header, testing the CPI field for validity.

If the CPCS header is valid, then a number of things happen. First, the value of the Btag is stored in the rev_BEtag field of the AC table. Also, the value of the BASize field is incremented by 3 (to allow for the length of any PAD in the trailer) and it is stored in the rev_BASize part of the table. The value of the $\mathrm{LI}$ field is read and stored in the rev_size field.

Next, a free block is found in the header KAM to store the message headers in. The address of the block is written into the AC table, and the cell identifier (its cell RAM location) becomes the first entry in that block. 
Chapter 3: Connectionless Server Architecture.

The CLNAP header fields are then examined. The first parameter checked is the QOS parameter, which is used to determine which message queue the message will be placed in. The CLNAP header is then placed in the appropriate message queue, along with the pointer to the message block, as illustrated in Figure 3-10.

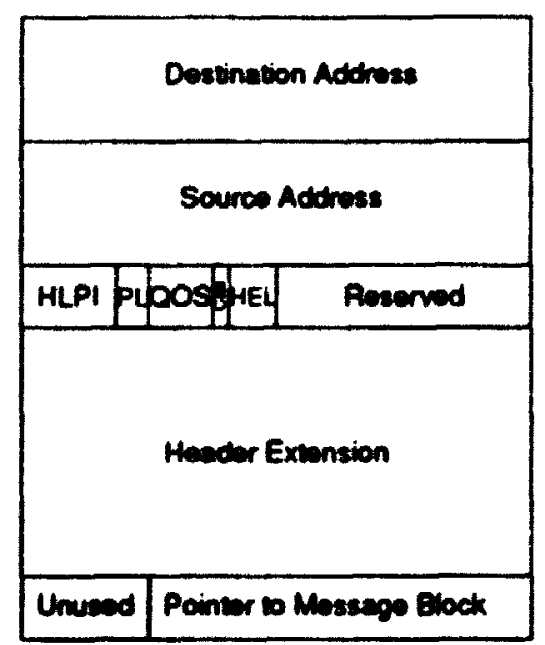

Figure 3-10 Format of QOS queue entry

Once the new message entry has been created, the sequence number (SN) field of the new cell is incremented to equal that of the next expected cell, and is written to the AC table. The SAR and CPCS reassembly processes are both set to the REASS state, and the corresponding sar_S and cpes_S values are written to the AC table. If the cell was a SSM cell, the MRP then proceeds with the EOM procedures described in Section 3.6.2.3. Otherwise, it fetches the next cell header from the header queue and starts again.

\subsubsection{COM procedures.}

The procedures followed upon reception of a COM cell are the most critical to the service rate of the MRP, since the majority of celis received will be COM cells. Thus, it is important that the processing overhead for these types of cells is kept to a minimum.

Assuming a match is found during the CAM search, then the cell is a valid message cell. The first step taken upon reception is to check the state of the SAR and CPCS reas- 
sembly processes for that connection. If all is well. both processes should the in the REASS state.

The amount of data stored in the cell is noted. by adding the cell's I.I tield to the value of the rev_size variable for that connection. The result is compared to the rev_BASize value to ensure that the amount of data received is not greater than that expected.

In the absence of errors, the values of sar_S and cpes_S both remain set to REASS. and are written back to the $\mathrm{AC}$ table along w:th the new value of rev_size. The cell RAM location of this cell is placed in the appropriate message list along with the value of the 1.1 and ST fields, and the header RAM location field in the AC table is updated. If necessiary, a new block is retrieved from the free space list to store the cell location in. The format of the message list block is illustrated in Figure 3-11.

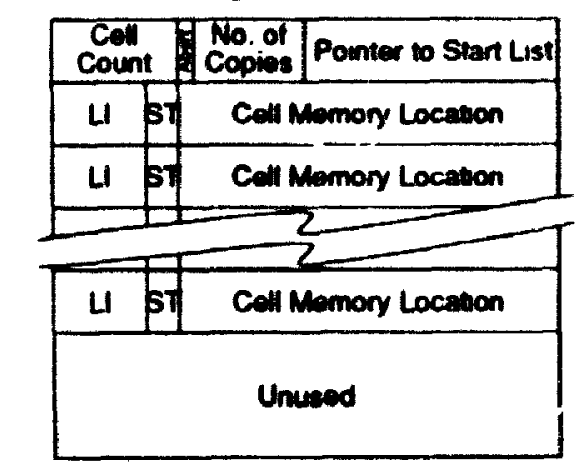

Figure 3-11 Format of message list block.

\subsubsection{EOM procedures.}

The arrival of an EOM cell is treated in a similar fashion to that of a COM cell, except that in addition to checking the rev_size parameter against the rev_BASize value, the $I . I$ field is checked to make sure this is not an ABORT cell.

Next, the CPCS trailer is taken from the header queue and the rev_size value is checked against the Length field. In this way lost and gained groups of cells that aren't noticed by the SAR process are detected. The value of the cell's Elag field is compared to the rev_BEtag field, and the AL field is checked to ensure it contains zeros. 
Whether or not there are errors, the EOM cell will always cause both the SAR and ( PCS processes for that connection to rettirn to the IDLE state ready to receive a new message. The cell RAM location and $\mathbf{L I}$ and ST fields are written to the message list. but only the state variables sar_S and cpes_S need to be updated in the AC table.

\subsubsection{Frror Handling.}

During the processing of the Header queue entries, a number of possible error situations may arise. The follow iing procedures are used to enable the system to react to error situations.

If during the CAM search a match is not found, then the cell belongs to an unknown or unauthorised confiection. The cell is immediately discarded, by removing its entry from the header queue and returning its cell memory address to the Free Space List. The MRP could also notify the System Manager that it received an illegal cell, by placing the cell header in its system queue.

Receipt of an ABORT cell causes the CLSF to discard all untransmitted cells of that message and if necessary issue a new ABORT cell on the appropriate outgoing connection. To enable this to occur, both the SAR and CPCS processes are returned to the IDLE state and the Abort bit of the message block is set. This ensures that both the Routing Processor and Bandwidth Control Processor will realise that the message has been discarded. If the message is already partly transmitted, then this also causes the Bandwidth Control Processor to issue an ABORT cell on the outgoing connection. The MRP then retums all the cell entries in the message block to the Free Space List.

While the SAR process is in the IDLE state, all incoming COM and EOM cells are disarded by the MRP. Thus. placing a connection's SAR process in this state causes all new cells to be dropped until a BOM or SSM cell is received on that connection.

An error at the SAR level. namely a cell sequence error. is detected by an unexpected value in the SN field for COM and EOM cells. An out of sequence cell is discarded and 
the SAR and CPCS reassembly processes for that colinection are both returned to the $I D L E$ state. The location of the message list in the header RAM is found and the Ahort hit of the tirst block in the list is set.

An error at the CPCS level for BOM and COM cells could be either an illegal CPI value or a rev_size value that exceeds the expected rcv_BASize'. Fither of these oxicurrences cause the cell to be discarded and the CPCS process to be put into the $A B O R T$ statte. While in this state, all incoming cells on this connection are discarded until the end of the message is reached, either immediately if this was a SSM cell, or when an EOM cell is received. The current message is also aborted as described above.

For EOM and SSM cells, CPCS level errors also include a non-matching Etag value. an AL field that is not zero, or a rev_size not equal to the Length field. Any of these errors causes the cell to be discarded and the current message to be aborted. For these cells the CPCS and SAR processes always return to the IDLE state.

The MRP also prevents "lost" messages from building up in the system by use at a reassembly timer on each connection. When the SAR process is IDLE, the Time Stamp field in the AC table is set to zero. Every time a cell arrival causes the SAR 10 go to REASS, the Time Stamp field is set to the current value of a counter. Periodically, the MRP steps through all the entries in the table and checks the time stamps. If a non-zern value is found that is too "old", then that implies that a message has not been completed quickly enough and so the message will be discarded.

This function prevents incomplete messages from blocking the transmission of other messages on the outgoing links. This could occur if equipment failure interrupts the message transmission upstream from the CLSF. The time stamp function corresponds to the SAR process reassembly timer defined in Appendix 2 of CCITT Recommendation 1.36.3.

1. This error corresponds to ganed information such as that caused by the accidental cuncatenatiun ef twi, unvergence layer PDUs. resulting from the loss of a multiple of 16 (the $S V$ field maximum value) wells whis h include the $E:(D M$ and BOM cells of two consecutuve messages. 


\subsubsection{Hardware Description.}

The Message Reassembly Processor (MRP) is as shown in Figure 3-12. It consists mainly of dedicated hardware which performs most of the protocol processing, with a RISC processor used for interfacing with the Header RAM and for error handling.

By performing most of the processing in hardware, the MRP can be made to work extremely fast. The hardware devices are capabie of performing all the COM and EOM procedures, as well as the initial BOM and SSM processing. They operate the Active Connection Table, and also carry out the SAR and CPCS reassembly process functions described above.

The processor is called upon whenever a BOM or EOM cell is encountered, or when an error occurs. It is responsible for creating the QOS queue entries, for retrieving blocks from the Free Block List, and for instructing the hardware on where to place incoming cells. When an error occurs, the processor is called upon to tetrieve previously received cells from the Header RAM and return them to the Free Space List, and to adjust the corresponding QOS queue entry.

Refer to Appendix B for the details of how this device is to operate.

\subsection{Routing Processor (RP).}

The Routing Processor is responsible for making routing decisions for each of the received messages. It examines the CLNAP headers stored in the QOS queues in the Header RAM. and performs the CLNAP layer functions, including routing, source address validation, group address (multicasting) support, network charging, and support of multiple levels of Quality of Service. 
Chapter 3: Connectionless Sen er Architecture.

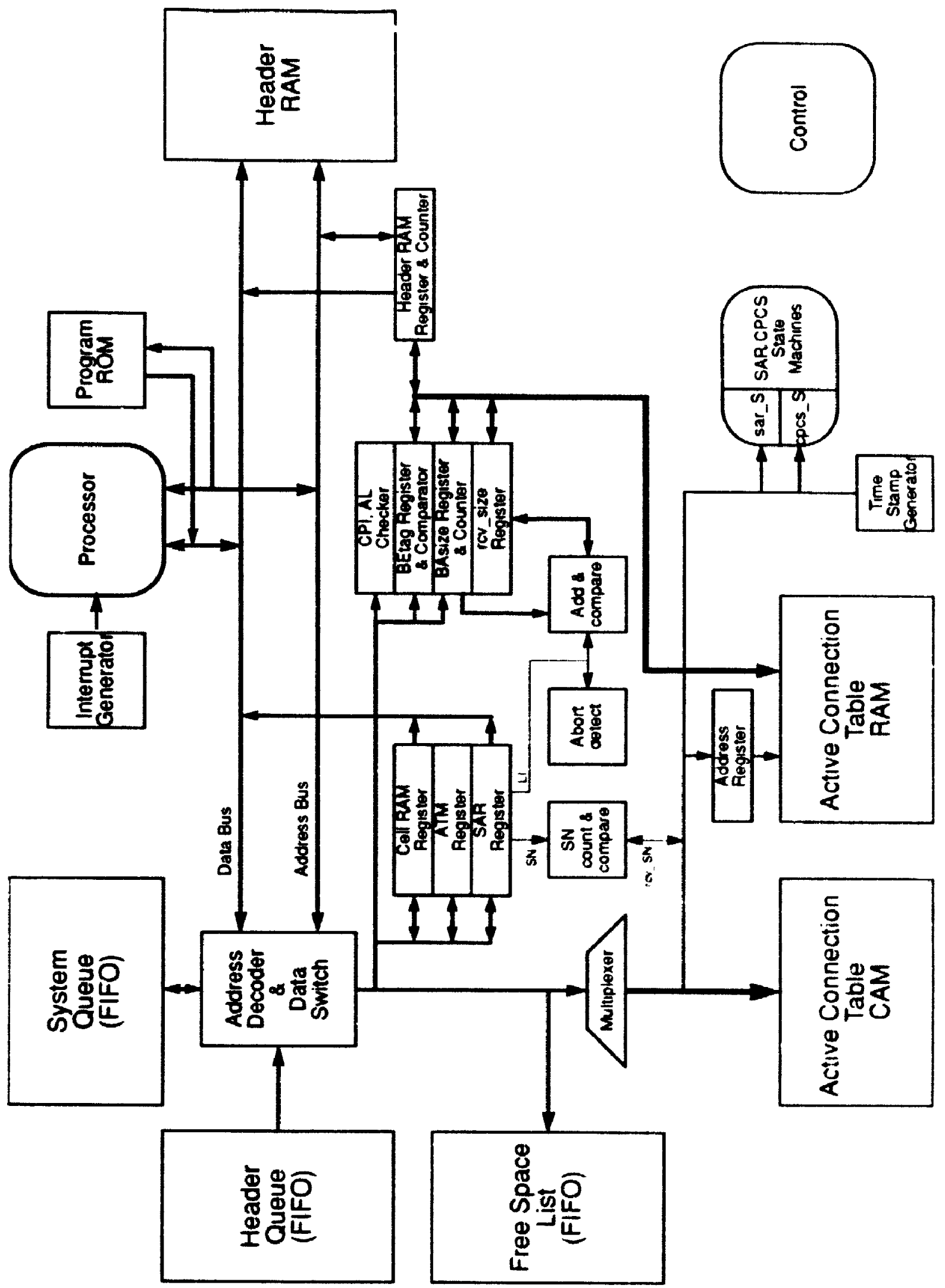

Figure 3-12 Functional Diagram of Message Reassembly Proxcessor. 


\subsubsection{Functions of the RP.}

The most important function of this processor is routing. The RP makes its routing decisions based on the information stored in the Routing Table, which simply maps destination addresses or address ranges to outgoing data connections (i.e. VPI/VCI/MID combinations). The actual traffic routing decisions are made by the System Manager. which monitors network conditions and updates the Routing Table based on its own traffic control algorithms.

\subsubsection{Sequence of operations.}

The RP steps through the various QOS queues and processes message entries. The amount of time spent on each quecie. and the order in which they are selected, is determined by an algorithm chosen so as to give the correct QOS parameters for those messages. That is, the processor removes entries in such a way that the required delay and less (in times of congestion) parameters for the messages are met. The algorithm can be tuned to give the correct values once the overall performance of the system as a whole is known.

When a message entry is read from the queue, the first step taken by the RP is to validate the source address. This will involve some sort of lookup in a validation table, to ensure that cells from this source are allowed. In this case, the validation table used is a 64 bit CAM containing a list of all valid source addresses. The RP simply performs a CAM search on the source address of the message, and checks to see if a match is found. If it is, then the message is accepted.

The processor then conducts a search of the Routing Table for the destination address. Because the E.164 numbering scheme is hierarchical in nature, it is likely that certain ranges of addresses will map to a particular connection, for example all international destinations to one virtual path connection, ail destinations for a particular range of area codes to another. and so on. Also. destination addresses may map to a range of connections, for example an inter-CLSF trunk may occupy an entire virtual path with the virtual 
circuits within being mapped according to availability. As a result, the routing table must be arranged so as to be able to map ranges of addresses to ranges of connections. The routing table operation is described in Section 3.7.1.2.

The eventual result of traversing the routing tree will be a circuit identitier corresponding to the desired destination address. The circuit identitier refers to an entry in a circuit list within the Header RAM, which is then used to tind an outgoing queue to place the message in. Figure 3-13 illustrates the organisation of the outgoing queue part of the header RAM.

Circuit List Connection List
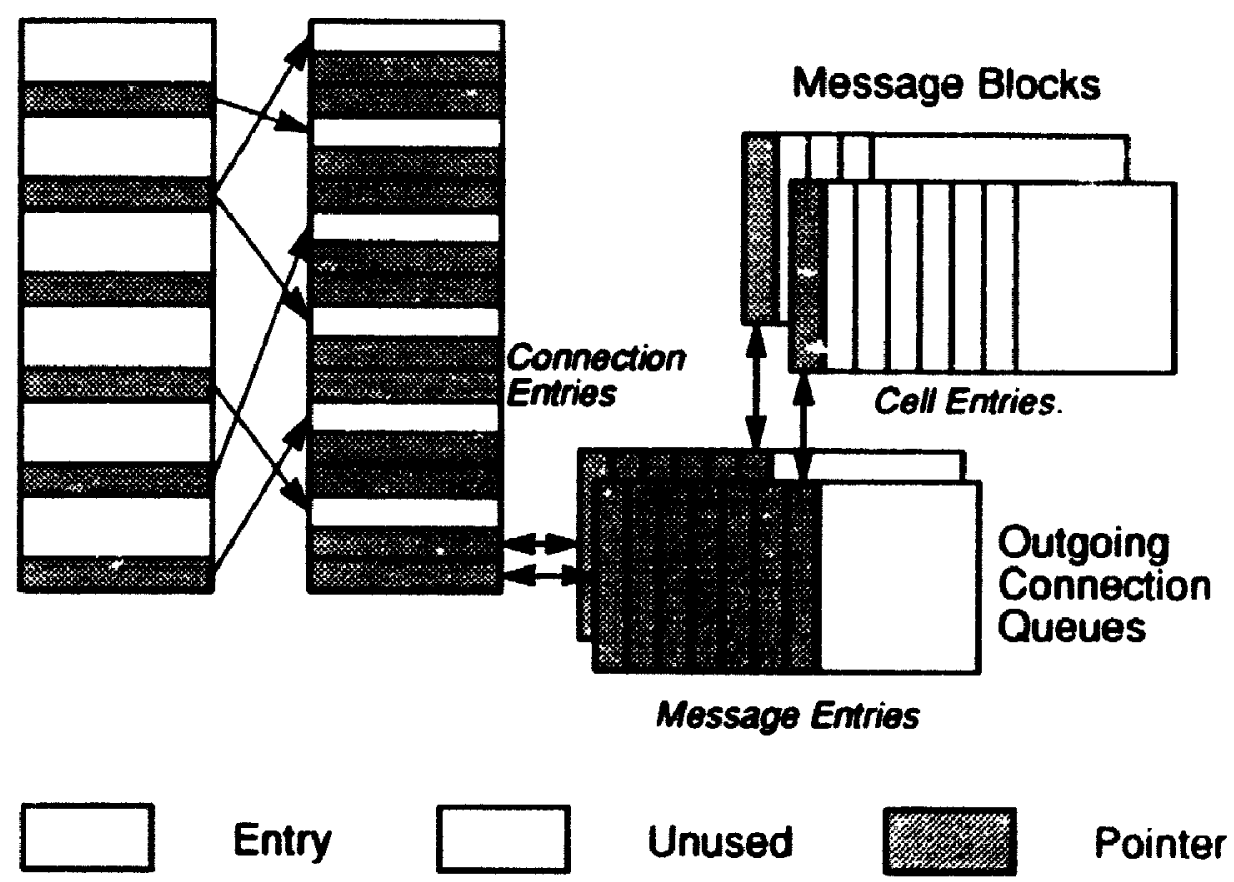

Figure 3-13 Outgoing Quewe portion of the Header RAM

The Circuit List is an area of the Header RAM that contains a list of all known outgoing virtual circuit, virtual path and group connections. The format of each entry in the list depends on what type of connection it refers to. For virtual circuit and virtual path connections, it contains a record of the VPIVCI, the bandwidth allocated to the connection, and a list of pointers to the various data queues associated with the connection. For group addresses, the entry instead points to other circuit list entries that make up the component 
Chapter 3: Connectionless Server Architecture.

connections of the group. The Type field is used to distinguish between the various types of circuit. Possible formats for the circuit list entries are suggested in Figure 3-14.

\begin{tabular}{|c|c|c|}
\hline VPI & $\mathrm{VCl}$ & Type \\
\hline Peak Bandwiduh & \multicolumn{2}{|c|}{ Moen Bandwidith } \\
\hline $\begin{array}{l}\text { Surplus/unused } \\
\text { bandwith count. }\end{array}$ & \multicolumn{2}{|c|}{ Connection ID 1} \\
\hline
\end{tabular}

a) Virtual Circuit connection.

\begin{tabular}{|c|c|c|}
\hline VPI & Unused & Type \\
\hline Peak Bandwoth & Mean Bendwith \\
\hline $\begin{array}{c}\text { Surplug/unused } \\
\text { bandwidth count. }\end{array}$ & Unused \\
\hline \multicolumn{2}{|c|}{ Pointer to list of available VCls } \\
\hline
\end{tabular}

b) Virtual Path connection.

\begin{tabular}{|c|c|c|}
\hline VPI & VCI & Typo \\
\hline Circuit 10 1 & Circuit 10 2 \\
\hline Circuit 10 3 & Circuit 1D 4 \\
\hline Powner to hist of other Crevin 10s \\
\hline
\end{tabular}

c) Group address.

Figure 3-14 Examples of circuit list entries.

The location of the virtual path/circuit entry in the circuit list is that connection's circuit identifier within the system, and is used both by the RP and the Bandwidth Control Processor to refer to the outgoing circuit. SAR layer connections within each circuit are referred to by their connection identifier, which is the location of the entry in the Connection List.

The connection list has an entry for each known outgoing SAR layer data connection. It consists of a list of pointers to the appropriate high and low priority queues (as determined by the P tield) for that connection, and the SAR layer information (SN and MID fields) associated with the queues. If a particular connection has no data to be sent, then the entry for that connection contains a null pointer. The connection list format is illustrated in Figure 3-15.

\begin{tabular}{|c|c|c|}
\hline Sat SN & MO & $\begin{array}{l}\text { No. of quowed } \\
\text { mosisaces }\end{array}$ \\
\hline & \multicolumn{2}{|c|}{ High Priority Cunus Location } \\
\hline $\begin{array}{l}\text { Queve } \\
\text { Start }\end{array}$ & \multicolumn{2}{|c|}{ Low Prionity Queus Location } \\
\hline
\end{tabular}

Figure 3-15 Format of Connection List Entry 
Chapter 3: Connectionless Sul ver Archilciture.

The action that the Routing Processor takes once it has a circuit identitier depends on what type of circuit the identifier refers to. For single outgoing virtual circuits, the processor selects a data connection in the connection list and chooses a queue that corresponds $t)$ the message priority level. The criteria used to select a queue from among the SAR layer connections available will depend on future standardisation artangements. but may involve finding the queue with the least entries, or simply taking a random choice.

If the connection chosen is a virtual path (trunk) connection, the processor may then choose a virtual circuit on that trunk to send the message on. The choice of circuit will depend on the amount of data in the queues, with the message possibly being added to the circuit with the shortest queue. If SAR level multiplexing is allowed on trunk circuits. then another choice must be made as to which connection to use.

For group addresses the processor will have a number of circuits that the message must be transmitted on, corresponding to the various members of the group. A grou! address in this design is simply mapped to a number of ordinary virtual circuit or virtual path connections. These connections are not specifically dedicated to group messages, and so the group message will share an outgoing queue with other non-multicast messages. The processor must visit in turn each of the circuit list entries referred to in the group entry, and place a pointer to the message entry in a connection quete of each one in the same way as described above.

To ensure that the message is not deleted until a copy has been sent on each connection, the number of copies field in the message block is set to the number of circuits in the group. The RP will also create a "start list" area in the header RAM, and put in it a list of all the connection ids that the message has been placed in. The start list field of the message block is set to point to that list. The list is used by the BCP to determine when (o) delete the message from the system (see Section 3.8.1.3 on page 69). 
Once the destination's outgoing connection (or connections) are found for a particular message, the processor creates a new entry in the outgoing queue corresponding to the connection. As the message has by now been completely examined, it remains only to retransmit the message on the outgoing link. Consequently no more of the message's parameters need to be stored, so the outgoing connection queue entry consists only of pointers to the message block. The outgoing connection queve entry is illustrated in Figure 3-19.

\section{\begin{tabular}{|l|l|}
\hline OHser: & Mossage Block Location \\
\hline Firnt Cell
\end{tabular}}

Figure 3-16 Outgoing Connection Queue Entry.

Once the message has been added to the queue, the number of queued messages field in the connection list entry is incremented, and the message entry is removed from the QOS queue.

\subsubsection{Routing Table Organisation.}

There are many ways in which the problem of quickly routing messages could be solved. By way of illustration, one possible method that could be used by this system is dexcribed in this section. It is quite viable to use other routing algorithms, so long as the time taken to find the destination is not too great.

This method is based on the "trie" approach described by T. B. Pei and C. Zukowski ${ }^{|42|}$, with some modifications that make it more suitable to the unevenly hierarchical nature of the E.164 addressing scheme. In this method, the routing table is arranged in the form of a tree. so that the destination address is examined a few bits at a time. Each node of the tree contains a number of pointers, either to other tree nodes or to entries in the circuit list. The tree concept is illustrated in Figure 3-17.

Each node of the tree contains a variable number of pointers, depending on how many bits of the destination address are to be examined at that point. The first word of the node 


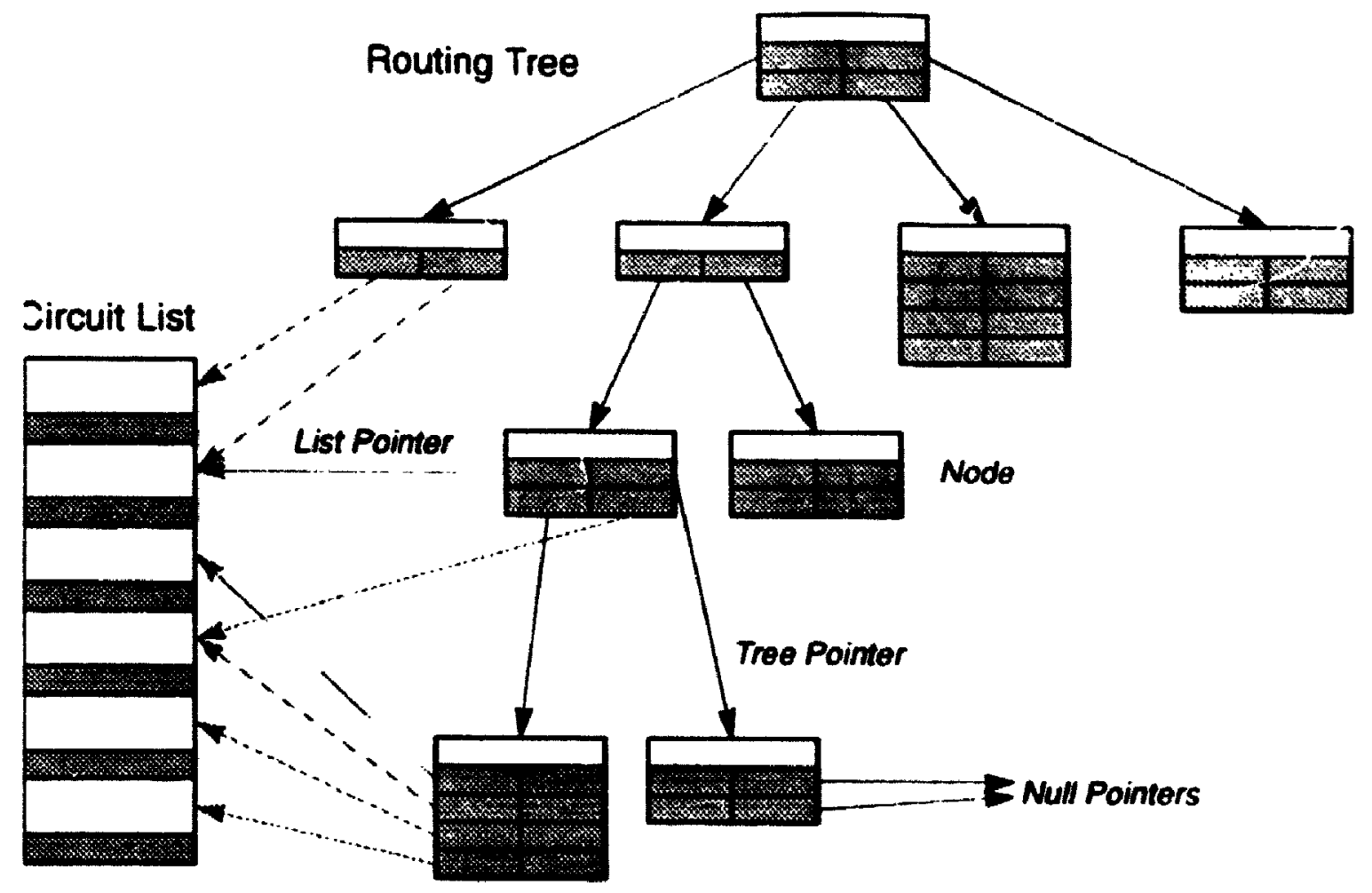

Tigure 3-17 Routing Tree Organisation.

indicates how many pointers are in the node. or how many bits of the destination address are to be examined. Each pointer is a 16 bit address, the most siguiticant bit of which indicates whether the pointer refers to another tree node or to the circuit list (which is lociated in the Header RAM).

To use the Routing Tree, the processor first examines the root node of the tree to determine how many bits to check. It then uses that many bits of the Destination Address 10 select a pointer from the node, and follows the pointer to the next node. The process repeats until a pointer is found which leads to the circuit list.

The speed at which the processor finds the destination depends on the number of bits examined at each node. Unfortunately, examining too many bits at one time requires very large node entries, and results in a significant increase in the overall tree size. When designing a routing tree for a particular local environment, a trade-off must be made between system speed and memory size. The limiting factor is that the mean time taken 
for the RP to complete all its tasks must be less than the mean message transmission time, as will be seen from the simulation results in Chapter 4.

If the processor encounters a null pointer at the end of the tree, then the destination address is a local address which has not been assigned to connectionless data. The processor will abort the message, by sending all the cell RAM locations to the Free Space List. and by notifying the MRP if the received message is incomplete. It then notifies the System Manager, possibly resulting in an "incorrect address" message being sent back to the source.

\subsection{Bandwidth Control Processor.}

The Bandwidth Control Processor is responsible for retrieving the messages from the outgoing queues and providing new headers for the cells so they may be forwarded to the next destination. It does this at a rate corresponding to the allocated bandwidth for that connection. The connection bandwidths are set by the System Manager in response to monitoring information provided by the BCP.

The processor maintains a list of all known outgoing connections and the associated allocated bandwidths for those connections in the Header RAM. At regular intervals it scans through the list, visiting in turn the connection queues for each active connection and transmitting cells from the first message or messages in the queue. The number of cells transmitted from each connection in a given scan depends on the allocated bandwidth parameters for the connection.

As it repeatedly scans the list of connections, the BCP takes note of how often a particular connection has significantly more or less than its allocated traffic capacity. If this occurs on enough consecutive scans, the BCP sends a message to the System Manager asking for the bandwidth reservation for that connection to be changed. The System Manager will issue a request to the ATM control via cells inserted in the outgoing stream. If the 
request is granted. the System Manager will instruct the BCP to alter the allowated bandwidth settings in the Circuit list.

\subsubsection{Procedures Followed by the BCP.}

\subsubsection{Bandwidth Control Procedures.}

The procedures for allocating portions of the outgoing link bandwidth to the outgoing connections are implemented entireiy in software. The processor maintains a record of the allocated bandwidth for each virtual circuit or virtual path connection in the Header RAM. which it uses to determine when and how many cells to transmit on the connection. The exact algorithm that it uses to make the decision is dependent on future standardisation work within the CCITT, as it is yet to be decided exactly what parameters of the traftic will be specified when allocating bandwidth to a connection.

It is possible that the traffic descriptors will specify the mean and peak bandwidths transmitted on the connection. The peak rate on a virtual connection is detined by the CCITT as being the inverse of the time period between the transmission of two consecutive cells on the connection, while the mean bandwidth is the sustained average transmission rate on the connection.

Proposed additions to CCIT: Recommendation $1.371^{\mid 531}$ include examples of a "spacer controller" algorithm for fixing the peak rate on the outgoing connection to the specified value, and a "dual leaky bucket" algorithm for maintaining transmission at the agreed mean and peak rates. It is desirable that the BCP algorithm would incorporate the principles embodied in these two algorithms to adhere to the agreed parameters on the connections.

In order to neet the agreed parameters the BCP must ensure that there is sufficient spacing between consecutive cells to adhere to the peak traftic specification, and that no more than a given number of cells is transmitted in a certain time frame in order $w$ adhere to the mean bandwidth allocation. During that time frame the BCP must process all of the 
currently active ATM layer (virtual circuit) connections and transmit cells on each according to the above restrictions. Because of the processing involved, the outgoing cell stream cannot be created on a cell by cell basis but must instead be assembled in batches in the Header RAM and each batch shifted into the outgoing header queue.

Each batch of output cells corresponds to a complete scan of the outgoing connections by the BCP. The size of the batch will be chosen so that the transmission time of the batch is more than the time required by the BCP to process all the connections.

To assemble the batch, the processor will create a Cell Stream Construction (CSC) area in the Header RAM equal in size to the number of cells transmitted per scan. It then visits each virtual circuit in turn. and using the dual leaky bucket algorithm it determines the maximum number of cells to be transmitted on the circuit during this scan so that the mean bardwidth is not exceeded. A number of cell entries up to the maximum is copied from the messag e blocks in the circuit's connection queues to the CSC area.

The amount of data transmitted on each SAR layer connection within the virtual circuit will depend on how the circuit's bandwidth is to be split up among those connections. The division of bandwidth between the connections, including between the high and low priority connections, is in the words of the CCITT "for further study" [52].

The placing of the cells in the CSC area uses the spacer controller algorithm to determine the interval between cells. Basically, the BCP starts from the beginning of the area and searches for an unused slot, into which it places a cell. It then jumps ahead a number of slots corresponding to the peak bandwidth, and starts searching again. The result is that the connection bandwidth is not greater than the peak, although it may be less.

Once all the connection queues have been processed in this way, the CSC area will contain the outgoing header queue entries of all the cells to be transmitted during the scan. in chronological order. Unused locations in the area will contain idle cell entries. The BCP processor now signals its DMA hardware to begin shifting the entire CSC area to the out- 
put queue (see Section 3.8.2). While it is doing this the BCP may create another CSC area and begin processing for the next scan, once the current scan time interval is up.

If during the processing of a circuit the end of the CSC area is reached thefore all the cells have been placed. then this means that the circuit's mean bandwidth cannot ike upheld in this scan without violating the peak parameter. This will only oxicur in times of high traffic load, when cells from other circuits have already taken up most of the available slots. The unused portion is recorded and added to the circuit's allocation in the next scan, so that the overall mean level can be maintained

The unused portion is also added if the circuit's queues empty before the current allocation is used up (i.e the circuit has less cells in this scan than required to meet the mean). If the allocation was discarded then the actual mean outgoing bandwidth on that circuit would be less than the agreed setting, possibly resulting in congestion on the circuit when a later burst of traffic arrives.

The result is that in a given scan some circuits may have quite large allocations left over from previous scans. If these circuits are processed first, they may use up a considerable portion of the scan's slots and cause other circuits to have their allociations held over. To prevent this mechanism from causing an uneven quality of service among the circuits, the scan order must be changed from scan to scan. A possibility is to make the starting point of the scan rotate between the circuits.

As the BCP repeatedly scans the active connections, it makes a note of the number of times a circuit does not have enough cells to meet its allocation, or has a large number of packets in the queue. If a circuit falls outside these limits more than a predetermined number of successive cycles, then the BCP sends a message to the System Manager asking it to lower or raise the agreed mean bandwidth for that circuit.

Once a circuit has had no data to send for a sufficiently long period of time, its mean bandwidth allowance is reduced almost to zero. Some residual mean allowance is main- 
tained to ensure that the data does not build up excessively when a new burst of messages arrives.

\subsubsection{Transmission Procedure.}

In order to transmit a cell from the header RAM. the BCP must produce appropriate SAR layer header information and place an entry for that cell in the outgoing Header Queue. To aid in doing this the processor effectively maintains a SAR ser-er process (as detined in Recommendation 1.363 ) for each active outgoing SAR layer connection. No CPCS process is needed. as the CPCS layer protocol information is stored in the cell RAM and is retransmitted unchanged from the way it went in.

When the BCP decides to take a number of cells from a particular connection, it first reads the connection's entry in the connection list to determine the connection's identifier and the location of the message queues. The first message entry in the queue is then used to find the appropriate message list and the location of the first untransmitted cell in the block. The high priority queue is visited first. followed by the low priority queue.

The BCP proceeds to take a number of cells from the list and generate outgoing header queue entries for them. To do this, it needs to generate a correct sequence number (SN)

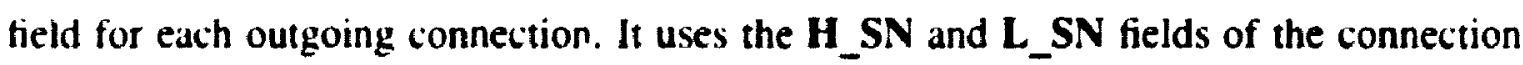
list entries to maintain a record of the current sequence number on the high and low priority queues of each connection. After each cell has been placed in the CSC area, the SN tield for that queue is incremented by one modulo 16 .

The formats of the outgoing header queue entries are illustrated in Figure 3-18. Inilially. the BCP writes the cell RAM location to the CSC area. The Discard field is used to instruct the Outgoing Cell Linit whether or not to discard the cell entry from the cell RAM - necessary when multicasting of messages is supported. The VPI and VCI fields are written to the queue. and an appropriate Payload Type (PT) and Cell Loss Priority (CLP) fiekls are added. These $u$ ill most likely be some preset value for connectionless data. It 
Chapter 3: Connectienless Servier Architecture.

then takes the $\mathbf{L I}$ and $\mathbf{S T}$ fields from the message list. combines them w ith the appropriate SN and MID fields from the connection list and the P field according to what queut it is working on. and writes them to the CSC area.

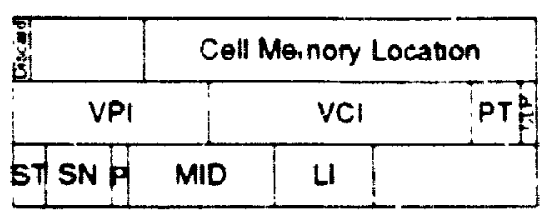

a) Data cell entries

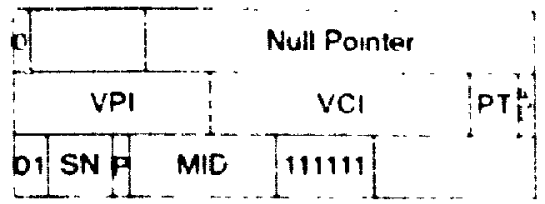

b) Abort cell entry

Figure 3-18 Outgoing Heider Qucuc Entnis

After the cell entries have been placed in the CSC area. the Queue Start ticeld of the message's outgoing queue entry is updated to point to the new first cell in the hlock. If the block is empty, it is returned to the free block list.

\subsubsection{Multicasting procedure.}

When the BCP processes a multicast message, it must ensure that each cell is nut removed from the system until a copy has been sent on every connection in the group. The BCP must therefore keep track of how many cells of the message have been sent on each sub-connection. This is done through use of the number of copies and start list fields in the message list block (see Figure 3-11 on page 53).

If the number of copies field has a value greater than one, then the message is multicast. The BCP uses the start list field to find a the location in the RAM of a list of all the connections in that group (the list is created by the RP when it processes the message). The start list contains a list of all the connections in the group, and the number of cells transmitted so far on that connection.

The BCP scans the iist until it tinds the entry corresponding to the connection it is working on at that time. It then compares this value with all the others in the group. If the current connection has transmitted the least number of cells, then the difference between this and the next least connection is the number of cells that are being transmitted for the 
last time. The BCP will set the Discard bit of this number of cells when it places them in the CSC area. Otherwise, the Discard bit is set to zero.

When the EOM cell of a message is transmitted. the number of copies field is decremented. Once the value of this field reaches one, the start list is removed from the header RAM and the message becomes identical to a normal message (i.e. all the remaining cells are discarded after they are uransmitted. and the message block is removed after all cells have been sent).

\subsubsection{Abort cell procedures.}

An aborted message will have the Abort bit set ir. the message block. This bit was set by the MRP when an error occurred. When the BCP discovers an aborted message, it places an ABORT enu y in the CSC area (see Figure 3-18 on page 69). The ABORT cell is treated just like an EOM cell, causing the message block to be removed if the message has one destination, or causing the number of copies field to be decremented for a multicast message.

\subsubsection{Setup and Maintenance Procedures.}

In order to be able to transmit cells on an nutgoing connection, the BCP first needs to know the allocated bandwidth for that connection. This implies that the connection must actually have an allocated bandwidth. At system setup time, the System Manager must request from the ATM control a bandwidth allowance for each outgoing connection that is established.

As each new allocation is made, the system informs the BCP by leaving a message in the System Queue. The message consists of four data fields - the connection identification number. a control field. the new mean bandwidth and the new peak bandwidth. The connection ID number refers to the connection's location in the connection list (in the Header RAM). The control tield is used to indicate what type of message this is (in this case a 
Chapter 3: Connectionless Server Architecture.

bandwidth specification). The format of the system queue entry is as shown in Figure 3. 19.

\begin{tabular}{|c|c|}
\hline Controf & Connection 10 \\
\hline Peak BW & Mean 8W \\
\hline
\end{tabular}

Figure 3-19 System Quede Bandwidh Speciticadion Entry.

\subsubsection{Hardware Description.}

The functional diagram of the BCP is given in Figure 3-20. It consists simply of a pro cessor with associated memory, and a DMA unit for moving cell information from the Header RAM to the header queue.

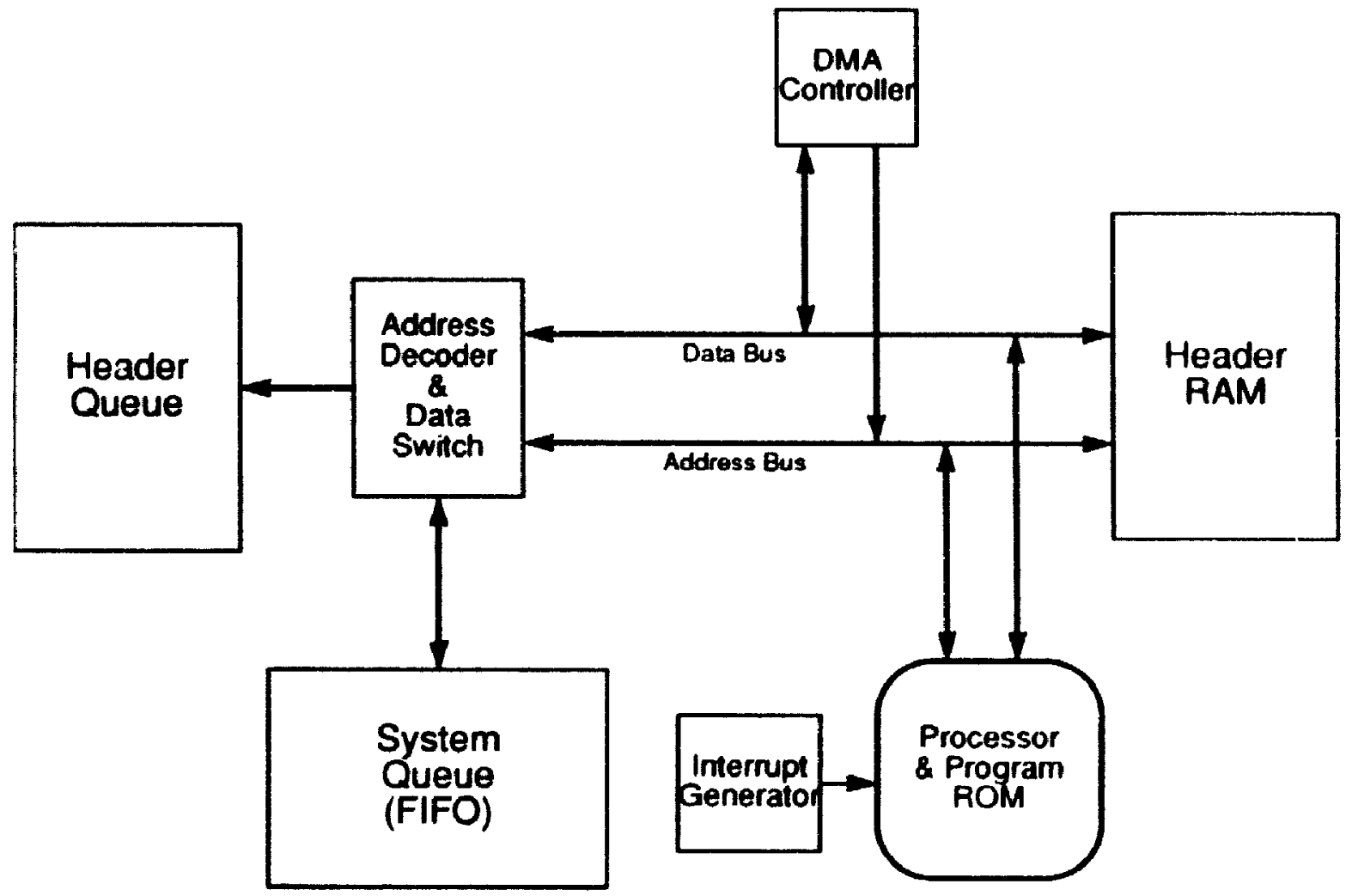

Figure 3-20 Functional Dagram of the Bandwidth Control Processor.

The processor's data and address buses are connected directly to the Header RAM, in the same way as for the Message Reassembly Processor. Also connected to the buses is the address decoder/data switch unit. This device interfaces the header and system queues 
to the processor. The processor sees the system queue and the header queue as memory addresses, because the address decoder recognises the addresses as the processor accesses them and activates the appropriate device.

When the BCP decides to transmit a batch of cells, it loads the DMA controller with the address and size of the CSC area and initiates the transfer of the block. The controller works in cycle stealing mode, where the transfer of a data word occurs while the processor is executing an instruction that doesn't involve memory access. When the processor finishes its scan and is idle, it should go into a loop that ensures the DMA is granted complete access to the bus, so as to ensure that all the cell entries are transferred befcre the scan time is up.

The act of placing an entry in the system queue generates a processor interrupt, causing the processor to read the two data words from the System Queue via the data switch, and use them to update the connection's entry in the header RAM.

\subsection{Outgoing Cell Unit.}

The outgoing cell unit is responsible for removing entries from the Outgoing Header Queue, retrieving the cell bodies from the header RAM, and reassembling the cells for transmission on the outgoing link. It operates in synchronisation with the outgoing cell stream.

\subsubsection{Procedures followed by the OCU.}

At the start of each cycle, the OCU checks its system queue to see if there are any system cells waiting to be transmitted. If there is, it will simply take each word in turn and feed it straight to the outgoing framer circuit. It is up to the System Manager to ensure that the cells it places on the outgoing links are valid. 
If there are no system cells to transmit, the OCU then checks the outgoing header queue for entries. If the queue is empty, the OCU inserts an idle cell on the outgoing link (identified by a standard VCI/VPI pattern of all zeros).

When there are cells to be transmitted, the header queue contains entries in the format illustrated in Figure 3-18 on page 69. The OCU first fetches the cell memory lixution from the header queue and places it on the cell RAM address bus to retrieve the first word of the cell payload. It then fetches the second header queue word which contains the ATM header, and passes it through a CRC generating circuit which generates the header HF:C field. The header is passed octet by octet to the framing circuit and on to the outgoing physical link.

The last header queue word is fetched and the first two octets (the SAR layer header) are passed through a SAR layer CRC generating circuit, then on to the framer. The SAR layer trailer (LI field) is buffered for later transmission. The first word of the cell body is fetched from the cell RAM, and it too passes through the CRC generator and on to the framer. The R AM address is incremented step by step, until all 11 cell words have been read and transmitted. The $\mathbf{L I}$ field then passes into the CRC generator, and the value of the CRC field is read from it and transmitted. Finally, the original cell RAM address is placed on the Free Space List if the Discard bit is set.

If the cell was an ABORT cell, then the cell memory location is invalid and $s$ the OCU must supply the cell payload, which for an ABORT cell is simply a string of zeros. All the othe: procedures are the same as for data cells. An option would be not to have an invalid pointer, but rather a pointer to a special cell RAM location that is known to crntain only zeros. In that case the ABORT cell would be treated exactly the same as the other cells.

Idle cell entries in the header queue are recognised by their VCI/VPI pattern, and cause the OCU to generate an idle cell on the outgoing link. 


\subsubsection{Hardware Description.}

The functional layout of the outgoing cell unit is illustrated in Figure 3-21. This unit is intended to follow the procedures described in the previous section.

The OCU operates on a cyclic basis, in synchronisation with the outgoing cell stream. Header and data words are taken one at a time, converted to eight bit words and fed into the framer circuit. A multiplexer and latches are used to reconstruct the cell from the header entries and the data stored in the cell RAM. Cyclic redundancy check fields are generated from the cell information and added into the appropriate parts of the cell as it is transmitted.

The unit is a hardware device, controlled by a tinite state machine or other such hardware control mechanism. It contains a couple of counters, some buffers, a comparator, a multiplexer and some CRC generators. It is well suited for custom or semi-custom VLSI implementation.

Refer to Appendix $\mathrm{C}$ for the details of the operation of this unit. 
Chapter 3: Connectionless Server Arthilecture.

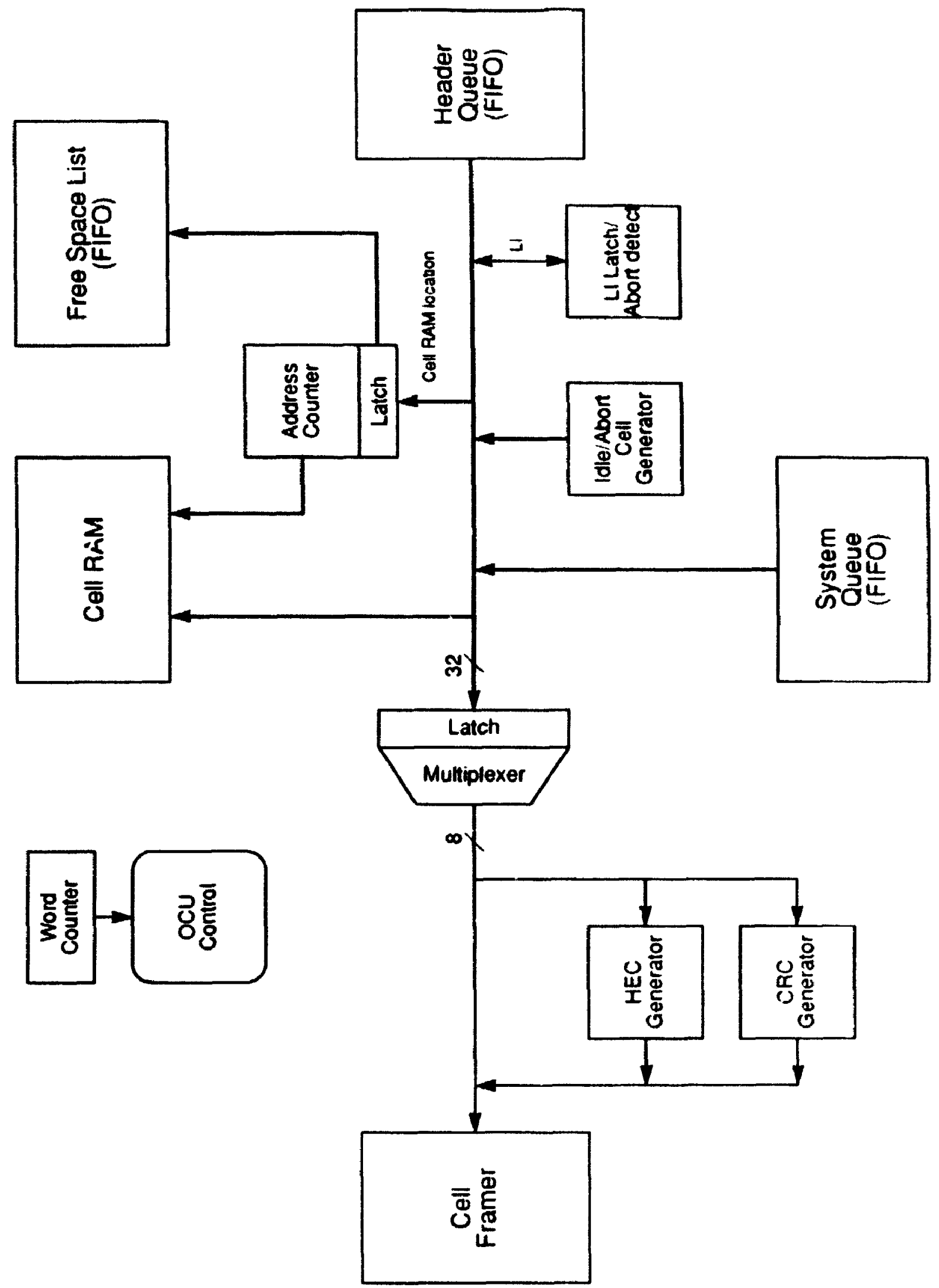

Figure 3-21 Functional architecture of the Outgonng Cell Umt. 


\section{Chapter 4: Experimental Results.}

\subsection{Approach Taken.}

In order to judge the performance of the CLSF design, a simulation program was written in the $C$ language to model the operation of the system.

To set up the program, a number of assumptions needed to be made about the various operating parameters of the system, such as the execution times for the processor algorithms. In order to evaluate the sensitivity of the results to these assumptions, a number of simulation runs were made to determine the effects of changes in the parameters.

Default values of all the parameters were selected, then a particular parameter was chosen and allowed to vary over a range of values while the others remained co.stant. The resulting changes in the system were plotted against the parameter of interest. This was done for each parameter that was either significant to the system or which had a default value that was arbitrarily chosen.

By varying the parameter values in this way it was possible to judge both the significance of the choice of default, and the range of values within which the parameter must lie in order to ensure reliable system operation. This is particularly useful when it comes to parameters such as software execution times, as it provides a working range within which any potential algorithm must be capable of operating.

The end result of this process is an indication of whether or not the design as it stands is capable of performing the required functions of a CLSF at the two standard link rates of $155 \mathrm{Mbps}$ and $622 \mathrm{Mbps}$, and what limits must be observed by the software algorithms. It also gives an idea as to what kinds of packet delays can be expected to be seen by data travelling through the system. and how much data can be expected to build up in the system under normal conditions. 
The following sections describe the default settings used in the molel. and the results obtained from the simulation runs. The way in which the simulation program actually models the design is detailed in Appendix D.

\subsection{Default Parameter Values used.}

\subsubsection{Input Traffic Parameters.}

The input traftic to the system takes the form of a number of sources sharing a common input link using ATM multiplexing. The link is capable of running at either $1.55 \mathrm{Mhps}$ or $622 \mathrm{Mbps}$.

The bandwidth of the link is divided in such a way that all of the sources have the same mean bandwidth allocation. $b$. When expressed as a proportion of the link capacity. the sum of the mean bandwidths of all the sources equals the desired load on the system p. such that the value of $\rho$ is between zero and one ${ }^{\prime}$. In order to evaluate the system under maximum load, the default value of $p$ is 0.999 .

Each source transmits data in bursts, followed by an idle period. The bursts correspond to the transmission of files or blocks of data, and their lengths are exponentially distributed and rounded up to the nearest cell. The idle period between bursts is such that the allocated mean bandwidth of the source is maintained. Some of the bursts are multicast to a number of destinations.

The bursts are divided up into CLNAP layer packets less than or equal to the maximum size, and the packets sent as messages through the CLSF. It is assumed that the AAI, layer is operating in Message mode (see Section 2.3.1.2 on page 23). Packets are split into

1 Settng the value of $r$ " be exactly equal to enther () or 1 causes the simulatun program in give inacurale results dise to too much or too liftle traffic on the input. so a value between these twr) limits is used 
Chapter 4: Experimental Results.

cells, and the cells multiplexed on the input link. There exists a certain probability that a given cell will have bit errors or be dropped or misinserted into the cell stream.

Table 4-I lists the default values of the input traffic parameters used during the simulation runs.

\begin{tabular}{|l|l|}
\hline \multicolumn{1}{|c|}{ Parameter } & \multicolumn{1}{c|}{ Value } \\
\hline Cell transmission time $T_{c}$ & $\begin{array}{l}2.74 \mu \text { (at } 155 \mathrm{Mbps} \text { ) } \\
682 \text { as (at } 622 \mathrm{Mbps} \text { ) }\end{array}$ \\
\hline No of sounces $N$ & 20 \\
\hline Input Traffic Load $\rho$ & 0.999 \\
\hline $\begin{array}{l}\text { Connection mean hand- } \\
\text { width } b\end{array}$ & $\frac{\rho}{N}=0.04995$ of capacity \\
\hline Mean burst length I & 600 cells \\
\hline Maximun packet length & 210 cells \\
\hline Multicast probability & 0.0001 \\
\hline Maximum multicast size & 4 destinations \\
\hline Cell Error Prohability & 0.00001 \\
\hline
\end{tabular}

Table 4-I Input traffic parameter values

\subsubsection{Input/Output Parameters.}

The only parameters of the input and output systems that are not explicitly specified by the design are the maximum permitted input and output header queue lengths. The default values of these parameters used in this experiment are listed in Table 4-2. The Cell RAM size is considered to be unlimited for the purpose of this exercise.

\begin{tabular}{|l|l|}
\hline \multicolumn{1}{|c|}{ Parameter } & \multicolumn{1}{|c|}{ Value } \\
\hline \hline Maximum input queue size & 10000 cells \\
\hline Maximum output queue size & 10000 cells \\
\hline
\end{tabular}

Table 4-2 Input and output paraneters. 


\subsubsection{MRP Parameters.}

Table 4-3 lists the default values of the parameters related to the Message Reasisembly Processor. They all refer to the times taken for the MRP to perform the tasks listed in Section 3.6 .2 on page 49 .

\begin{tabular}{|l|l|}
\hline \multicolumn{1}{|c|}{ Operation } & \multicolumn{1}{|c|}{ MRP Processing Time } \\
\hline \hline Process BOM cell & 33 MO ns (software) \\
\hline Process COM cell & $330 \mathrm{~ns}$ \\
\hline Process EOM cell & $363 \mathrm{~ns}$ \\
\hline Process SSM cell & BOM + 132 ns \\
\hline Discard Incoming Cell & $264 \mathrm{~ns}$ \\
\hline Remove QOS Entry & $1650 \mathrm{~ns}$ (software) \\
\hline Remove Cell from RAM & $264 \mathrm{~ns}$ (soffware) \\
\hline
\end{tabular}

Table 4-3 MRP Cell Processing Times

The information in Table 4-3 was obtained from the hardware timings given in Appen-

dix B, based on a clock period of $33 \mathrm{~ns}$. This period was chosen as theing representative of current technology, as there are many processors available today that are capable of running at $30 \mathrm{Mhz}$ or faster (an example is the SPARC series of RISC processors). In all probability the true times would be slightly lower, as future advances in technology will bring about faster hardware and faster process. .s, but by erring on the side of caution it is ensured that conservative results are obtained.

The software times were based on an estimate of the number of machine instructions needed for the processor to perform those tasks. It was assumed that on average the processor requires two cycles per instruction, again erring on the side of caution as future RISC processors using instruction caches will be capable of better than that.

Naturally the true software execution times will vary from these defaults, as they depend both on the processor being used and the exact algorithm chosen. Part of the purpose of the simulation runs will be to determine the effect these times have on the system. 
and to determine the limits within which the algorithms must operate in order to meet sys. tem requirements.

\subsubsection{RP Parameters.}

Table 4-4 lists the default processing times taken by the Routing Processor to perform its main tasks. The queue scan parameter is the time taken by the RP to scan through the eight OOS queues when all of them are empty. so that this is the time taken for it to notice when a new packet is placed in the queues. The process packet time is the time taken by the RP to make all the required address validation and routing decisions required of a single packet, and to transfer the packet entry from the QOS to the outgoing connection queues.

\begin{tabular}{|c|c|}
\hline Operntion & RP Processing Tibe \\
\hline Queve Scan & $1.32 \mu \mathrm{s}$ (software) \\
\hline Process Packet & $66 \mu \mathrm{s}$ (sofiware) \\
\hline
\end{tabular}

Table 4-4 Routung Processor execution times.

The same assumptions made in the case of the MRP also apply to these values. Again. the simulation runs will determine the sensitivity of the system to these parameters, and will also determine the limits within which the real system must operate.

\subsubsection{BCP Parameters.}

The default parameters relating to the Bandwidth Control Processor are as listed in Table 4-5. The scian rate and peak bandwidth parameters refer to the bandwidth control algorithm detailed in Section 3.8.1.1 on page 65. The connection processing time refers to how long the BCP takes to process a single connection queue, while the copy time is how long the processor requires to copy a single cell entry from the message block to the cell stream construction area. The hardware shift time is the time taken by the BCP DMA hardware to uansfer a cell from the header RAM to the outgoing header queue. 


\begin{tabular}{|c|c|}
\hline Parameter & Value \\
\hline BCP scan size & Ifx(n) cells per kidu \\
\hline $\begin{array}{l}\text { Peak Connection Band- } \\
\text { width }\end{array}$ & $4 \times$ Meat Bindwith \\
\hline $\begin{array}{l}\text { Connection Processing } \\
\text { lime }\end{array}$ & $33 \mu s($ witlwate) \\
\hline Cell copy time & $330 \mathrm{~ns}$ (software) \\
\hline Hardware shift time & $247 \mathrm{~ns}$ \\
\hline
\end{tabular}

Table \&5 Parameters used in BCP procesurer inulel.

The same assumptions made for the RP and MRP also apply to the tinkes livfed in this table.

\subsection{Accuracy of the results.}

The purpose of performing the simulations was to evaluate the perfirmance of the system model under various input traftic conditions and system model paranketers. In order for the results to be useful, however, it was necessary to get some idea of the aciuracy of the results obtained.

To obtain a quantitative idea of the aceuracy of the simulation results, it was mecessary to repeat each simulation run a number of times. From the spread of the results obtained, it is possible to build up a $95 \%$ contidence interval for each data point.

The $95 \%$ confidence interval is calculated as follows:

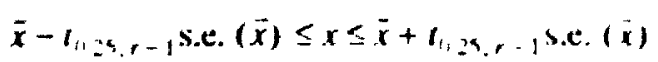

where $x$ is the true value of the result, $\bar{x}$ is the mean of the observed results, $r$ is the numler of runs, $t_{025, r-1}$ is the 95th percentage point of a student's $t$ distribution with $(r / 1$ degrees of freedom, and s.e. $(\bar{x})$ is the standard error of $\bar{x}$, calculated as follows:

$$
\text { s.e. }(\bar{x})=\sqrt{\frac{1}{r(r-1)} \sum_{i=1}^{\prime}\left(x_{1}-x\right)^{2}}
$$



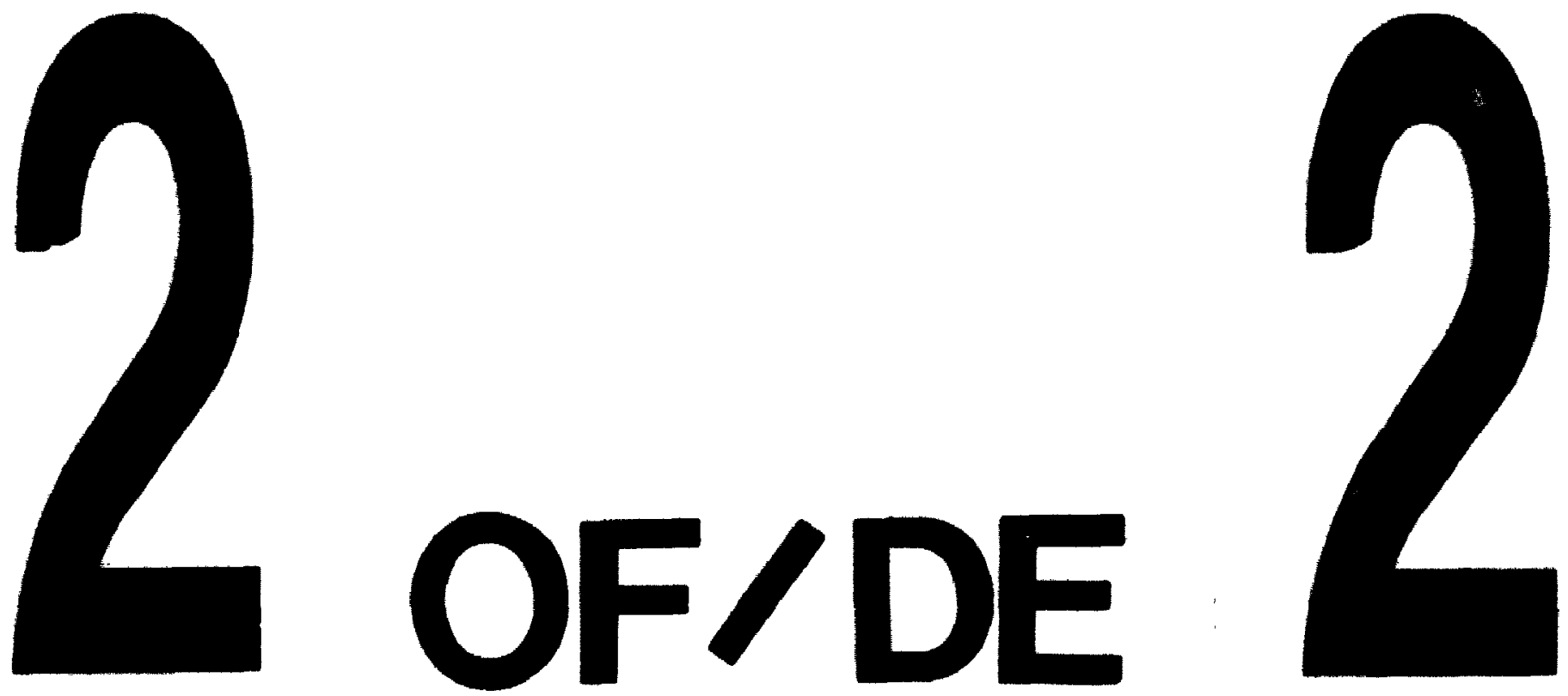

PM-1 31\%" "X4" PHOTOGRAPHC MICROCOPY TARGET Nas rotoa AMSI/RO *2 EOUVALENT

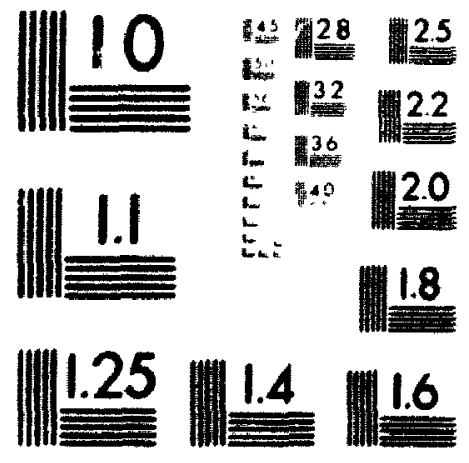


where the $x_{i}$ 's are the individual observations.

Once the confidence interval has been established, the next step is to minimise it so as to obtain accurate results. The interval can be reduced most effectively by reducing the standard error of $\bar{x}$, either by increasing the number of runs $r$, or by increasing the simulation length so as to reduce the spread in the results.

In order to reduce the confidence interval as much as possible, the number of runs per simulation was set at 8 . This reduces the standard error without causing the simulation execution time to be too great. Each set of results took about 30 minutes to produce, so that a 20 point data plot could be obtained in about 10 hours.

Since under some parameter values data may build up in the system, resulting in a buildup of packets in the simulation model, it was desirable to keep the simulation length as low as possible to avoid high memory usage on the workstations. A number of trial runs were made using the default parameter values, and it was discovered that a simulation length of 5 secionds at $155 \mathrm{Mbps}$ or 1.5 seconds at $622 \mathrm{Mbps}$ produced a $95 \%$ confidence interval in the processor usage and queue length results of less than $\pm(1) .36 \%$ of the mean. while the delay and RAM contents had a confidence interval less than $\pm 4.2 \%$. These variations were deemed sufficiently low, and so the above simulation lengths were used in the simulation runs. The lengths correspond to the passage of about 2 million cells through the system.

It was also thought that initialisatior. conditions might introduce some bias error into the results, so a test run was made wher: the simulation period was broken down into a series of short batches. The system statistics were collected over each batch period, using batch lengths of 0.5 seconds at $155 \mathrm{Mbps}$ and 0.1 seconds at $622 \mathrm{Mbps}$. It was observed that at both link rates the system results took three batch periods to stabilise at their final values. This bias error introduced by the initial conditions was removed by collecting sys- 
tem statistics only after 1.5 seconds had passed at $155 \mathrm{Mbps}$, and after 0.3 seconds at 622 Mbps.

These procedures have ensured that all random errors have been reduced and/or quantified in all the simulation results. All results obtained should therefore accurately reflect the conditions of the simulation model within the confines of the $95 \%$ confidence intervals, so that any errors present are due only to inaccuracies in the model itself.

\subsection{Results.}

Each simulation was intended to illustrate the effect on the system of a variation in one of the above mentioned default parameters. The following subsections detail the results of interest from some of the simulations, and explain what inferences can be made about the system from those results.

For each simulation a suitable range of values for the parameter was chosen, and the simulation set to produce a set of results for 20 values of the parameter within the range. The software package Matlab was used to produce plots of the resulting curves.

Table 4-6 lists the statistics output by the simulation program. Entries marked by an asterisk $(*)$ include a $95 \%$ confidence interval.

\begin{tabular}{|l|l|}
\hline \multicolumn{1}{|c|}{ Packe/Cell Statibtics } & \multicolumn{1}{|c|}{ Trafic Statistics } \\
\hline $\begin{array}{l}\text { Average Packet Delay* } \\
\text { Average Cell Delay* } \\
\text { Average Packet Delay by QOS }\end{array}$ & Measured Input Load \\
\hline
\end{tabular}

Table 46 Results output by the simulation program. 
Chapter 4: Experimental Results.

\begin{tabular}{|l|l|}
\hline \multicolumn{1}{|c|}{ Packet/Cell Statistics } & \multicolumn{1}{c|}{ Traffic Statistics } \\
\hline \multicolumn{1}{|c|}{ Queue Statistics } & \multicolumn{1}{|c|}{ Processor Use Statistics } \\
\hline \hline Average Input Queue Length* & Average MRP processor usige* \\
Maximum Input Queue Length & Average RP processor usage* \\
Average Cell RAM contents* & Average BCP processor usage* \\
Maximum Cell RAM contents & \\
Average Header RAM contents* & \\
Maximum Header RAM contents & \\
Average Output Queue Length* & \\
Maximum Output Queve Length & \\
Average QOS Queue Lengths & \\
Average Connection Queue Lengths & \\
\hline
\end{tabular}

Table 4-6 Results output by the simulation program.

Due to the nature of this broadband system, the packet delays mentioned here are not defined as in the conventional sense of the term. Because the packet can begin retransmission before it has been completely received, there is no specific time at which it can be said that "the packet has arrived in the system" and "the packet has departed the system." Instead, the packet delays presented here are defined as the time interval between when the first cell of the packet enters the system, and the last cell of the packet leaves. In this way both the delays due to the routing overhead and the delays due to cell processing can be included in the measurements.

Note that this implies that the packet delay is dependent on the packet length. As mentioned in Section 3.6 .2 on page 49, however, the burst length is normally such that most packets are at their maximum length, and so except where the burst length is varied this dependence on packet length does not affect the packet delay result.

In each the following sections only a subset of the results is presented, those that are actually affected by the parameter of interest. 


\subsubsection{Effect of the input traffic parameters.}

The intent of these simulation runs was to highlight the effect on the model of the various parameters of the input traffic.

\subsubsection{Traffic Luad.}

This simulation showed how the system coped with various levels of traffic on the input link, by varying the traffic load across its range.

The steadily increasing traffic load had a similar effect on the usages of all three processors in the system, as can be seen from Figure 4-1. All processor usages climb linearly towards their normal values at full load.

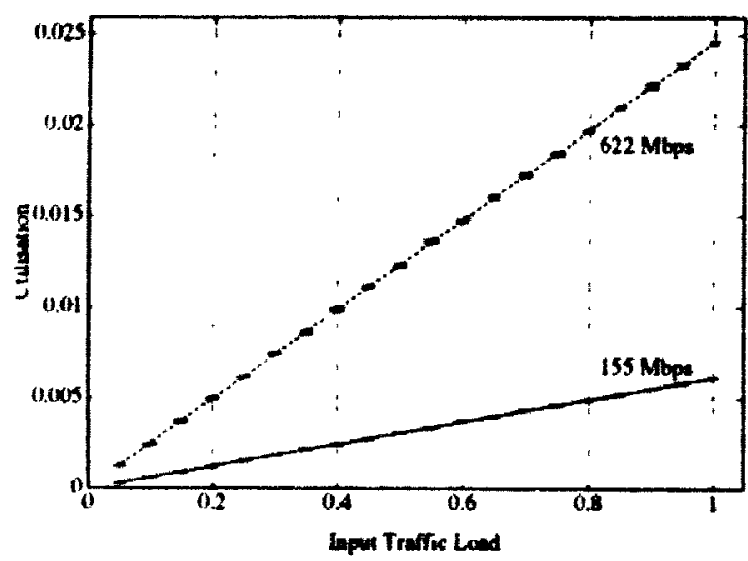

a) MRP Processor Utilisation

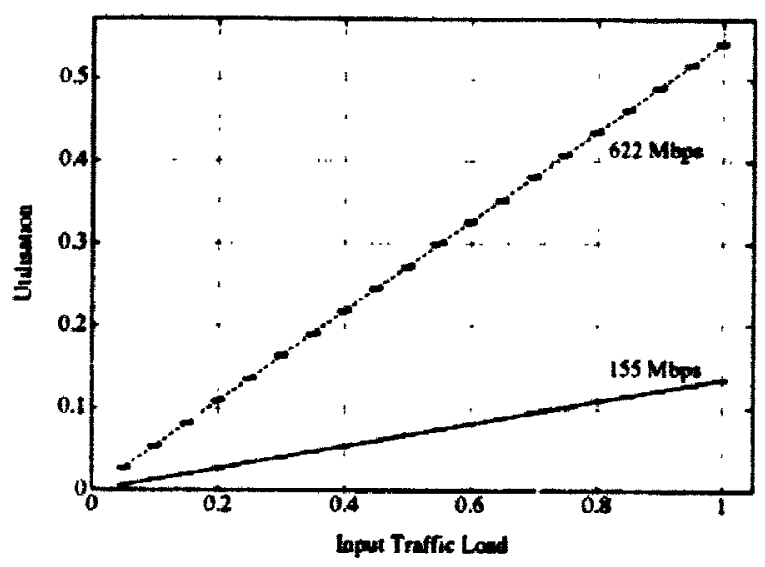

b) RP Processor Utilisation

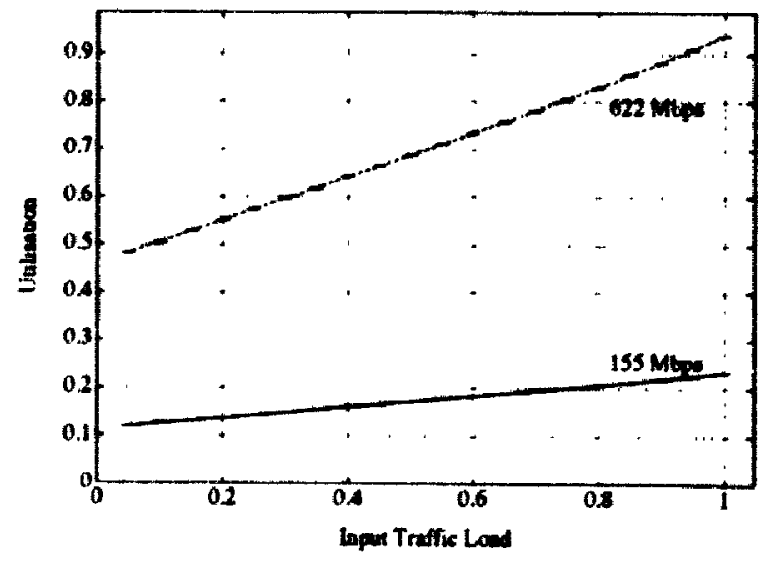

c) BCP Processor Utilisation

Fyure 41 Variation of processor usage with taffic load. 
Note that the BCP processor usage does not go down wero with zero trafici, but rather begins at a value determined by the connection processing time. This minimum vaiue is the ratio of the time taken to process all the connections to the time taken to transmit one scan load of cells. The second of these quantities depends on the cell rate, hence the starting point is greater at 622 Mbps.

The increased amount of traffic results in a corresponding increase in the mean input queue length, as can be seen from Figure 4-2. Although the mean input queue length varies linearly with the traffic load and is affected quite strongly by the bit rate, its value is much lower than one, which indicates that there is not often more than ore cell in the queue. This is because the MRP service time is always less than one cell time at $155 \mathrm{Mbps}$, and less for COM and EOM cells at $622 \mathrm{Mbps.}$

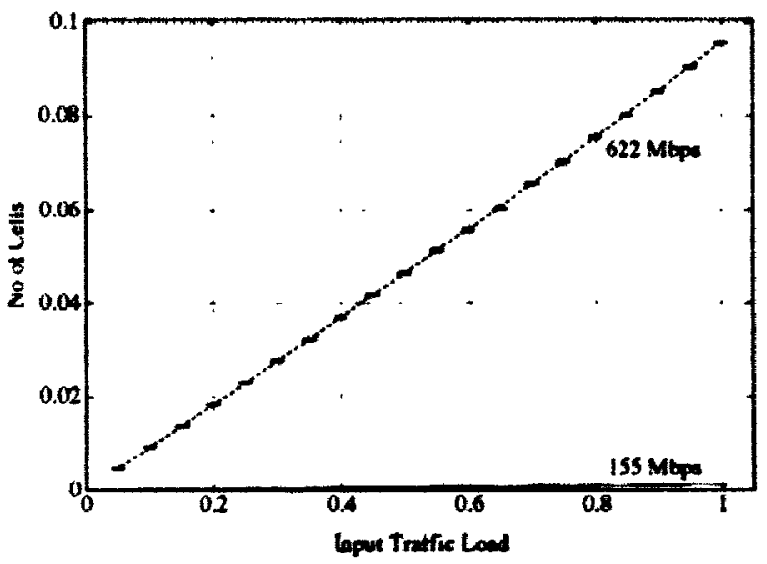

a) Average Input Queve Length

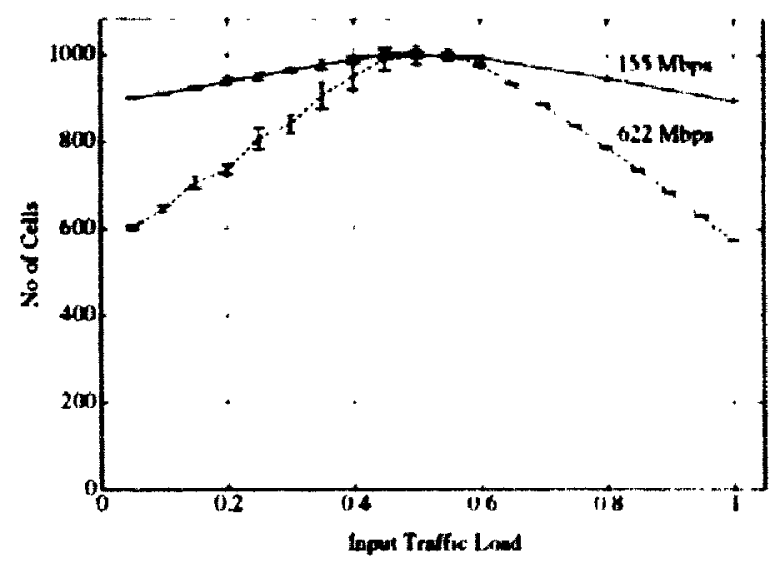

b) Average Output Oueue Length

Figure 4-2 Variation of Inpur and Output Queue lengths with traffic load.

The output queue on the other hand has an average length measured in the hundreds of cells. This is due to the fact that the BCP places cells into the queue in batches, while they are removed from it at a constant rate by the OCU. The shape of the curve is due to the complex way in which the time of entry of the batch of cells into the queue depends upon the instantaneous amount of data in the system (i.e. the BCP processing overhead).

The increasing number of packets flowing into the system had a corresponding effect on the lengths of the Quality of Service queues, as can be seen in Figure 4-3. Note that the 
effect of the RP algorithm is to increase the mean lengths of lower priority queues at higher loads. However, the lengths are still much less than one, so most packets get removed from the queue straight away and there is no difference in the packet delays for different values of QOS.

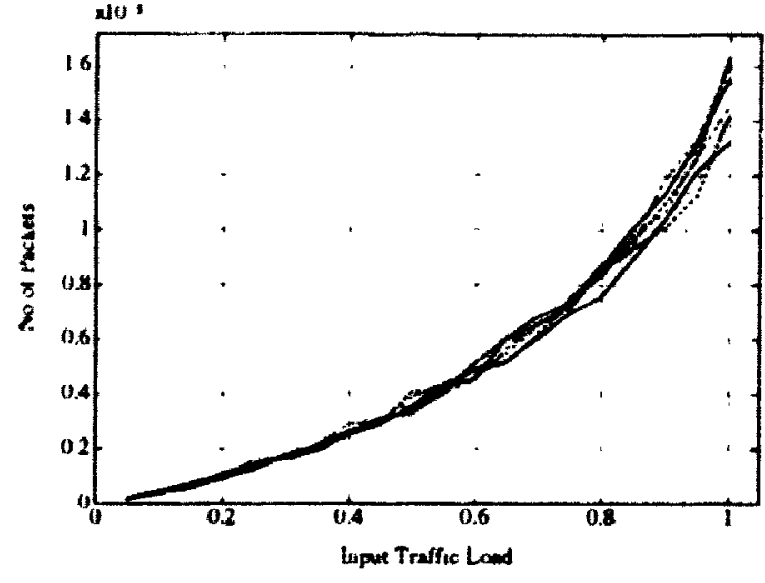

a) Average QOS Queve Lengths at $155 \mathrm{Mbps}$

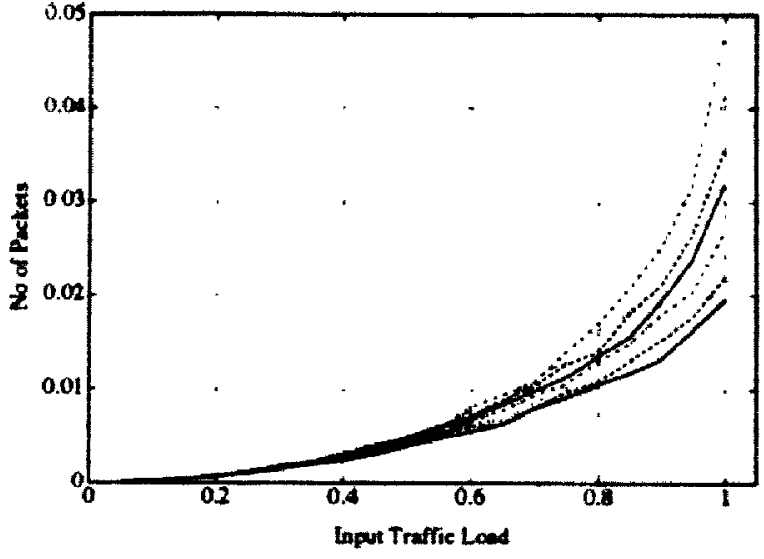

b) Average QOS Queve Lengths at $622 \mathrm{Mbps}$

Figure 4-3 Variation of Quality of Service Queve lengths with traffic load.

The average lengths of each of the outgoing connection queues increase with traffic load in the manner shown in Figure 4-4. The average number of cells in both the Cell and Header RAMs exhibit the same type of behavior, indicating that the majority of cells in the system at any one time belong to packets waiting in the connection queues. Both the connection queues and the RAM contents are the same at the two link rates.

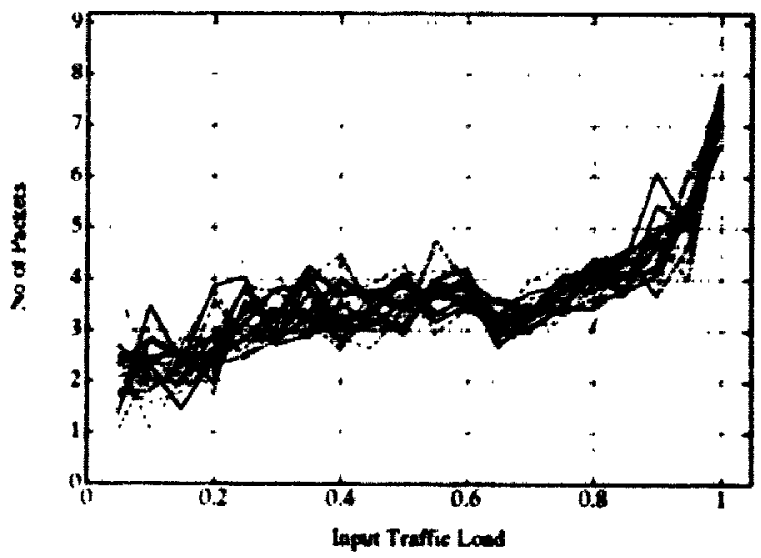

a) Average Connection Queue Lengths at 155 Mbps

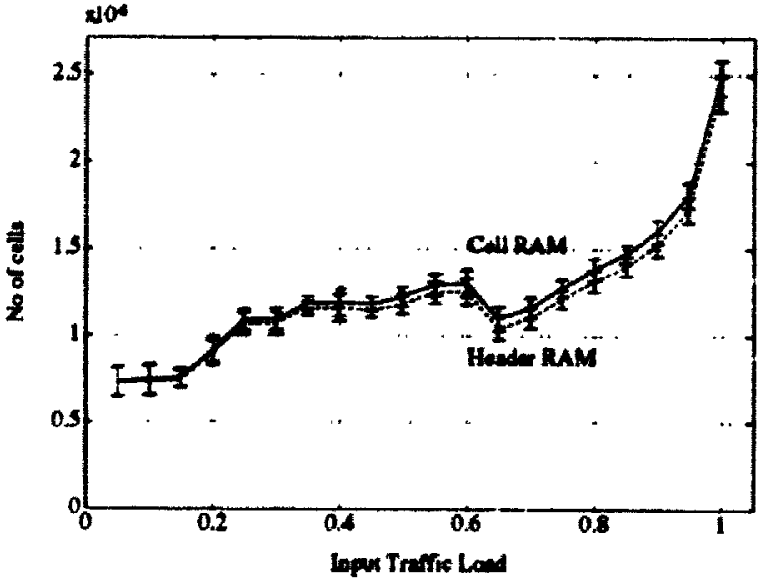

b) Average RAM contents a $155 \mathrm{Mbps}$

Pigure 4 Variation of connection queves and RAM contents with trafic load. 
The average time spert by packets in the system actually decreases with increased traffic load. as Figure 4-5 shows. This effect is due to the fact that the outgoing bandwidth allocations are related to the traffic load. At higher bandwidths the connection queues are served faster, meaning that packets spend less time in the queues despite the higher queue lengths. The majority of the total delay seen by a packet is spent waiting in the connection queue.

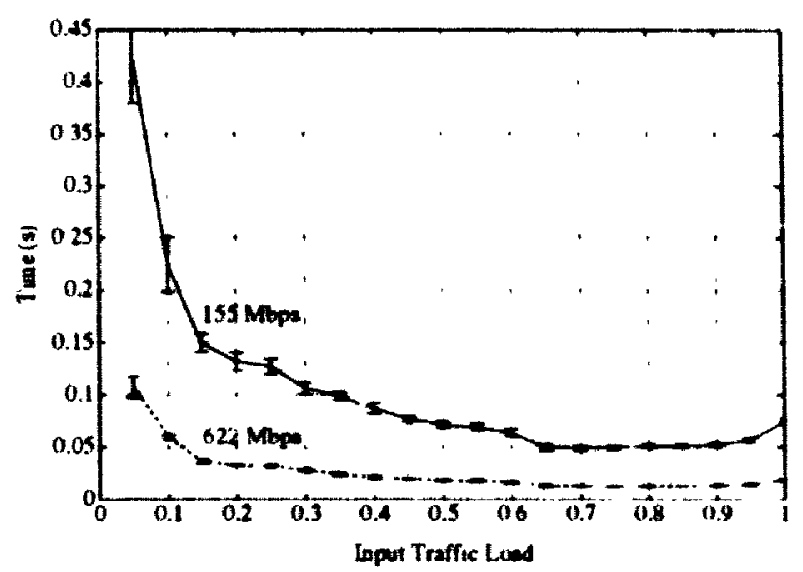

Average Packet Delay

Figure 4-5 Variation of Packet delay with traffic kad

\subsubsection{Burst Length.}

Because little is known about the nature of the traftic in future broadband networks, by necessity one of the most arbitrary assumptions made about the input traffic for this experiment was the average burst length. The simulation results revealed that this parameter can have a significant offect on the system. The problem relates to the fact that at a mean burst length close to the miximum packet size, a high proportion of bursts are shorter than one full packet. This reduces the mean packet length. resulting in an increase in the rate of incoming packets to the system at a given traffic load. The increased packet rate causes a higher load on the MRP and RP processors, as illustrated by Figure 4-6.

At 155 Mbps there is no problem, but at 622 Mbps the Routing Processor is running at full capacity for mean burst lengths below 100 cells (4.4 kilobytes). This implies that the RP is no longer able to service packets at the rate that they are coming in. In fact, the mea- 


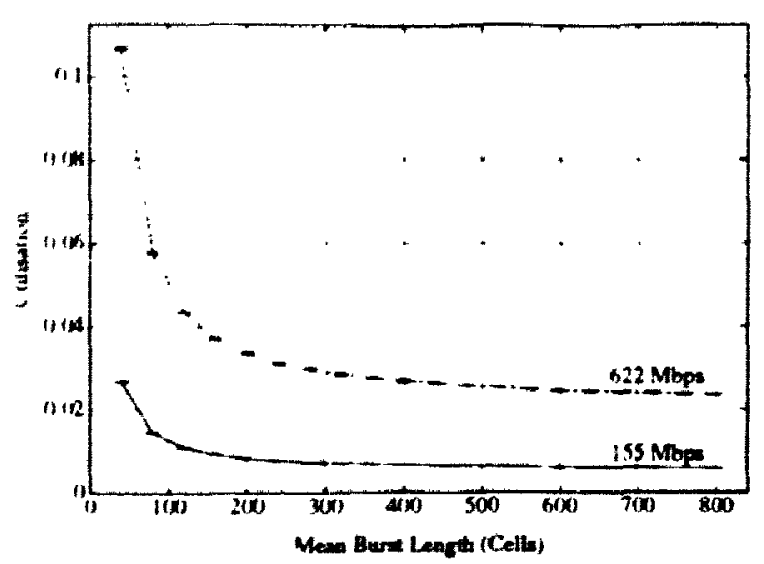

a) MRP Processor Utilisation

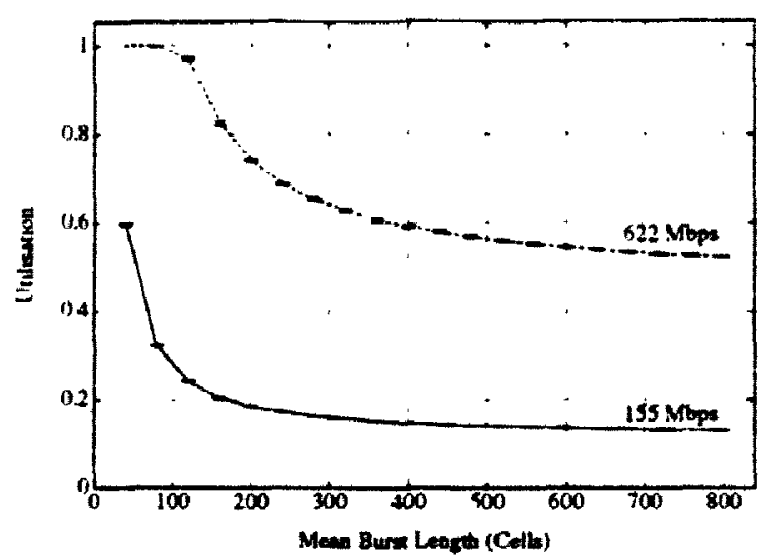

b) RP Processor Utilisation

Figure 4-6 Effect of Burst Length on MRP and RP Load.

sured output traffic load drops below the input at these points, indicating that traffic is building up in the system.

The result is that the system becomes unstable. The sizes of the Cell and Header RAM contents grow without bound while the packet delay increases sharply, as shown in Figure 4-7. Note that as the burst length decreases towards the failure point, the packet delay is seen to decrease - this is because as discussed earlier the measured packet delay depends on the mean packet length, which starts to decrease as the proportion of unfilled packets increases.

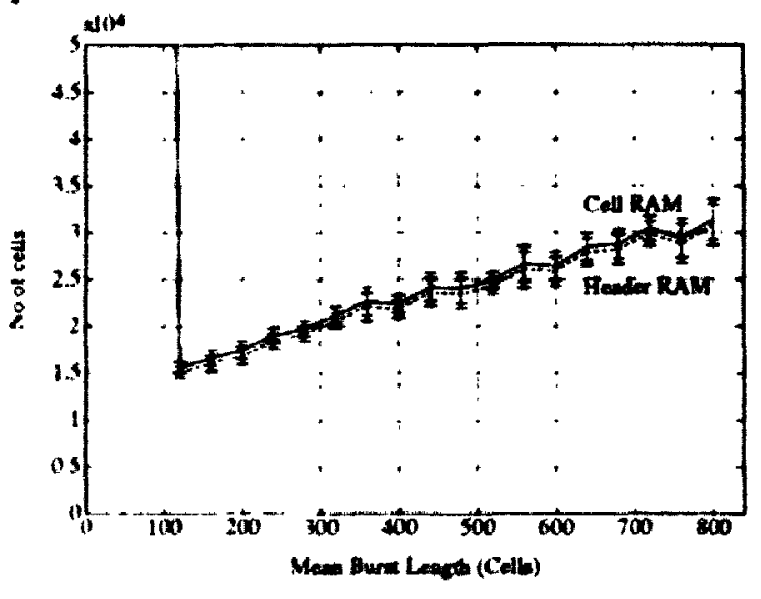

a) Average Cell and Header RAM Contents at $622 \mathrm{Mbps}$

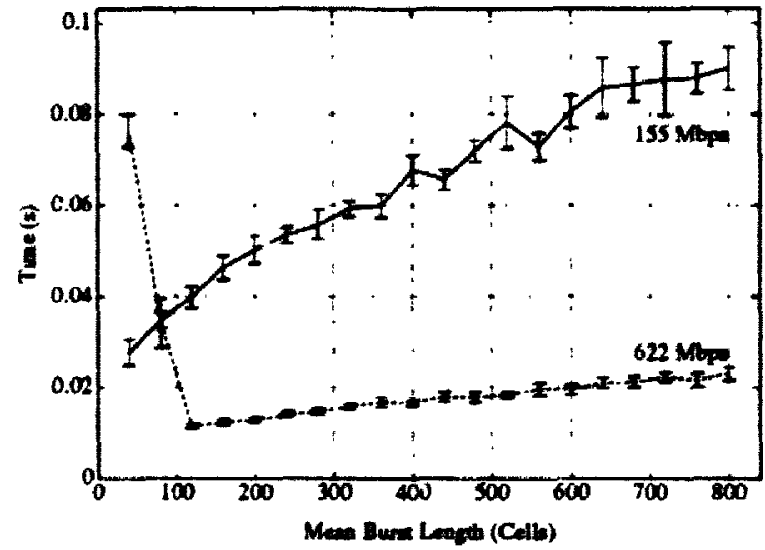

b) Average Packet Delay

Figure 47 Variation of system contents and packet delays with burst length. 
The point at which the system overloads depends on both the RP processing speed and the traffic load. If it is known that the mean burst length in a system is likely to he less than the breakpoint for a given RP speed, then it may be necessary to add an extra processor to the RP to allow it to continue to operate at full load. However, it seems unlikely that future broadband networks would carry traffic with such a low mean burst length. as the bulk of the traffic is likely to be file and image transfers.

\section{4,4.1.3 Cell Error Rate.}

The rate at which cells arrive with errors in them (including bit errors, dropped or misinserted cells and ARORT cells) was varied from one cell in 7 million to one cell in 100. Errored cells are dropped by the ICU, while the rest of the packet is removed from the system by the MRP, including cells already in the system and cells still to arrive. The result is a reduction in the output load of the system and an increase in MRP usage, as Figure 4-8 shows.

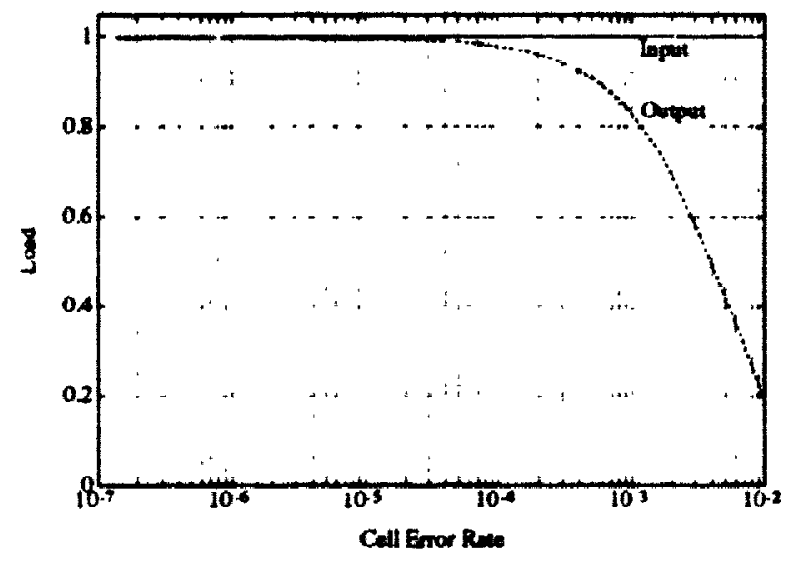

a) Input and Output Link Utilisations

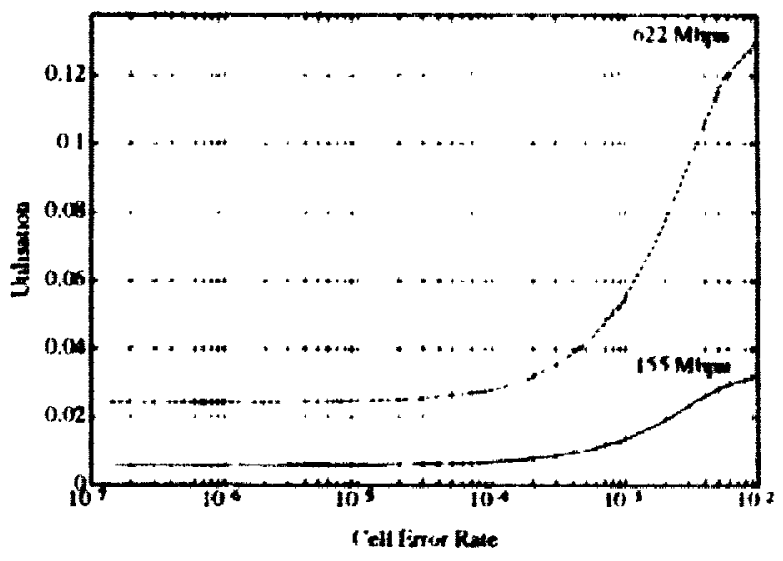

b) MRP Processor Utilisation at $155 \mathrm{Mbps}$

Figure 4-8 Effect of cell enror rate on traffic and MRP usage.

Below a cell error rate of one in 10000 , the effect on the system is small. As this rate increases, though, the amount of traffic in the system stans to drop. The QOS queues are not affected and neither is the RP load since in most cases the MRP cancels the packet while the RP is processing it. The result is a drop in the connection queue lengths, causing a decrease in the RAM contents and a drop in the BCP usage. 
Chapter 4: Experimental Results.

Plotting the observed output load against the BCP usage gives an identical plot to that seen in Figure 4-1, showing that the BCP usage corresponds to the actual outgoing traffic. The packet delay and RAM contents however are lower. since the outgoing bandwidth allowances are now greater than the actual outgoing traffic. Compare the plots in Figure 49 to those in Figures 4-4 and 4-5.

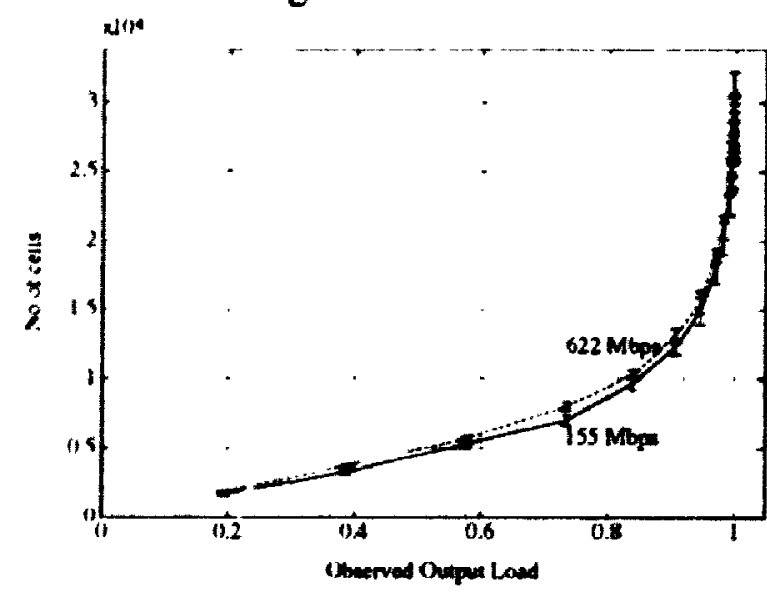

a) Cell RAM contents

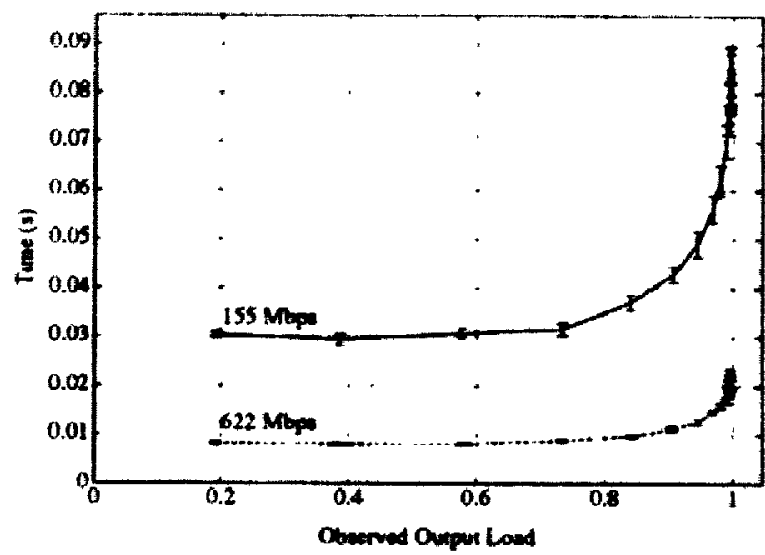

b) Average Packet Delay

Figure 4-9 Effect of changing output loed due to cell error " se.

This illustrates that the most significant restriction on system performance is the requirement that the outgoing traffic adhere to the agreed traffic parameters. When the bandwidth allocation is greater than the actual traffic, then the data may be retransmitted soon after it arrives and the packet delays and amount of backlog in the system are reduced.

Note that in the real system, the BCP would interpret the decreased traffic as requiring a decrease in the bandwidth allocations for the connections. Once the message had been sent to the ATM control and the bandwidth reduced, the BCP usage and packet delays would return to the values corresponding to the actual output traffic level.

\subsubsection{Group Address Probability.}

Increasing the probability of a burst having multiple destinations has the opposite effect on the system to the error rate. Instead of the output traffic load decreasing, the load 
increases as traffic multiplias within the system. To see this effect, a simulation was made with an input traffic load of $50 \%$.

Because the model does not include a variable output bandwidth scheme, the increased output traffic can only be transmitted at ine input rate. The BCP still empties out the connection queues at a tixed rate corresponding to the circuit bandwidth settings, but the rate at which packets are entering the queues has increased due to the packets multiplying. The result is that once the actual output load begins to increase, packets start to huild up in the system and the system becomes unstable. In the real situation the BCP would quickly detect this and request an increase in the output bandwidths to a value appropriate to the observed output traffic.

It is worth noting that group addressing only stars having a significant effect on the system when the group address probability approaches $10^{-1}$. or 1 in 10 messages. and that any bandwidth adjusting algorithms would be quite capable of nullifying the effects. Thus, group addressing is not a significant concern to the system.

\subsubsection{Effect of the MRP model parameters.}

There is only one assumed parameter of the Message Reassembly Processor model that is likely to have a significant effect on the system, that of the BOM cell processing time, so only a single simulation was made under this category.

The purpose of the simulation was to determine the effects on the system of altering the MRP BOM time, so as to get an idea as to whether any inaccuracies introduced by an incorrect assumption are likely to be significant. It also determined the maximum value of this processing time, beyond which the system fails to operate.

The default value assumed for the MRP BOM time was such that the MRP processor usage was very low. To have an effect on the system, it was necessary to push the processing time up to a similar scale to the average rate of incoming packets. Thus, the range of 
values used in the simulation was chosen so as to force the MRP processor usage up to a high value. The result of course is to cause the system to fail, as is evidenced by the output loads in Figure 4-10a.

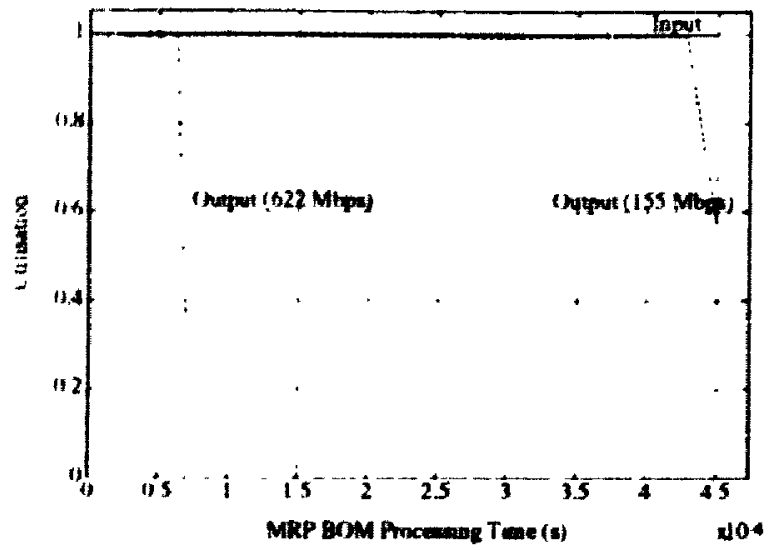

a) Input and Output link utilisation.

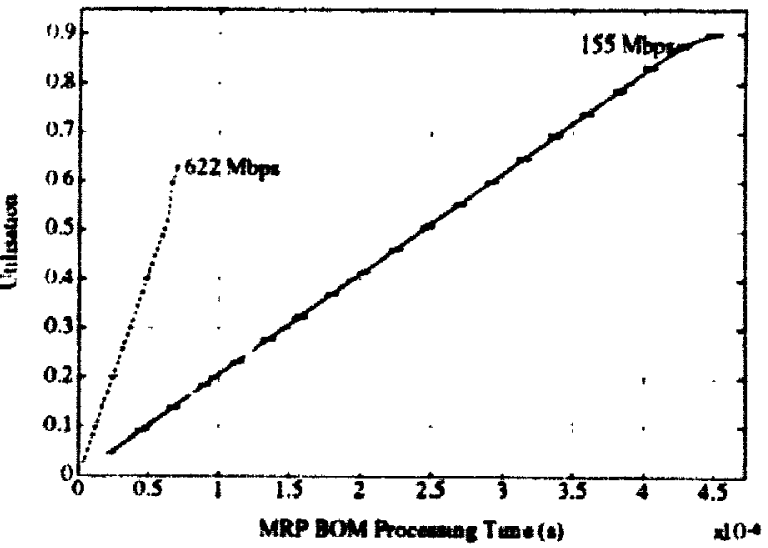

b) MRP Processor Utilisation

Figure 4-10 Result of increasing MRP BOM time on link loading and MRP usage.

Figure 4-10b illustrates that the system failure occurs before the MRP processor reaches full utilisation. This is because the point of failure is when the average MRP processing time per packet exceeds the incoming packet rate. The average processing time includes the hardware COM and EOM times, which are not included in the processor usage measurements.

From these results, the maximum values of the MRP BOM processing time permissible before the system will fail are as given in Table 4-7. It should be noted that these values are at least an order of magnitude greater than the estimate used as a default. Thus, it is unlikely that the MRP processing time would be a critical issue in the CLSF design.

\begin{tabular}{|l|l|}
\hline \multicolumn{1}{|c|}{ Link Rnte } & MRP BOM Tive Limit \\
\hline $155 \mathrm{Mbps}$ & $420 \mu \mathrm{s}$ \\
\hline $622 \mathrm{Mbps}$ & $60 \mu \mathrm{s}$ \\
\hline
\end{tabular}

Table 47 Processing Limits for the MRP

The presence of a limit on the input queue size has the effect of preventing data from building up in the system when the MRP BOM time is too great. Cells that arrive when the 
queue is full are simply discarded. which generates a sequence error and causes the MRP to remove the rest of the packet from the system also. Hence above the breakpoint the number of cells in the header KAM drops towards zero as more and more packets are removed because of sequence errors, and the number of cells in the cell RAM drops towards the input queue size.

Below the point of failure, none of the other system statistics show any variation due to the changes in processing time. From this it can be concluded that the system performance is not dependent on the MRP BOM time.

\subsubsection{Effect of the RP model parameters.}

As with the MRP case, there is only one important parameter in the Routing Processor. that of the packet processing time, and so only one simulation was required. Again. the purpose of the simulation was to determine the maximum value of the processing time before system failure, and whether or not any errors introduced by the choice of defaults are important.

The value of the RP processing time was increased to the point where system failure occurred, as shown in Figure 4-11.

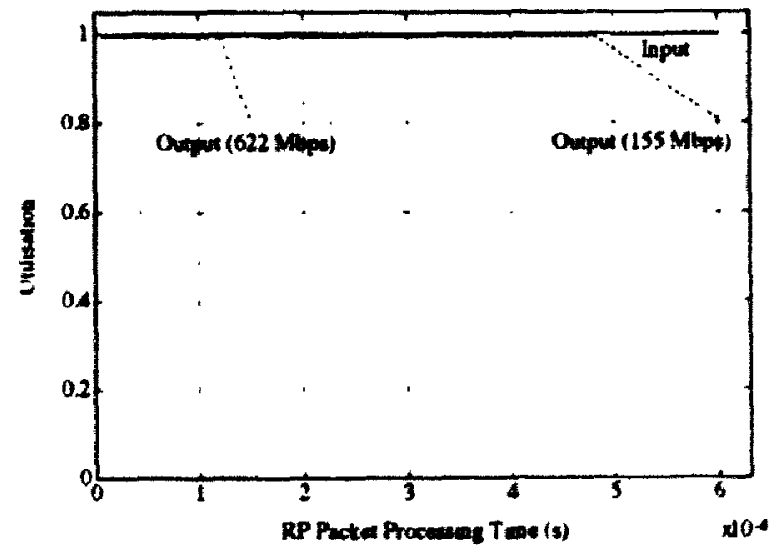

a) Average Link Utilisation

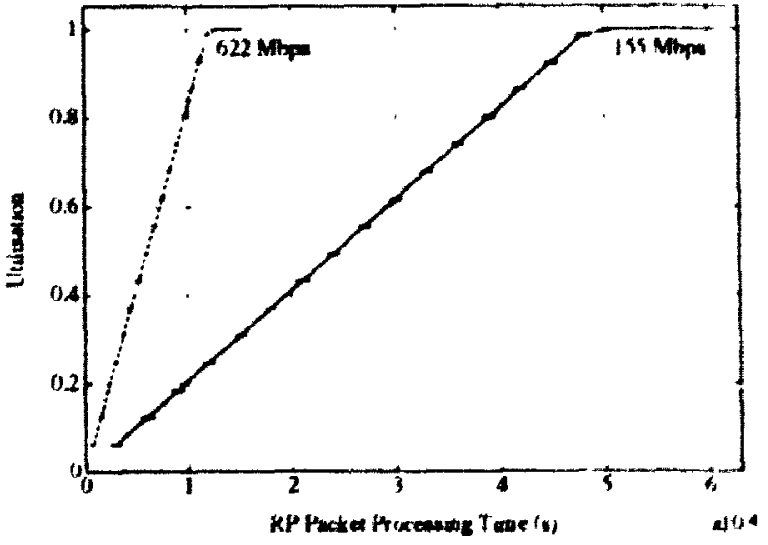

b) Average RP Utilisation.

Figure 4-11 Effect of RP processing time on link traffic and RP utilisation. 
From these results the limits presented in Table 4-8 can be determined for the RP processing time.

\begin{tabular}{|l|l|}
\hline \multicolumn{1}{|c|}{ Link Rate } & Max. RP Processing Time \\
\hline $155 \mathrm{Mbps}$ & $480 \mu \mathrm{s}$ \\
\hline $622 \mathrm{Mbps}$ & $120 \mu \mathrm{s}$ \\
\hline
\end{tabular}

Table 4-8 Limits on RP Processing Time.

At $155 \mathrm{Mbps}$ the maximum processing time is much higher than the estimate used for the default, but at $622 \mathrm{Mbps}$ it is only twice the default value. This means that it is quite possible that the execution time of the RP algorithm could end up being too great for system operation at $622 \mathrm{Mbps}$. As was shown in Section 4.4.1.2, the problem is made worse when the mean burst length is decreased.

Given a fast processor ind careful programming, though, there is no reason why the RP execution time could not be made to fit within the limits. The maximum processing tin e at $622 \mathrm{Mbps}$ is still 3600 machine cycles at $30 \mathrm{Mhz}$, which would allow an algorithm of at least $\mathbf{1 8 0 0}$ sequential instructions to execute. This figure can be increased if instruction pre-fetching is used in the processor, or if a faster clock is used.

None of the other system statistics were affected by the RP processing time below the breakpoint, with the exception of the QOS queue lengths which exhibited the same type of spreading out observed in Figure 4-3. The packet delays and number of cells in the RAM were not affected because these de? " d primarily on the BCP transmission rate rather than the RP time. Again, it can be concluded that under normal conditions the RP processing time has no significant effect on the system.

\subsubsection{Effect of the BCP model parameters.}

Of the Bandwidth Control Processor parameters, the most arbitrary default parameter value was the connection processing time. As with the RP and MRP experiments, the 
value of this parameter was altered to see what effect it has on the system. and what are the limits within which the BCP software must operate.

The results of the simulation show the point of system failure in Figure 4-12.

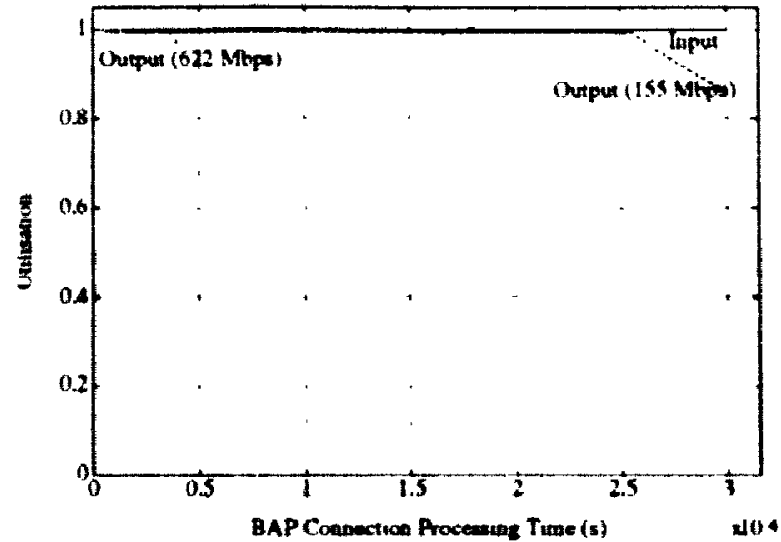

a) Average Link Utilisation

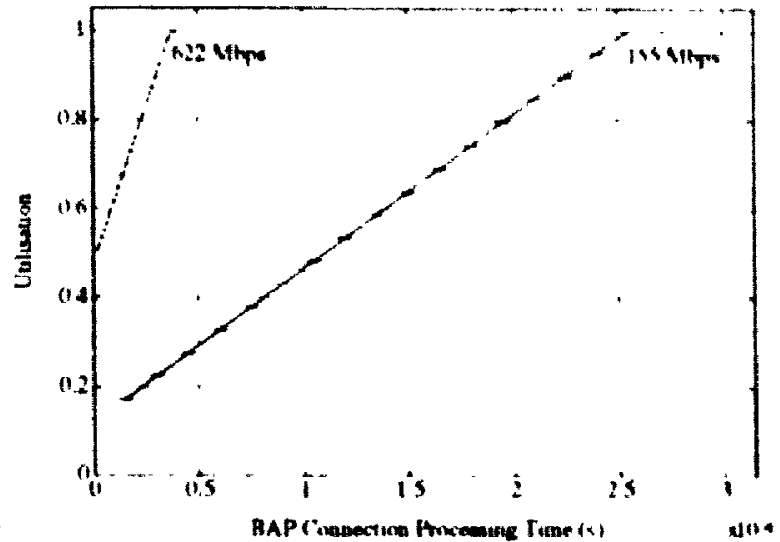

b) Average BCP Utilisation.

Figure 4-12 Effect of BCP processing time on output traffic and BCP utilisation

The BCP processing time did not affect either the number of cells in the system or the packet delay below the point of failure. This is an indication that the algorithm is working as expected, since the sel vice rate of the connection queues does not depend on the BCP algorithm execution speed, but rather on the bandwidth settings.

The simulation revealed the algorithm operating limits given the other default parameter settings. It is important to note that the locations of the breakpoints depend also on the number of connections, the cell copy time and the number of cells per scan of the BCP algorithm. The first two are a property of the system environment, but the latter is a property of the algorithm that may be used to compensate for the BCP processing time.

To see the effect of this type of compensation, the number of cells per BCP scan was varied. The results showed that an excessively high BCP processor usage can be reduced to a certain extent by adjusting the algorithm. Figure 4-13 shows the effect that this has on the BCP utilisation and on the output queue. 


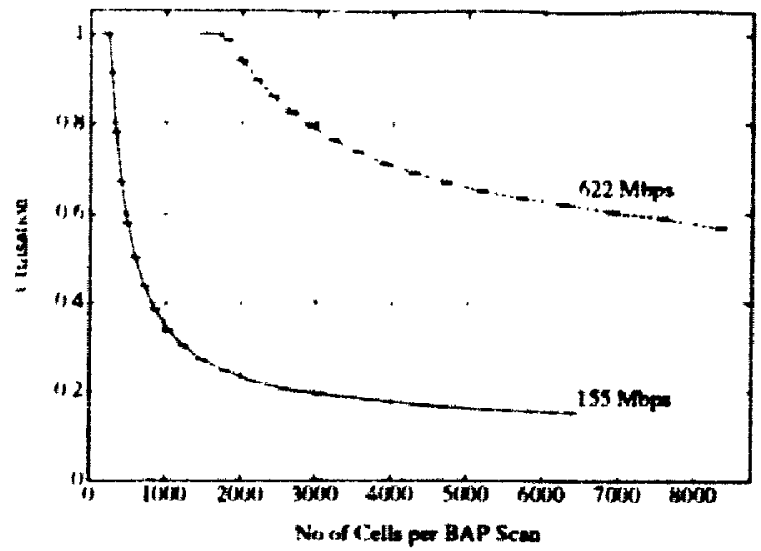

a) Average BCP Utilisation.

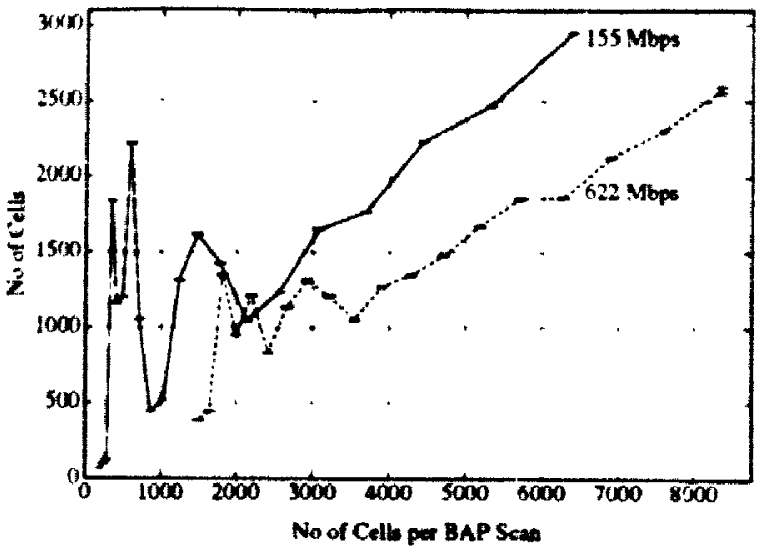

b) Average Output Queve Length.

Figure 4-13 Effect of BCP scan algorithm on processor usage and output queue.

The output queue again exhibits complex behavior due to the manner in which the BAP tills it in bursts. This variation in mean queue length is not significant, though, as the instantaneous length is continually varying from near empty to the scan size. The cells continue to be transmitted at a constant rate.

The output link load results of this simulation confirm that the number of cells per scan multiplied by the cell transmission time must be higher than the total BCP processing time per scan, or else the system will fail. The conditions within which the parameters must lie for proper system operation are given in Table 4-9.

\begin{tabular}{|l|l|l|}
\hline \multicolumn{1}{|c|}{ Limk Rate } & \multicolumn{1}{c|}{ Operating Conditions } & \multicolumn{1}{c|}{ Key to symbols } \\
\hline \hline 155Mhps & $\frac{C T_{p}}{S}+T_{c}<2.74 \times 10^{-6}$ & $\begin{array}{l}T_{p}=\text { Connection processing time } \\
T_{c}=\text { Cell copy time }\end{array}$ \\
\hline $622 \mathrm{Mbps}$ & $\frac{C T_{p}}{S}+T_{c}<6.82 \times 10^{-7}$ & $\begin{array}{l}S=\text { No of cells per scan } \\
C=\text { No of connections. }\end{array}$ \\
\hline
\end{tabular}

Table 4-9 Operating Limits on BCP algorithm.

It can be seen from these equations that if $T_{c}$ is too large, then there will be no realistic value of $S$ that can satisfy the conditions. Note also that the maximum output queue size depends on $S$. If $S$ is too high. then the required output queue may be impractical. This factor also tends to put a restraint on $T_{p}$. 
Both the packet delay and number of cells in the cell RAM are dependent on the scan length, as illustrated in Figure 4-14. This dependence comes about beciause as the scan size increases, the time between scans increases, and so packets spend more time waiting in the connection queues resulting in higher RAM sizes.

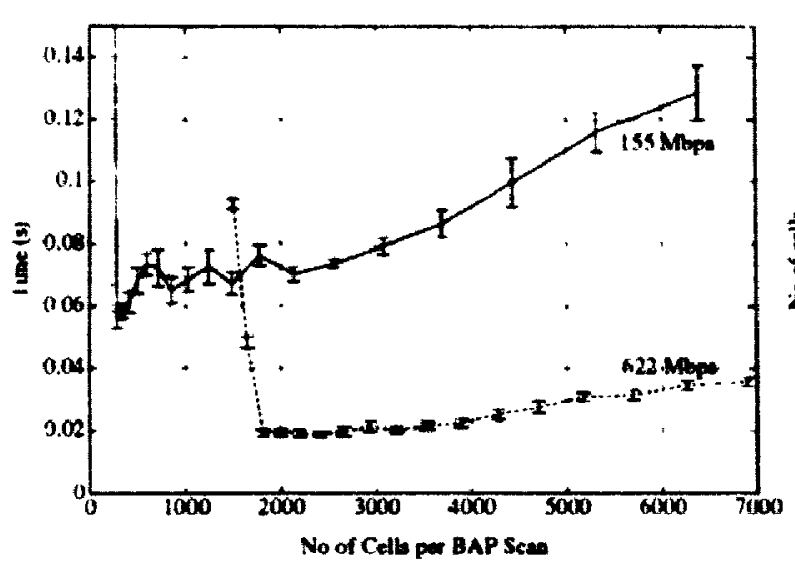

a) Average Packet Delay.

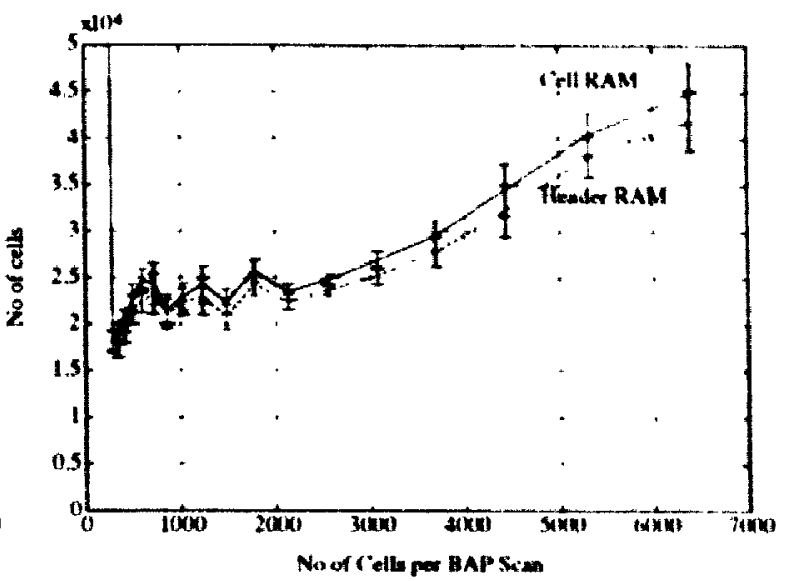

b) Average System Contents a 155 Mhps.

Figure 4-14 Effect of BCP scan size on packet delays and cells in system.

Because the BCP utilisation and the point of failure depend on the number of active connections, it would be desirable to modify the BCP algorithm so as to dynamically adjust the scan length to compensate for changes in the number of connections. In this way the BCP utilisation and point of failure could be controlled. The only drawback is the effect that the scan size has on the system performance.

\subsection{Conclusions.}

It is apparent from observing the trends in the results that so long as the processing times within the system fall within certain limits, they have little effect on the performance of the system as a whole. The only parameters that significantly affect the system are those related to the traffic passing through it.

At full traffic load, the processor usages were observed to be significantly less than one for both link rates, implying that some hardware savings may be achieved by combining processors together. This possibility is elaborated upon in the next chapter. 
The mean burst length of the input traffic can cause system failure if it falls too low. This however is unlikely to be a problem in a broadband network as most data or file transfers will involve bursts significantly longer than the packet length.

The limits on the BCP processing time and its usage can be controlled to a certain extent by varying the number of cells per scan of the algorithm. This can be done dynamically in response to a changing system environment.

The factor most relevant to system performance is the output bandwidth allocation. The CLSF can be seen as a "black box", into which bursty traffic is going on one virtual connection, is being switched in a random fashion to another virtual connection, and is leaving at a fixed average rate. Because of the random nature of the switching, bursts of traffic on to one or another of the virtual connections tend to occur and they take a long time to clear at the mean rate. The result is that data has a tendency to build up in the system to the extent that it is the queueing delay at the output, and not the system processing, that affects the quality of service. This applies regardless of the system design.

In order to reduce the delays, it would be necessary to increase the rate at which traffic leaves the system. This implies a bandwidth allowance that is slightly higher than the actual traffic level. By using a bandwidth adjusting scheme that allows a slight amount of unused capacity, the amount of data in the system and the quality of service seen by that data could be much more easily regulated.

Regarding this design in particular, it can be concluded from the results that the design as it stands is capable of handling a full traffic load at both $155 \mathrm{Mbps}$ and $622 \mathrm{Mbps}$, provided that the software execution times are within the prescribed limits. In all cases the default times used were generous, so it is quite possible that a real system could be constructed that would fit within those limits. It can therefore be stated that this design poses a valid solution to the tasks required of a BISDN connectionless server. 


\section{Chapter 5: Modifications to the Design.}

\subsection{Processor Configurations.}

An examination of the simulation results reveals that using the default settings neither the MRP nor the RP are fully utilised at $622 \mathrm{Mbps}$, and none of the processors are being used to capacity at 155 Mbps. The MRP processor in particular is only used less than $2.5 \%$ of the time at $622 \mathrm{Mbps}$. This implies that some hardware savings could be achieved by combining processors together.

For example, if the system is running at $155 \mathrm{Mbps}$ and the actual processing times were the same as those used as defaults, then the maximum MRP usage at full load is less than $1 \%$, the RP less than 15\%, and the BCP less than 25\%. A multi-tasking processor could be doing the job of all thice and still only be used on average $41 \%$ of the time.

The hardware requirements would then be much simpler. The Header RAM need not be dual port, and only a single data bus, address bus and system queue are required. The processor would split its processing time between the BCP and the RP tasks, and would service the MRP tasks only when interrupted by the MRP hardware.

The only disadvantage of combining the processors in this way is that the average processing times of each job would be higher than in the present situation, because the processor would not always be able to answer interrupts immediately or work exclusively on one packet or connection. However, the simulations revealed that it is the outgoing bandwidths and not the processing speed that affect the quality of service seen by the data traffic, and so the extra delays would not affect the performance of the server.

At 622 Mbps the BCP processor is running at near full capacity, so combining it with the other two units would not be possible. A two processor configuration would be necessary, with one processor looking after both the MRP and the RP, and the other responsible 
Chapter 5: Modifications to the Design.

only for the BCP. A dual port header RAM may well be necessary due to the frequency of memory access by the BCP processor algorithm.

Whether or not the processors can be combined would depend on the exact processing times required for each task, which in turn depend on the particular processor chosen and the coding of the algorithms. In designing a real system it would be necessary to first code the software, then run the simulation program to get an idea of the processor usage, before deciding whether it is feasible to combine processors.

\subsection{Increasing System Capacity.}

The capacity of the CLSF could be increased by adding extra physical links to the system. This could be achieved by duplicating the current design, in a manner similar to the initial design approach described in Section 3.1.2 on page 33.

Each incoming/outgoing link pair would have its own ICU, Cell RAM, OCU, MRP hardware. Header RAM and BCP hardware. The processors, on the other hand, could be split among the links instead of among the modules as in the previous section. The number of links serviced per processor will depend on the traffic parameters and the software execution times, and should be chosen so that the average processor usages are less than one. The System Manager would also be common to all the links.

In order to avoid memory and bus conflicts, each header RAM should only be served by at most one of each of the MRP, RP and BCP processors. This means that the number of links that a processor can service is determined by the inverse of its processor usage rounded down to the nearest integer. For example, given the default parameters and results observed in the simulation experiment, at 155 Mbps the MRP processor could service about 140 links. the RP could service 6 links and the BCP could service 4 . More practically, the RP would be combined with the MRP as described above. 
At $622 \mathrm{Mbps}$, both the RP and BCP usages are greater than 0.5 so no combination of processors across the links is possible.

In order to spread the load across all the CLSF modules in a multi-link system, a subset of the terminals would need to be connected to each server. However, a conseyuence of the current design is that no switching of data between different physical links is possible there is no way that cell data stored in one cell RAM could be output on the physical link connected to another. If a packet comes in on one link destined for a terminal connected to another, then it is necessary to use the underlying ATM network to aid in the routing of the data.

One way in which this could be achieved is to treat each module as a separate CLSF entity, and to use trunk connections through the local ATM switch to route data between them. Packets would enter the input of one CLSF module and be switched onto a trunk connection, which takes them from the output of that module through the ATM switch to the input of another, then be switched by the second module on to their destination circuit. This effectively doubles the delay seen by the packet, as well as resulting in extra load on the ATM/CLSF system.

A more practical alternative is to take advantage of the fact that the virtual circuit resource is almost unlimited, and simply use multiple outgoing virtual circuit connections. Each terminal is connected to the input of only one of the CLSF modules, but there exists a one-way connection from the outputs of all the CLSF modules to the terminal. This arrangement is illustrated in Figure 5-1.

In this way the load on the modules can still be controlled by directing the traffic from a particular terminal to a given module, but packets may be switched to any of the local terminals in one step. The only drawback is that each BCP now has to maintain output queues for all terminals, not just the ones connected to its own module. This could result in significantly higher processing loads in times of congestion. 


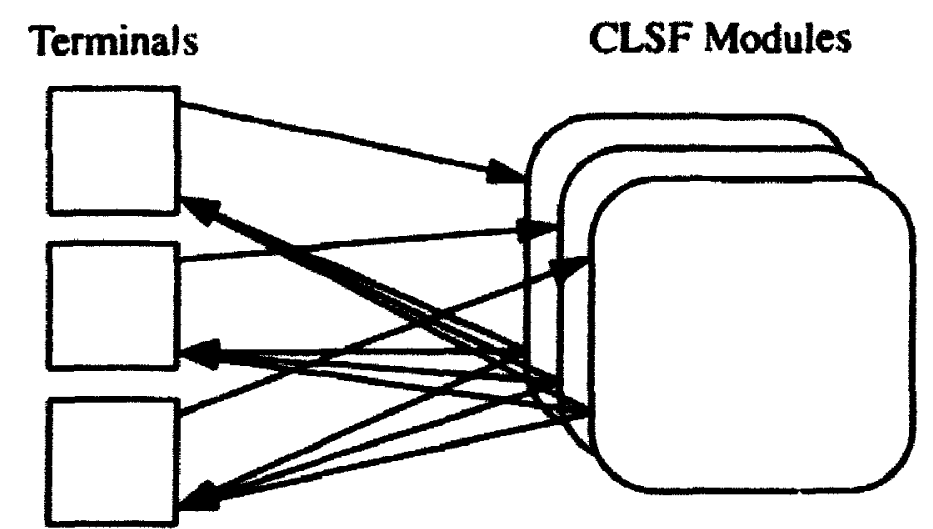

Magure 5-1 Arrangemem of virtual circuits for a multilink systom

The best arrangement would probably be to use a combination of the above two approaches, by dividing the modules up into groups. Packets switched between modules of a particular group use the multiple virtual circuit approach, while packets switched between groups are processed twice. Care would need to be taken to ensure that terminals that are likely to have a high rate of traffic between them (e.g terminals all belonging to the same company) are connected to modules of the same group.

In this way the processing load on the system is minimised, with double processing only necessary on low usage routes, but the potential load on the BCPs is also kept low to prevent system failure. How exactly a particular system is organised will depend heavily on the local environment.

\subsection{Use of an alternative AAL Layer.}

There exists a recent proposal within the CCITT to provide a fifth AAL protocol for use with both connectionless and connection oriented data ${ }^{[46]}$. The new AAL is a greatly simplified version of the current standards, where there is no SAR layer protocol overhead and no CS layer header. The result is that all 48 octets of the cell payload are available for user data, resulting in improved transfer speed and efficiency.

The lack of SAR overhead means that multiplexing at the SAR layer is not supported, since there is no MID field. This means that there can only be one data connection on each 
virtual circuit, and only one packet transmitted at a time. This is not a handicap for two reasons, firstly because the available bandwidth on a given virtual circuit remains the same, so that two consecutive packets will be transmitted in exactly the same time as two multiplexed ones under the current system. Secondly, the virtual circuit resource is so huge that removing the capability for an additional layer of connectivity does not cause any problems. If it is desired to have multiple connections to a particular destination, then they may be easily be grouped together using virtual paths.

The current proposal is that the new AAL be adopled for all connection oriented data. and for connectionless data that uses the indirect connectionless service. For compatibility with already installed equipment (DQDB MANs), connectionless data that utilises the direct connectionless service (i.e. all CLSF traffic) will continue to use the existing standard. There is strong support for this new AAL, and it may have already become a part of the BISDN definition.

This means that it is not currently intended for the CLSF to switch data that is using the new protocol. In fact, with some minor modifications to the hardware design and the processor sofiware, this CLSF architecture could handle both types of traffic at once.

Firstly, the ICU and OCU should both be modified so that the entire 48-bit cell payload is stored in the cell RAM instead of the current 44. The ICU still separates the cell headers in the same way as at present, but the SAR word gc $;$ into the cell RAM as well as the outgoing header queue. The OCU must be capable of restoring the four octets corresponding to the SAR information of cells that use the new AAL.

For each virtual connection, it should be specified at setup time which of the two AAL types is to be used. This information will be stored in the active connection table. When the MRP receives a cell from a simplified AAL connection, instead of interpreting the SAR layer word from the header queve entry it simply ignores it. An indication in the circuit queues will similarly instruct the BCP to use a different algorithm for bandwidth con- 
trol, and to pass an indication into the outgoing header queue to instruct the OCU not to replace the SAR bytes of the outgoing cells.

Because of the difference in cell payload sizes, however, the design as it stands is not rapable of routing data from circuits that use one type of AAL to circuits that use the other. That would require changing the cell boundaries within the data, which is not possible in this system because of the way the data is stored as individual cells in the cell RAM.

Because of its simplicity, an exclusive use of the new AAL would actually greatly simplify this CLSF design. The lack of SAR protocol information means that the MRP hardware need not perform any error checking on the cell data. No SAR layer multiplexing is supported, so the circuit queues and connection queues used by the RP and BCP could be combined, thus simplifying the software algorithms and data structures. The BCP need not split a circuit's bandwidth between high and low priority and multiplexed connections, simplifying its operating algorithm and increasing its processing speed.

Thus, a CLSF designed to use the new AAL would be much simpler, faster and would have greater capacity than the current system. Many of the problems that needed solving during this work would not have occurred. In the opinion of the author, from an engineering standpoint the simplified AAL is a much more practical choice of protocol. The current AAL type 4 is unnecessarily complex for use in a BISDN environment, and introduces capabilities that are not really required.

It is a fact of life, however, that one must design for backwards compatibility. Given that there is already a large installed base of systems that use the DQDB standard (which is nearly identical to the current AAL type 4), it is necessary to make a choice between having a small number of complex CLSFs with a large number of simple DQDB/ATM gateways, or the reverse. The decision involves a lot of factors other than purely engineering ones, and it will no doubt be up to individual administrations to make their own choice based on their particular circumstances. 


\section{Chapter 6: Conclusions.}

In this work, a design for a BISDN connectionless server has been prevented which has been shown by experiment to be capable of operating at the standard link rates of 155 Mbps and 622 Mbps. Its operation conforms with the current detinition of the BISDN and the BISDN connectionless service as detined in the CCITT I series of recommendations. Special purpose hardware circuits have been proposed which perform some of the protocol processing, enabling the system to operate at high speed.

The I series of recommendations specifies that the Broadband Integrated Services Digital Network. based on the connection-oriented Asynchronous Transfer Mode, is capable of carrying connectionless data traffic in two possible ways. The first of these. known as the indirect method. consists of point to point virtual circuit links between pairs of network gateways, and has been shown to be suitable only for a certain limited range of traffic types. The second method, known as the directly supported connectionless service, consists of a logical overlay network of virtual connections and connectionless servers (CLSFs) lying on top of the ATM network.

The job of the CLSF is to route data packets from one virtual connection to another according to information stored within the packet. It does this by examining the destination address that is contained in the first cell of the packet, in the CLNAP layer protocol header, and using that address to select a new outgoing virtual circuit for the packet. It then routes this and all future cells of the packet onto the new circuit by simply translating the VCI number of those cells.

To do this, it must be able to distinguish between cells belonging to different incoming packets by logically reassembling the packets using the ATM, SAR and CPCS layer protocols. These protocols are also used to detect bit errors and lost or misinserted cells. The logical reassembly process does not necessarily imply that the packet's user information is 
Chapter 6: Conclusions.

reconstructed within the CLSF, nor does it require that the entire packet be received before retransmission can take place. Because of the high bit rate in the BISDN, it is desirable to limit the packet delay as much as possible to prevent data build-up in the system.

The CLSF must re-issue the packets in a way that conforms with the bandwidth specifications on the outgoing virtual circuit, as negotiated with the ATM network. This requires that a logical output queue be maintained for each outgoing connection.

The CLSF design presented in this thesis is intended to conform to the above requirements. It achieves the required throughput by utilising pipelining of operation and special purpose hardware devices to ensure that the average cell proc assing rate is less than the maximum incoming cell rate on the physical link. The cell bodies are removed from their headers to minimise the amount of data copying around the system.

The incoming cells are stripped of their headers by a hardware device and the bodies are placed in $\mathbf{a}$ large buffer. The headers are placed in a FIFO queue, from which they are retrieved by a reassembly device. The first cell header of each packet causes an entry to be placed in a queue corresponding to the desired quality of service, and an area of RAM to be set aside to reassemble the packet's cell headers in. Subsequent cells of the packet are recognised by a CAM search on their headers, causing a pointer to the cell body to be placed in the RAM.

A microprocessor then removes the packet entry from the quality of service queue and uses the destimation address to select an outgoing connection based on a lookup function performed on the cell header. The lookup is based on a tree structure that can examine multiple bits of the destination address at one time. The processor also performs other CLNAP layer functions such as source address validation and charging.

The packet entry is placed in a logical queue corresponding to the outgoing connection, from where another processor removes the packet's cells from the reassembly area, 
adds the new $\mathrm{VCl}$ and places them into a block of memory representing the outgoing cell stream, in such a way that the agreed bandwidth specitications of that connection are met.

The reconstructed cell headers are then shifted into an outgoing cell queue, from where they are taken by another hardware unit which retrieves the cell bodies and forwards the completed cells on the outgoing link.

The operation of this design was tested using computer simulation. It was found that for reasonable assumed values of the processing times. the system operated successfilly under full load at both the standard link rates of $155 \mathrm{Mbps}$ and $622 \mathrm{Mbps}$. The experiment also revealed the limits on the average processing times, beyond which the system fails to operate successfully.

Repeated experiments revealed that the performance of the system is not dependant on the parameters of the system design, but rather on the parameters of the incoming traftic and on the outgoing reserved bandwidths.

An implementation of this design could take advantage of under-utilisation of the processors to reduce hardware costs by using one processor to control more than one functional unit. The effectiveness of this depends on the operating parameters of the actual design.

System capacity can be increased by simply using one of these designs for each input link. The incoming and outgoing virtual circuit connections need to be configured so that packets from any input can get to all the destinations - this requires multiple outgoing connections for each destination.

The requirement that the connectionless server uses the type 4 AAL introduces unnecessary complexity into the system. A simpler, more efficient design could be produced if the direct connectionless service were to make use of the type $5 \mathrm{AAL}$. 
In conclusion, it can be stated that the goals of this thesis have been fulfilled, namely to produce a valid framework for a connectionless server that conforms to the current BISDN standards. The design is feasible, and may be used as a basis for the production of a practical system to be used in future ATM networks. 


\section{Appendix A: Details of ICU Operation.}

This Appendix details the operation of the Input Control Unit hardware device. Refer to Section 3.4 on page 40 for the description of the purpose and the operation of the ICU. Figure 3-6 on page 45 illustrates the layout of the ICU device referred to in the following sections.

\section{A.1 Input Control Tasks.}

The first stage of operation for a particular cell happens before it actually arrives. when a destination address for the new ce!l is read into the address counter from the free space list. The unit then waits until the cell framer indicates that a new cell is being received. This signal causes the cell address to be copied from the counter into the first of the primary registers.

The byte counter is reset, and the octets are counted as they arrive. The first four octets, which comprise the ATM header, are assembled together in the multiplexer. These octets are also input to the HEC checker, which calculates the header checksum. The ATM header is written to the second of the primary registers.

The fifth octet, which is the HEC field, is not written into the multiplexer but it is passed to the HEC checker. If the results of the check indicate an error, or the system/idle cell detector recognises the VCI/VPI pattern as belonging to an idle cell, then the primary registers are reset and the unit goes into an idle state waiting for the next ceil. If the VPI/ VCI pattern indicated a system cell, then the control circuitry is notified via a flag within the system cell detector.

If the ATM header is OK, then the sixth and seventh octets, the SAR sublayer header. are put together in the multiplexer and written into the third primary register. This register 
Appendix A: Details of ICU Operation.

is different from the others in that it is capable of being written to two bytes at a time. The ST value is also noted as it comes in by the control circuitry and the LI checker.

The next 44 octets that arrive, the SAR-PDU payload, are grouped into 32 bit words by the multiplexer and written into both the Cell RAM and the remaining 11 primary registers. The address counter is incremented after each one, while the original address remains in the latch. These octets are also read by the CRC checker.

The sixth word contains the HEL. (Header Extension Length) field in a BOM cell. The value of this field is written into a register in the Transfer Control Unit for later use, regardless of what type of cell this is.

The remaining two octets of the cell, the SAR trailer, are assembled by the multiplexer so as to be read into the second half of the third primary register. The LI field is also noted by the LI indicator. Unless the LI field contains a value of 44 or less, or the LI field value is 63 and the ST indicates an EOM (i.e. an ABORT cell), then the cell is invalid and the system returns to the idle state.

These last two octets include the CRC field and so are also validated by the CRC cher.ker. If a bit error is detected then the unit will again return to the idle state.

If no errors have been detected by this stage then the Input Control unit has completed its task. A signal is sent to the Transfer Control unit to indicate that the header is ready, causing the primary register contents to be transferred to the secondary. Another cell RAM lociation is fetched from the free space list, and the unit begins processing the next incoming cell. 


\section{A.2 Transfer Control Tasks.}

From this point on, the cell is under the jurisdiction of the Transfer Control unit. This unit does no error checking. it simply transfers the header information from the secondary registers into the appropriate queue.

First, the flag in the system/idle cell detector is checked to determine if this is a system cell. If it is, the Secondary registers contain the whole cell. The first word of the cell is the ATM header information. contained in the second of the secondary registers. The second word is the SAR layer protocol information, and the remaining 11 are the cell payload. The Transfer Control thus reads the second through 14th secondary registers and writes them to the System Queue FIFO.

Transferring is done by loading an internal address register with the initial value (in this case two) then loading the Header Counter with the number of words to be transferred (in this case 13). The register pointed to by the address register is copied to the queue, then the address is incremented and the header counter is decremented. Once the header counter reaches zero the transfer stops.

If the cell is not a system cell, then the ST field is examined and used to determine how many words are to be transferred to the Header Queue. If the ST indicates a COM cell, then simply the first three registers are transferred.

If the ST indicates an EOM cell, wien the first 3 words are transferred. The II field is read from a register in the $\mathbf{L I}$ checker, and the secondary register corresponding to that value (using lookup logic if necessary) is written to the queue. If the LI value indicated an ABORT, no words are moved.

If the cell is a BOM or SSM cell, then the first nine words are transferred. The value of the HEL register within the Transfer Control Unit is then copied into the header counter. and that many more words are transferred, up to a maximum of five. 
Appendix A: Details of ICU Operation.

If the cell was SSM, then the LI field is read and used to select a register in the same way as for the EOM cell.

The result of this process is an entry in the Header Queue corresponding to that illustrated in Figure 3-5 on page 43.

The entire transfer takes place in a time frame less than that required to receive the next cell (how much less depends on the technology used). This means that the transfer control must now go into an idle state, waiting for an indication of the next valid cell from the input control unit. When the indication arrives, the entire process is repeated. 


\section{Appendix B: Details of the MRP Operation.}

This Appendix details the sequence of operations followed by the Message Reassembly Processor hardware illustrated in Figure 3-12 on page 57, in order to carry out the tasks described in Section 3.6.2 on page 49. The appendix is divided up into sections corresponding to the different cell types that the MRP must process.

\section{B.1 COM cell operation.}

The procedures followed on reception of a COM cell are the most important to the average processing speed of the MRP, since these cells constitute the vast majority of cells processed. To obtain the highest possible speed, almost all of the COM procedures are performed in special purpose hardware, with the processor being used only to select the appropriate header RAM location for the cell entry. The following list describes the sequence of operations used during the normal processing of a COM cell.

CI Header queue checked for entries.

The hardware control unit monitors the status of the Header Queue. If it has not been disabled by the processor, the control unit will proceed with the following steps as soon as an entry is seen in the queue.

C2 Cell RAM location stored in cell RAM register.

The Cell RAM location is read from the Header Queue and routed by way of the Data Switch to the Cell RAM location register.

C3 ATM header stored in ATM register and multiplexer.

The ATM header word is routed from the Header Queue via the Data Switch to the ATM register. It is also buffered in the upper 32 bit word of the multiplexer.

C4 SAR word stored in SAR register and multiplexer. 
Appendix B: Details of the MRP Operation.

The SAR header queue entry goes from the Header Queue to the SAR register, and also to the lower 32 bit word of the multiplexer. At this point the value of the ST field is tested to determine the cell type.

C5 CAM search performed.

The entire 64 bit multiplexer output, consisting of the two header words, is passed into the CAM and a search is performed using the mask word shown in Figure 3-8 on page 49. This word was pre-loaded into the CAM during system initialisation.

When the search is complete, the CAM indicates the success or failure of the search to find a match. If a match was found, the CAM outputs the entire contents of the connection entry on its data bus.

C6 CAM output written into RAM, SN count and state machines.

The CAM output word is as given in Figure 3-7 on page 48. The memory pointer is stored in the RAM address register to retrieve the rest of the AC table entry. The values of the state variables sar_S and cpes_S are latched into flipflops in the SAR/CPCS state machines. The expected sequence number, rev_SN, is stored in the SN counter.

At this point, the SAR/CPCS state machines have some control over the system operation. If all is normal, the values of sar_S and epes_S should both be REASS, and the following steps continue.

C7 SN checked and incremented.

The $\mathbf{S N}$ counter checks the value of rev_SN against the actual received sequence number stored in the SAR register. A mismatch indicates a cell sequence error. If the values match, the $\mathrm{SN}$ counter increments the value of rev_SN modulo 16.

C8 RAM value retrieved and stored in registers. 
Appendix B: Details of the MRP Operation.

The contents of the RAM location indicated by the address register are retrieved from the RAM as a 64 bit word in the format shown in Figure 3-7 on page 48. The various fields are written directly into the four appropriaie registers, the rev_size register, the BASize register, the BEtag register and the Header RAM location register.

C9U added to rev_size and compared to BA size.

The value of the LI field is read from the SAR register and added to the value of the rcv_size register by the Add unit. The result is written back into the rcv_size register and also compared to the value stored in the BAsize register. If the rev_size is greater than the BASize. an error has occurred.

C10 Header RAM location incremented.

The Header RAM register is incremented to determine the storage location of this cell.

CII Register values written back to CAM.

The updated values of rev_SN, sar_S and cpes_S are written back into the CAM device, along with the current time stamp value.

C12 New register values returned to AC table RAM.

The new values of the rcv_size and Header RAM registers, along with the BEtag and BASize registers, are written back into the AC table RAM at the location specified by the Address register.

\section{C13 Cell RAM location written to Header RAM.}

To do this the hardware unit must gain access to the bus, so it activates the processor's DMA request line as soon as step $\mathrm{Cl0}$ is complete. When access is granted, the contents of the Header RAM register are applied to the address lines, and the appropriate fields from the Cell RAM register and the SAR register are applied to the data bus. The RAM write signal is activated to create a message list entry like those in Figure 3-11 on page 53. 
Appendix B: Details of the MRP Operation.

Once the above steps have been completed at least as far as step C11, the entire process can begin again. A possible timing diagram for this sequence of operations is given in Figure B-1. This diagram illustrates a "best-case" scenario, where the processor is able to answer the DMA request immediately.

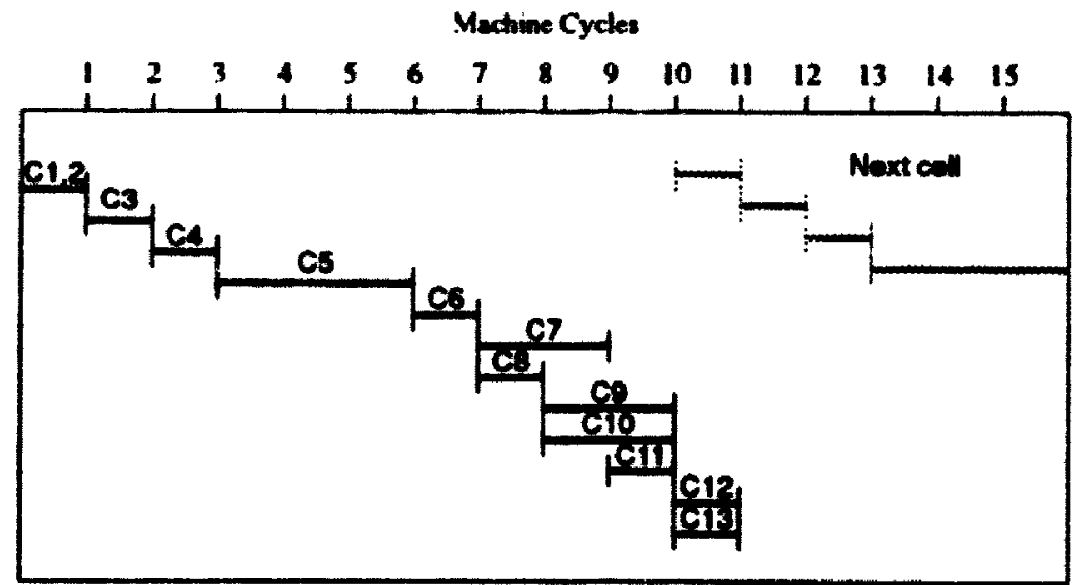

Figure B-1 Timing Diagrem for COM cell processing in MRP

For simplicity, it is assumed that each operation takes about one processor machine cycle, with the exception of the add-and-compares which take two, and the CAM search which takes three. If we assume that the processor has a cycle time of $33 \mathrm{~ns}$ (for a $30 \mathrm{Mhz}$ RISC processor) then the CAM search takes about $100 \mathrm{ns,} \mathrm{which} \mathrm{is} \mathrm{the} \mathrm{current} \mathrm{state} \mathrm{of} \mathrm{the}$ art for such devices. A considerable speedup could be achieved if a fast VLSI technology is used and the system is not synchronised to the processor clock.

\section{B.2 BOM cell operation.}

The sequence of operations followed for a BOM cell is considerably more complex than that required for the COM cell, and takes much more time. Because of the amount of processing needed, the processor is always required to take part in BOM cell handling. The sequence of operations is as follows.

BI-BoSame as for COM cell. 
Appendix B: Details of the MRP Operation.

These are the same as steps $\mathbf{C l}$ to $\mathbf{C 6}$. The values of sar_S and cpes_S should both be IDLE in the absence of errors. At step B4. when the ST field is recognised as BOM the processor is notified of the cell's arrival via an interrupt. The rev_BAsize register is reset to zero as soon as the BOM cell is recognised.

\section{B7 SN field incremented.}

The SN counter does not need to check the value of the SN field for a BOM cell. Instead, it merely increments the value received, ready to store as rev_SN.

B8 CPCS Header word read from quewe into buffers. CPI validated.

The CPCS header word is read from the Header Queue and routed through the data switch to the CPI checker, the BEtag register and the BASize counter. The CPI checker will indicate whether or not the CPI field is valid.

B9 BASize incremented by three.

The BASize counter does this to allow for the length of the PAD field when checking the received message data.

B10 Processor reads five CLNAP header words from Queue.

The processor reads the source and destination addresses and the CLNAP protocol information from the Header Queue. It does this by performing five successive reads ficm a predefined address, causing the address decoder to switch the data from the header queue onto the data bus. The processor stores the destination and source addresses in its internal registers or stack. then examines the fifth word.

BII Processor selects QOS queue and crsates message entry.

After examining the QOS parameter, the processor selects a queue to place the message in. By traversing pointers, it finds the location of the last message entry in the list, and stores the five CLNAP header words in it. If necessary, a new block is retrieved from the free block list. 
Appendix B: Details of the MRP Operation.

\section{B/2 Processor transfers Header Extension.}

The processor examines the HEL field in the fifth CLNAP header word, and uses it to determine how many Header Extension words there are. It then transfers that many words from the Header Queve to the Header RAM, by reading them from the queue as described in step B10 above, then writing them to the RAM locations.

B/3 Processor creates message list.

The processor gets a free block from the free space list, and puts its address in the QOS queue entry. The completed QOS queue entry is as illustrated in Figure 3-10 on page 52.

B14 Processor writes Header RAM location to Header RAM register.

The processor writes the address of the first word in the new message block to the Header RAM register. To do this it simply writes the value of the new RAM location to a pre-determined address. The address decoder recognises the address and instructs the Header RAM register to read the value off the data bus.

B/5 Register values written back to CAM.

Same as for step $\mathrm{Cll}$ in the COM case.

B 10 New register values returned to $A C$ table RAM.

See step $\mathrm{C} 12$ for the COM case.

\section{BI7 Cell RAM location stored in Header RAM.}

See step C13 for the COM case.

Because of the amount of processing done in software, the execution time of the BOM functions will be relatively long. It depends not only on the algorithm, but on the instruction set and speed of execution of the particular processor chosen. An example of the timing diagram for this process is as in Figure B-1. 


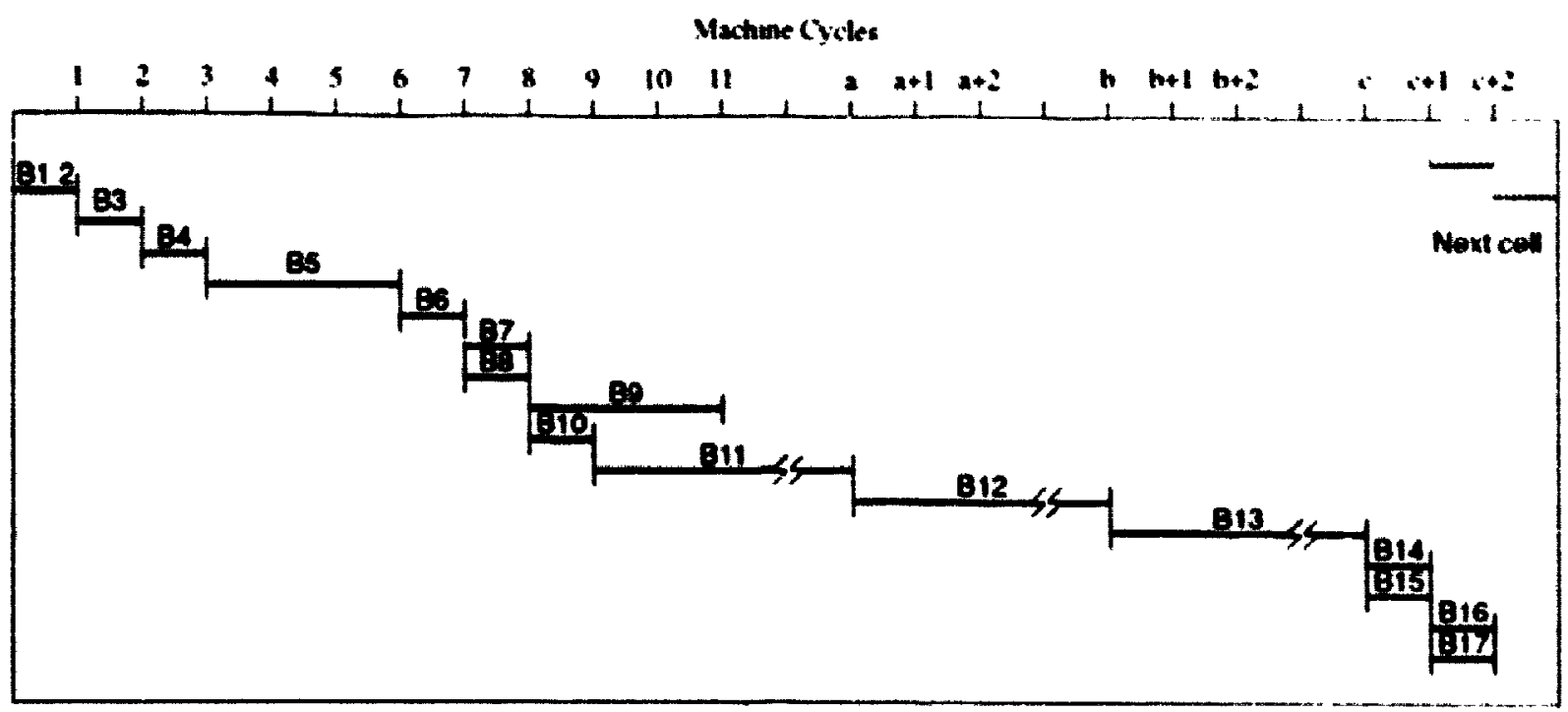

Fare B-2 Timing Diagram for BOM cell processing in MRP

This diagram assumes that the processor is able to answer the interrupt within six clock cycles (from the completion of step B4 when the internupt is generated).

\section{B.3 EOM cell operation.}

Processing EOM cells can be done almost completely in the hardware devices, and so is almost as fast as the COM cell processing. The only major difference between the EOM functions and the COM functions is that the CPCS information is read from the header queve rather than the AC table RAM.

\section{EI-E8Same as for COM cell.}

See steps $\mathrm{Cl}$ to $\mathrm{C8}$ in Section B.I. For this type of cell, however, the LI field is checked as it arrives to ensure that it is not actually an ABORT cell.

E9 Header RAM location compared and incremented

Same as step C10.

EIO CPCS Trailer word read from quewe into buffers, AL and Etag validated.

This is the same process as step B8, with the exception that the AL field is checked to ensure it is all zeros, and the Etag is compared to the BFtag value 
Appendix B: Details of the MRP Operation.

rather than overwriting it. A mismatch of either of these two indicates an error.

The length field is written into the BAsize register.

EII W added to rev_size and compared to Length field.

This is similar to step C9. If the result of the addition is not equal to the received Length field then an error has occirred.

E/2 Register values written back to CAM.

Same as step CII. The values of sar_S and cpcs_S will both be IDLE.

EI3 Cell RAM location written to Header RAM.

Same as step C13.

The timing diagram for the EOM cell operations is illustrated in Figure B-1. This also assumes that the processor answers the DMA request immediately.

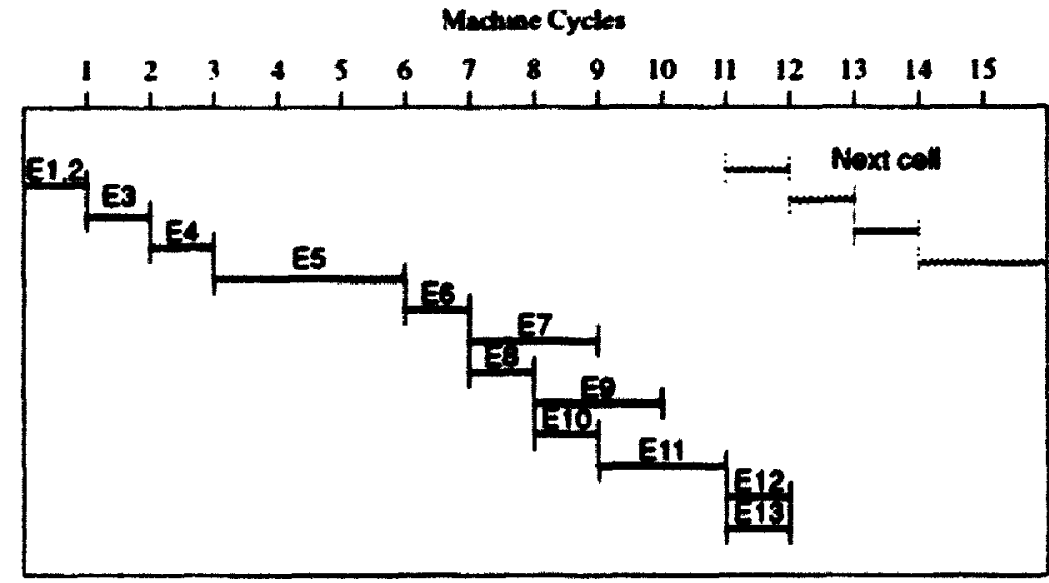

Figare B-3 Timing Diagrem for EOM cell processing in MRP

\section{B.4 SSM cell operation.}

A single segment message (SSM) cell is effectively a combination of a BOM cell and an EOM cell. To deal with these cells, the system must draw from the procedures from hoth types of cells. Because a new message is being created, the processor is involved and so the SSM cell takes a relatively long time to process. 
Appendix B: Details of the MRP Operation.

To handle a SSM cell, the MRP follows a subset of the BOM procedures, then a subset of the EOM procedures. Steps S1 to S14 are the same as steps B1 to B14, while steps Sis to S18 are the same as steps E10 to E13. Steps S1 to S14 read the ATM. SAR and CPCS headers into hardware, create a new message entry in the Header RAM, and copy the CLNAP information across into the RAM. Steps SI5 to S18 read the CPCS trailer into hardware and verify it, then write the cell entry into the message list in header RAM. This re-use of the same procedures simplifies the hardware system design.

\section{B.5 ABORT cell operation.}

An ABORT cell header queue entry has a ST value of EOM. but the value of the I.I field is unique to this type of cell. The action taken on receipt of an ABORT cell involves discarding the current message and setting the SAR and CPCS processes back to /I)/F.

The procedures are as follows.

Al-A6Same as for COM cell.

See steps $\mathrm{Cl}$ to $\mathrm{C} 6$ in Section B.1. At step A4 the Abort detector recognises the LI field as belonging to an ABORT cell. The processor is notified via an interrupt that an ABORT cell has been received.

A7 RAM value retrieved and stored in registers.

Same as step C8.

A8 Processor fetches Header RAM location.

The processor fetches the Header RAM location from the Header RAM register by reading from a pre-defined address. The Address decoder recognises this address and signals the Header RAM register to output its contents onto the data bus.

A9 Processor discards message. 
Appendix B: Details of the MRP Operation.

The processor traces back through the message list until it finds the first untransmitted cell. As it goes, the cell RAM locations of all the cells are written to the Free Space List via the data switch.

The number of copies field is checked to see if the Routing Processor has processed this message yet. If it hasn't, the block is emptied and returned to the free block list. The QOS queue entry is found from the pointers and nullified by setting all the words to zero.

If the RP has processed the message, then the first cell in the message i:s checked to see if the Bandwidth Control Processor has begun transmitting the message. If the cell is a $50 \mathrm{OM}$ cell, the block is emptied and the connection queve entry nullified. Otherwise, the untransmitted cells in the block are emptied. The LI and ST fields of the first untransmitted cell entry are changed to the ABORT values, so that the BCP will issue an ABORT cell, and the pointer: are changed so that there is now only one cell in the queue. The Abort bit in the message block is set.

During this procedure, the other interrupts remain enabled so the processor can continue to process incoming BOM cells.

Alo Process state written back to CAM.

As for step C11. The SAR and CPCS processes are both in an IDLE state after this cell has been received.

The timing diagram for the ABORT cell is shown in Figure B-1. Note that the system need not wait for the processor to finish with the cell before it can begin on the new cell processing. It does, however, need to wait for the processor to answer the interrupt. 


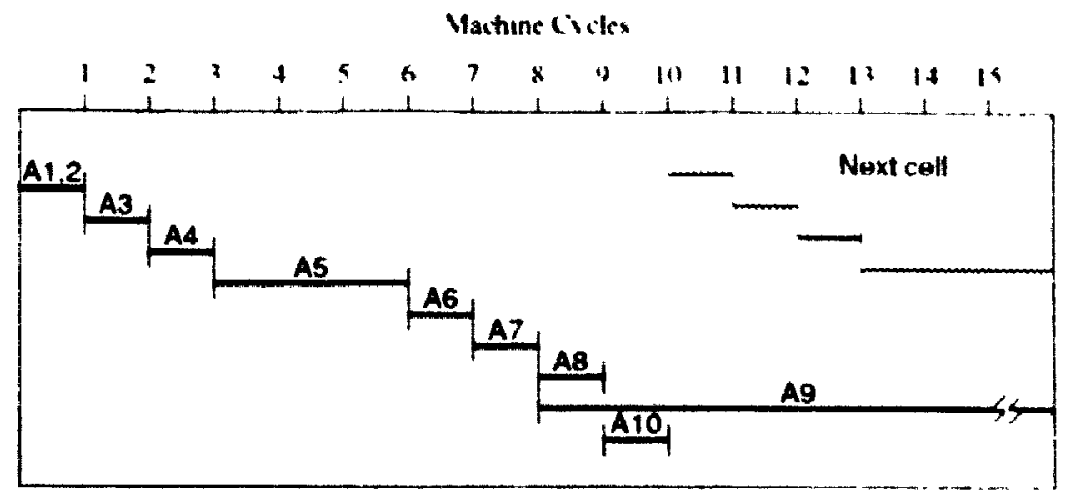

Figure B-4 Timing Diagram for ABORT cell processing in MRP

\section{B.6 Error Handling procedures.}

On reception of an errored cell, the processor is called upon to perform error handling procedures. This normally involves discarding the current message, in the same way as for the ABORT case.

The first error that can be detected by the MRP is an error in the connection identitict fields, i.e. an illegal connection. This will be identitied by a CAM search that fails to find a match. If this occurs the MRP will simply discard the cell by writing the cell RAM Ination from the cell RAM register to the Free Space List.

A cell sequence error will be detected by the SN tield failing to match that stored in the SN counter. Such an event requires that the current message be discarded. The cell RAM location will be put on the Free Space list as above, and the processor notitied of the error via an interrupt. The MRP will abort the message using the same sequence of events as in the ABORT cell case of Section B.5. The timing diagram is the same for that of Figure B1, with the cell RAM location going to the FSL cccurring in parallel to step A7. The SAK and CPCS processes are both returned to the IDLE state.

An error at the CPCS level such as an illegal CPI value or a received size greater than the rev_BASize value, will cause the message to be aborted in the same way. The only difference is that the CPCS reassembly process returns to the $A B O K T$ instead of the $/ / H F$ state if the cell was BOM or COM. 
Appendix B: Details of the MRP Operation.

If the SAR and CPCS processes are in the IDLE or ABORT states when a COM or EOM cell arrives, then the current message is being aborted. As soon as the state of the processes is known, the cell is immediately discarded as described above.

\section{B.7 Setup and Maintenance Procedures.}

Before the system can function correctly, the Active Connection table needs to be set up correctly. It is also important that the contents of the table can be altered dynamically, as new connections are established and removed. It is the System Manager that is responsible for these tasks, which it achieves through use of the system queue.

A new connection will be added to the AC table either when the system is being initialised, or when an external device signals the System Manager to do so during network operation. To place a new connection entry in the table, the System Manager simply places a control message in the system queue. The message entry consists of a control word followed by two data words. The data words comprise the CAM part of the AC table entry.

The action of placing a message in the queue generates an interrupt in the processor, which then reads the control word from the queue. Recognising the word as being an instruction to add an entry, it signals the hardware by writing to a predefined address.

Once it compietes its current cell the hardware then reads the two data words from the system queue. The entry passes through the data switch and the multiplexer and is written into the CAM device. The corresponding RAM location is not upuiaicd, as it will only contain information that will be generated when the first message arrives.

Deleting entries from the table occurs in much the same way. The System Manager puts a three word entry in the queue, generating an interrupt. The data words in this case are used to locate the entry in the CAM in order that it can be deleted, so the CAM mask is used and a search is performed. Control signals tell the CAM to delete the matching entry. 
Similar procedures can be used to instruct the MRP to reset itself. clear the CAM. generate a status report, etc.

Periodically, the processor will step through the CAM contents to check the time stamps on the connections. To do this, it must have maintained its own record of all the connection identifiers stored in the CAM. The processor first writes the expired time stamp value into the time stamp generator. It then steps through the list. searching for each of the connection identifiers in the CAM. The time stamp generator then compares the time stamp value from the CAM to the value stored in itself. If they are the same, the hardware system aborts the current message on that connection in the same way as for the ABORT cell. Once some or all of the connections have been checked, the processur increments the current time stamp value and returns it to the time stamp generator, allowing the system to continue with normal operation. 


\section{Appendix C: Details of the OCU operation.}

\section{C.1 Normal cell procedures.}

At the start of each cell, the OCU control tests the state of the System Queue to determine if the System Manager has inserted any cells to be transmitted. If the queue is empty, the OCU then checks to see if there are any cell headers in the Header Queue. If there is, the $\mathrm{OCU}$ begins its cell transmission procedures.

First, the cell RAM location is read from the header queue and latched into the address counter. The second word in the header queue contains the ATM cell header information, and it is read from the queue and latched into the multiplexer buffer. The multiplexer begins sending out the four ATM header octets one at a time, both to the framer circuit and to the HEC generator circuit. Once the four octets have been transmitted, the HEC generator issues the fifth ATM header octet containing the HEC field.

The last word in the header queue entry, containing the SAR protocol infermation. is then read from the header queue and latched into the multiplexer. The LI field is also latched into the LI latch, and tested to cietermine if the entry represents an ABORT cell. The two SAR octets are read from the multiplexer and are sent to both the cell framer and the CRC generator circuit.

The address counter then sends the cell RAM location to the cell RAM, reading out the first word of the cell payload into the multiplexer. The multiplexer sends the data to both the framer and the CRC generator, one octet at a time. The address counter increments the address and reads out the successive data words from the RAM, until all 11 data words have been transmitted. 
Appendix C: Details of the OCU ipperation.

Finally, the LI field is read from the LI latch and passes straight through the multiplexer to the CRC generator circuit. The CRC generator outputs the two SAR-PDU trailer octets, containing the LI and CRC fields, to the framer circuit.

If the Discard bit is set, the address generator then copies the original cell RAM lowation to the Free Space List. The entire procedure begins again immediately for the next cell.

\section{C.2 System/Idle/Abort cell procedures.}

The procedure followed for a system cell is very similar to that used for normal cells. except that all the cell information comes from the System Queue. The system queue entry format contains a header similar to that shown in Figure 3-18 on page 69 but without the cell memory location, followed immediately by 11 data words. The $\mathrm{OCU}$ reads the header from the queue and transmits it as for no:mal cells, then reads the 11 data words from the cell and transmits them. The SAR trailer is appended in the same way as above, and the unit begins its routine again. No address is written to the address counter or the Free Space List in this case.

If both queues are empty at the beginning of a cycle or the header quete contains an idle cell entry, the $\mathrm{OCU}$ transmits an idle cell. It does this by intemally generating its own ATM header and cell data, using the Idle/Abort cell generator. This generator initially produces a valid idle ATM cell header word, which is multiplexed and transmitted in the same way as for the other types of cells. The unit then generates 12 data words which form the idle cell payload, and the st: stem transmits them one at a time. The data words consist of a special pattern used in idle cells, such as all zeros. The CRC generator does not operate on idle cells. Once all 12 words have been transmitted a new cycle begins.

During the transmission of an ABORT cell, the $\mathrm{LI}$ will indicate the cell type during the transmission of the SAR header. This means that the cell RAM location is invalid, and so 
the address counter is not used in this case. Instead, the Idle/Abort cell generator provides the cell payload, which consists of 11 words of all zeros. Once these words have been transmitted, the $\mathrm{LI}$ is sent and the CRC field generated in the same way as before. 


\section{Appendix D: The Simulation Model.}

The CLSF system was modeled using a discrete event simulation program written in C. The approach taken was to create an event-driven simulation, where various events within the system are scheduled to occur at different times.

The operation of the simulation program is as follows. The program contains a number of subroutines, representing the five major components of the basic system: the external sources, the Incoming/Outgoing cell units, the Message Reassembly Processor. the Routing Processor, and the Bandwidth Control Processor. Within the main loop of the program. a "future event list" is maintained, which schedules the next occurrence of an event in each of the system components. During a simulation run, the system clock is advanced to the time of occurrence of the first event on the list and the subroutine corresponding to that event is called. The execution of the subroutine will usually cause the next occurrence of that or another event to be scheduled and added to the list.

The program models the movement of user messages through the CLSF system by storing the messages in memory and passing pointers to them among the subroutines. The input sources generate packets, which represent CLNAP idyer messages. Each packet is: stored as a data structure within the program, containing its source and destination addresses, quality of service, statistics gathering information, and a pointer to an area of memory containing the cells which make up the packet. Each cell contains an identification and a sequence number, as well as statistics gathering information.

At the input to the CLSF, cells are generated at the input link and examined by the ICU for errors. Pointers to the cells are placed in the Incoming Header queue. The MRP subroutine then examines the cells at the head of the queue, checking for sequence errors. For BOM cells, a pointer to the corresponding packet entry is placed in the appropriate Quality of Service queue. The Routing Processor subroutine removes the pointer from the QOS 
Appendix D: The Simulation Model.

queue and places it in the desired Outgoing Connection queue, or multiple queues if the packet is to be multicast. The Bandwidth Control Processor subroutine scans the Outgoing Connection queues, and places pointers to the packet's cells in the Outgoing Header queue. from where the $\mathrm{OCU}$ routine removes them from the system.

By manipulating pointers in this way the simulation program is able to model the movement of large amounts of data, without consuming large amounts of memory. As a representation of each packet is created, memory is allocated to store its cells' information, and as the packet leaves the system that memory is freed again for re-use by other programs. Only pointers to the memory areas are copied around the system, thus also improving the speed of the program.

The program is set up so as to be able to repeatedly execute a particular simulation run using different random number streams, allowing a 95\% confidence interval to be determined for any given output result. In addition to running the simulation multiple times, the series of runs can be repeated with a different value of a particular system parameter. In this way the variation of the simulation results with the system parameter can be plotted.

The $\mathrm{C}$ source code for this simulation program is approximately 1700 lines in length. The resulting executable takes about 30 seconds to simulate 1 second of system time on a Sun SPARCstation 2. when the input link is fully loaded at 155 Mbps. Modelling a 622 Mbps system takes about 2 minutes 5 seconds per second of simulation time.

\section{D.1 Input Trafific Model.}

The input traffic is generated by a subroutine that models the input sources of the system. The routine generates bursts of packets that correspond to user messages, according to the parameters described below. The routine is called whenever a burst is scheduled to begin on any one connection. 
Appendix D: The Simulation Model.

The input to the CLSF takes the form of a single physical link. operating at either $155 \mathrm{Mbps}$ or $622 \mathrm{Mbps}$. To this link is attached a number of sources, each allocated it specific portion of the link bandwidth. A source issues cells at an average rate corresponding to its allocated bandwidth. Cells from each of the sources are multiplexed together at random. From the point of view of the CLSF this setup is equivalent to the real situation. where the sources are attached via an ATM network but share a common input link using statistical multiplexing.

To model the traffic generated by each source, the source is considered to have two staves, burst and idle. A burst corresponds to a period during which the source is transmitting a message to one or more of the other sources via the CLSF. Between bursts the source is idle and does not contribute any input to the system. The length of a burst period is simply the time taken to transmit all the cells of the message. The length of the idle period is chosen so as to maintain the bandwidth allocation for the source.

The number of cells issued during a burst depends solely on the length distribution of the messages which could be expected to flow in a real system. Unfortunately. much of the traffic that will utilise the direct BISDN connectionless serva timedia or other high-bandwidth applications that are not in common use today. As a result, it is impossible to predict what the real message parameters will be. Instead, for the purposes of this model the message length is considered to be exponentially distributed. with a mean that can be varied to determine its effect on the system. As in the real situation, lengths are rounded up to the nearest cell.

The message length is generated according to the following formula:

$$
t_{n}=\lceil-i \operatorname{kg} R\rceil
$$

where $l_{n}$ is the length of message $n$ (in cells), $l$ is the mean burst length (in cells), and $R$ is a uniform $(0,1)$ random variable. Note that the length is rounded up to the nearest cell. 
The idle time between bursts on a particular connection is chosen so that the actual mean bandwidth on the connection always equals its allocated mean bandwidth. To achieve this, the time at which a burst starts and its length are recorded. When the burst is complete, the mean bandwidth allocation for that connection is used to calculate how much time the burst should have taken (had it been transmitted at exactly the mean rate), and the next burst is scheduled to start at the end of that period. The calculation is as follows:

$$
t_{n+1}=t_{n}+\frac{t_{n} T_{c}}{b}
$$

where $t_{n}$ is the idle time after message $n . T_{c}$ is the cell transmission time, and $b$ is the propertion of the bandwidth allocated to this connection.

This means that the input does not directly model a true system, in the sense that burst arrival times are not truly random. This is necessary in order to be able to control the amount of input traffic seen by the system. The model of a particular source could instead be thought of as representing a LAN gateway that always has a non-empty queue of packets waiting to be sent. so that it is continually issuing packets at exactly its mean bandwidth allowance.

To model the switching aspects of the CLSF. each burst is assigned a destination to which the message is to be sent. The destination is chosen at random from among the list of sources. To avoid congestion of messages within the system, the probability of a particular destination being chosen is equal to the fraction of the total link bandwidth assigned to it. This ensures that the mean bandwidth allocation for the destination is not exceeded on the average.

Some of the bursts will be assigned multiple destinations, representing a multicast message. The probability of a message being multicast is one of the system definitions that may be varied. 
Appendix D: The Simulation Model.

The principle behind this model is not to ensure that the bandwidth allociation acicurately refiects the actual traffic, as would be the case in a real ATM network. That would involve getting into the complex problem of traffic allocation algorithms. which is heyond the scope of this thesis. Instead. the traftic is chosen so as to accurately reflect the bandwidth allocation. The effect on the system as a whole is the same.

\section{D.2 Input/Output Model.}

The Input/Output of the CLSF system model represents both the incoming and outgoing physical links and the Incoming and Outgoing Cell Units. It is this subroutine that performs the statistical multiplexing on the input link, the error detecting in the ICU, the placing and removing of cells from the incoming and outgoing cell queues respectively. and the maintaining of cell RAM statistics. Events in all these places occur periodically and simultaneously, in synchronisation with the incoming and outgoing celi streams.

\section{D.2.1 Statistical Multiplexing.}

Once every cell transmission time, the model randomly chooses a connection from among those that are in the middle of a burst, and generates a cell from that conuection. If necessary, a new packet is created with the characteristics of the current burst. Note that according to the CCITT definition of the CLNAP protocol ${ }^{|5| \mid}$, the maximum packet size is 9188 octets or 210 cells. Hence, any burst longer that 210 cells will be broken up into a series of packets of $\mathbf{2 1 0}$ cells or less.

The random choice of connection models the statistical multiplexing effect of the ATM protocol. The probability of a connection being chusen is the inverse of the number of active connections at that particular point in time. Since not all connections are likely to be active at once, the short-term bandwidth on a given active connection is normally greater than the mean bandwidth, up to a maximum of the link bit rate. 
In the true situation the maximum cell rate on a connection is likely to be capped at a peak rate which is less than the link capacity. Also, some of the link capacity is taken up by physical layer overhead, so that the occasional cell will always be empty. The second problem is mainly taken care of by specifying a link capacity that excludes the physical overhead, but the first represents a small departure from the true situation. It will be seen from the results, though, that this has little effect on the system as a whole.

\section{D.2.2 ICU model.}

The main function of the ICU in the real system is the separation of the cell headers from their bodies. This is not required in the model as there is no actual data in the cells. However, the ICU also detects errored cells, which can have an effect on the CLSF simulation. The input model takes into account this function of the ICU.

The time taken by the ICU to process the cell is always exactly one transmission time. This factor is taken into account when calculating packet and cell delays in the model.

The probability that a cell contains a bit error or is lost in the system can be specified as a simulation parameter. For each cell that is generated, the occurrence of an error causes it to be discarded immediately. Otherwise, a pointer to the cell is placed in the incoming header queue. The Cell RAM statistics are updated at this point.

In order to limit the memory usage of the simulation program, a limit was put on the maximum size of the input queue. If the input queue is full, then the incoming cell is discarded. The same condition applies to the outgoing header queue. Under most situations these values will not be exceeded, and so the presence of limit will not affect the simulation results. A queue size limit will also exist in the real system, but may well be larger than that used in the simulation.

\section{D.2.3 Output Model.}

There is no need for any multiplexing to be done at the output, as this is taken care of by the Bandwidth Control Processor. As a result the output model is very simple, involv- 
Appendix D: The Simulation Misdel.

136

ing only the removal of cells from the output queue (if there are any). The transmitted cells are then discarded from the system.

\section{D.3 MRP Model.}

The MRP subroutine carries out the same sorts of functions as the Message Reassembly Processor in the original design. Like the true MRP. it keeps track of the expected sequence number and the connection status of each incoming connection. Inlike the rea system though, the model does not need to perform a CAM search for each cell that comes in as the cells do not have VCI numbers.

The MRP subroutine is called whenever a new cell is placed in an empty incoming header queue, or when it is scheduled to tinish processing its current cell. The mixtel removes the cell from the head of the queue and checks the connection status of the cell's source. It then calculates how long the MRP hardware would take to process that cell, and schedules the next calling of the subroutine according to the processing times listed in Table 4-3 on page 79.

The next time the routine is called, the header RAM is updated. For a BOM cell. this involves placing a pointer to the packet entry into the appropriate QOS queue. The MRP then removes the next eritry from the queue if there is one.

By introducing a time delay between the removal of the cell from the input queue and the placing of the cell in the Header RAM, the processing time of the real MRP hardware is simulated. When an operation such as the BOM processing which is partly carried out in software is performed, the amount of time that the MRP processor spends on the cell is recorded. This allows statistics relating to the MRP processor usage to be obtained.

Should an error be found, then the status for that connection is set to abort. When the status is abort, all subsequent cells from that packet are dropped until the EOM cell is seen. Also, the MRP routine searches through the QOS and connection queues until it 
Appendix D: The Simulation Model.

fincls a pointer to the packet, and changes the pointer to indicate that the packet has been dropped. This is equivalent to the action taken in the real system. where the entry is cancelled tut not removed from the queue by the MRP (since it may be in the middle of a b!ock).

The times taken by the MRP processor to remove cel! and packet entries from the Header RAM are also added to the processor usage statistics, but they do not affect the scheduling of the MRP routine as they are carried out in the background in software in the real system (see Apperidix B.5). Table 4-3 Also lists the default software execution times for these tasks that were used in the simulations.

\section{D.4 RP Model.}

The model of the Routing Processor in this system is very simple. as the main job of the RP is simply to remove entries from the QOS queues and place them in the appropriate connection queues. The RP scans the QOS queues in order from highest to lowest priority. only removing the packets from a given queue when all higher priority queues are exhausted.

When the first message is placed in one of the QOS queues, the RP subroutine is called after a short scan time (representing the t.me taken for the RP to notice that the QOS queue is no longer empty). The RP subroutine removes the packet pointer from the head of the queue, and reschedules itself to be called again one processing time later. In this model. the execution time for the Routing Processor to process a packet - assumed to be the same for all packets and is given in Table 4-4 on page 80.

Once the RP routine is called again, it places a pointer to the packet in the appropriate outgoing connection queue. If the packet is multicast, then copies of the pointer are put in each of the packet's destination queues. 
Appendix D: The Simulation Mindel.

It is assumed for the sake of simplicity that there are no incorrectly addressed or forged messages, so the tasks of source and destination address validation are not required. As the probability of occurrence of these errored palkets is likely to the extremely low in a real system anyway. this assumption does not have an effect on the model.

\section{D.5 BCP Model.}

The BCP model is subdivided into two parts, one representing the BCP processor and one representing the DMA hardware. The processor model scans through the connection queues at fixed intervals, and checks for non-empty pickets. When it tinds one, it determines how many cells to transmit on that connection and copies them to a cell stream reconstruction area. At the end of a scan it schedules an occurrence of the hardware model, which places a cell in the queue and reschedules itself until all the cells have heen transmitted.

\section{D.5.1 Processor Model.}

The model of the BCP processor is responsible for carrying out the bandwidth control algorithm described in Section 3.8.1.1 on page 65. The outgoing cell stream is iplit into batches of cells, the lengths of which are determined by the BCP scan size parameter. During each scan, the BCP processor routine visits one connection queue at a time. It places cell pointers from that queue in the CSC area so that the mean and peak bandwidth allocations of the connection are not exceeded.

The BCP routine scans the connection queues by visiting a different queue each time it is called by the scheduling routine. At each visit, it calculates the maximum number of cells that can be transmitted without exceeding the mean bandwidth allocation.

Once the maximum number of cells to transmit on the connection are known, the $B(\cdot P$ routine begins copying cells to the CSC area. Once the entire packet has been copicit then 
Appendix D: The Simulation Model.

the BCP removes it from the queue and begins on the next packet. It continues copying cells until either the maximum is reached, the end of the CSC area is reached, the queue is empty or all received cells in an incomplete head of queve packet have been copied.

The cell pointers are spaced within the area so as to adhere to the peak bandwidth specification. As each cell is copied, the BCP processor usage statistics and the time of the next connection start are increased by the cell copy time parameter value. This ensures that the time taken to copy the cells is modeled.

In the case of a multicast packet, the BCP model also determines how many cells are being transmitted for the last time by calculating the lowest difference between the number of cells transmitted so far on this connection and the number transmitted to the packet's other (ut.inations. If the value is positive then this many of the connection's cells will be flagged as they are placed in the area.

The routine then reschedules itself to begin processing the next connection. When all the connections have been examined, the processor routine schedules an occurrence of the BCP hardware routine (if it is not still busy), and reschedules itself to be called again at the start of the next scan cycle. If the BCP hardware is still busy, the processor will wait until it is free.

The above procedures mimic the operation of the real system, in which the BCP processor constructs the output stream one connection at a time, then activates the hardware to copy the stream to the output queue while the bandwidth calculations for the next connection occur in the background.

\section{D.5.2 BCP hardware model.}

The operation of the BCP DMA hardware is very simple, and so is that of its model. When called, the routine places a pointer to its current cell in the output header queue. If the cell is to be discarded. a tield in the output queue is set. It then removes the next cell 
from the header RAM. and reschedules itself to occur one shift time later. The shift time is given in Table 4-5 on page 81.

Once all the cells have been shifted, the model removes itself from the scheduling list. It will be called again once the next scan processing is complete. 


\section{References.}

11] B. Amin-Salehi and D. R. Spears, "Support of transport services in BISDN," in GLOBECOM '89, pp. 1467-1472. Nov. 1989.

121 G. Bucci, A. Del Bimbo, and S. Santini, "Fast filtering in bridging Fthernet and FDDI networks: a CAM approach," Ninth Annual Intemational Phoenix Conference on Computers and Communications, (Scottsdale, AZ), pp866-867, Mar. 1990.

13) W. R. Byrne, G. W. R. Luderer, G. Clapp, and H. J. Kafka, "Evolution of metropolitan area networks to Broadband ISDN," IEEE Communications Magazine, pp. 69-82. Jan. 1991.

14] V. Catania, A. Puliafito, and L. Vita, “A DQDB MAN switching system supporting a high speed connectionless data service.," in ICC '91, (Denver), pp. 1068-1074, IEEE. June 1991.

15] CCITT Draft Recommendation F.812, "Broadband connectionless data bearer service."

[6] CCITT Recommendation E.164, "Numbering plan for the ISDN era."

17] CCITT Study Group XVIII, “B-ISDN functional characteristics," Draft Recommendation I.211. CCITT, Geneva, May 1990.

[8] CCITT Study Group XVIII, "B-ISDN functional architecture," Draft Recommendation 1.327. CCITT, Geneva. May 1990.

|9] CCITT Study Group XVIII. "B-ISDN ATM adaption layer (AAL) functional description." Draft Recommendation I.362. CCITT, Geneva. May 1990. 
[10] CCITT Study Group XVIII. "B-ISDN ATM adaption layer (AAL.) speciticaltion," Draft Recommendation 1.363. CCITT, Geneva. May 199(1.

[11] Chairman SWP 8/5, "Report of SWP XVIII 8/5 AAL." tech. rep., CCITT, Mtatsuyama. Dec. 1990.

[12] L. Chisvin and R. J. Duckworth. "Content-addressable and associative memory: alternatives to the ubiquitous RAM," IEEE Computer magazine, pp. 51-64, July (4)4".

[13] G. A. Clapp, "Routing of interconnected networks and extended LANs." in Broadband (FOC/LAN) '89, (San Francisco), pp. 183-187, Oc1. 1989.

[14] G. H. Clapp, "LAN interconnection across SMDS," IEEE Network, pp. 25-32. Sept. 1091.

[15] J-P. Coudreuse, "Network Evolution towards BISDN," IEEE LTS, pp. 66-71). Aug. 1991.

[16] P. Crocetti, G. Gallasi, and M. Gerla, "Bandwidth advertising for MAN/ATM connectionless internetting.," in INFOCOM '91, (Bal Harbour, FL), pp. 1145-1151. IEEE. Apr. 1991.

[17] B. S. Davie, "A host-network interface architecture for ATM," in SICiCOMM '91, (Zurich), pp. 307-315, Sep. 1991.

[18] F. Dix, M. Kelly, and R. Klessig, "Metropolitan area networks and switched multi-megabit data service," in Broadband (FOC/LAN) '89, (San Francisco), pp. 177-182. Oct. 1989.

[19] G. Fioretto, T. Demaria, R. Vaglio, A. Forcina, and T. Moro, "Connectionless service handling within B-ISDN," in GLOBECOM '91, pp. 217-222, 1991. 
[20) W. Fischer, E.-H. Goeldner, and N. Huang, "The evolution from LAN/MAN to Broadband ISDN.," in ICC '91, (Denver), pp. 1251-1257. IEEE, June 1991.

|21| E. H. Goeldner and M. N. Hugbr. "Multiple access for BISDN," IEEE LTS, pp. 37-43, Aug. 1991.

|22] E. H. Goeldner and M. N. Huber, "Network evolution towards BISDN," IEEE LTS, pp. 66-70, Aug. 1991.

|23| G. J. Heijenk and I. G. Niemegeers, "Variable bandwidth connections for a connectionless service on ATM - performance modelling and evaluation.," in IFIP Workshop on Broadband Communications, (Portugal), pp. 330-341, Jan. 1992.

[24] C. F. Hemrick, R. W. Klessig, and J. M. McRoberts, "Switched multimegabit data service and early availability via MAN technology." IEEE Communications Magazine, vol. 26. pp. 9-14, Apr. 1988.

$125 \mid$ S. lisaku and M. Ishikura, "ATM network architecture for supporting the connectionless service," in INFOCOM '90, pp. 796-802, IEEE, 1990.

[26] G. Kaplan (editor), "Special guide to data communications: High-speed networks and interconnection," IEEE Spectrum, pp. 22-44, Aug. 1991.

[27] KDD Japan, "Proposed layering of an AAL for connectionless data services," Delayed Contribution D.581/XVIII, CCITT, Geneva, Jan. 1990.

[28] KDD Japan. "Proposed cell routing functions of an AAL for connectionless data services," Delayed Contribution D.582/XVIII, CCITT, Geneva, Jan. 1990.

|29| T. J. King, T. J. A. Barker, A. C. Micallef, and K. W. Mackay, "Asynchronous transfer mode (ATM) - practical management issues.," British Telecom Technology Journal, vol. 9. pp. 57-70, Apr. 1991. 
[30] T. J. King, R. Rowley, G. M. Whalley, J. Clementson. and J. Chauhan. "The asynchronous transfer mode technology platform - initial laboratory trials." British Telecom Technology Journal, vol. 9, pp. 71-83. Apr. 1991.

[31] W. S. Lai, "Broadband ISDN bandwidth efticiency in the support of ISO connectionless network service.." in ICC '91, (Denver), pp. (1983-(1987, IFEE. June 1991.

[32] T. van Landegem and R. Peschi, "Managing a connectionless virtual overlay network on top of an ATM network.," in ICC '91, (Denver), pp. (0988-1)992. IEEE. June 1991.

[33] A. Lespagnol and T. Houdoin, "A high speed transport protocol on an ATM network," in EFOC/LAN '89, 1989.

[34] B.C. Lindberg, "Switched broadband network interfaces," Telecommunications magazine (North American edition), pp. 47-58, Oct. 1991.

[35] M. Listanti and R. Winkler, "Addressing and control issues for movability features in the B-ISDN connectionless service," in IFIP Workshop on Broadband Comnunications, (Portugal). pp. 45-56, Jan. 1992.

[э6] M. Lobjinski, G. Horn, and M. Horn, "Realization of broadband data services in ATM ne:works," in IZS '90, (Zurich), pp. 73-86, IEEE, Mar. 1990).

[37] B. Materna, B. J. N. Vaughan, and C. W. Britney, "Evolution from I.AN and MAN networks towards the integrated ATM network," in GLOBECOM '89. pp. 14551461, Nov. 1989.

[38] Microtel Pacific Research (MPR) Canada, "Support of connectionless data service using virtual ring topology," Study Group XVIII Contribution D.620, CCITT. Dec. 1989. 
References.

|39| L. Mongiovi, M. Farrell, and V. Trecordi, “A proposal for interconnecting FDDI networks through B-ISDN," in INFOCOM '91, (Bal Harbour, FL), pp. 1160-1167, IEEE, Apr. 1991.

[41] D. S. Omundsen, "The role of the connectionless service in Broadband ISDN: A constructive survey of the literature," Carleton University Systems Engineering report SCE-92-(12, Nov. 1991.

[4I| B. Pauwels, "Terminal-to-network communication in an ATM-based ISDN," International Journal of Analog and Digital Cabled Systems, vol. 2, no. 4, pp. 49-56. 1989.

142] T. B. Pei and C. Zukowski, "Putting rouing tables in silicon," IEEE Network Magzine, pp. 42-50, Jan. 1992.

|43] M. de Prycker, "Impact of data communication on ATM," in ICC '89, (Boston), pp. 705-711. IEEE. June 1989.

[44] M. de Prycker. Asynchronous Transfer Mode - Solution for Broadband ISDN. Series on Computer Communications and Networking. Ellis Horwood, 1991.

145] M. de Prycker, R. Peschi, and T. van Landegem, "B-ISDN and the OSI protocol reference model," in Third IFIP Conference on High Speed Networking. (Berlin), pp. 39. 57. IFIP. Mar. 1991.

[46] Royal PTT Nederland NV. "Need for a simple and efficient adaption layer (SEAL)," Study Group XVIII Contribution D.1966, CCITT, Dec. 1991.

|47| J. Shantz, "SMDS: an altemative to private networks?," Telecommunications Magazine (North American edition), May 1989.

[48] T1S1. "Convergence sublayer protocol: PDU structure and common header/ trailer format," Study Group XVIII Contribution AAL-10, CCITT, June 1991. 
Refenences.

[49] C. B. S. Traw, J. M. Smith. “A High-performance host interface for ATM networks," in SIGCOMM '91. (Zurich). pp. 317-325, Sep. 1991.

[50] R. Vickers and M. Wernik. "The role of SDH and ATM in broadband acceess networks," in GLOBECOM '91. pp. 212-216. 1991.

[51] Working Group TIS 1.5. "Draft CCITT BISDN Recommendations - Part 1". January 1992.

[52] Working Group TIS1.5, "Draft Cr.ITT BISDN Recommendations - Part 2". January 1992.

[53] Working Group TIS1.5. "S - TT SG XVIII Working Party VIII Meeting Report". January 1992.

[54] Y. Ujihashi, T. Shikama, K. Watanabe, and S. Aoyama, "An architecture for connectioniess data service in B-ISDN," in GLOBECOM '91), pp. 1739-1743. 19\%().

[55] S. M. Walters, "A new direction for Broadband ISDN," IEEE Conumunications Magazine, pp. 39-42, Sep. 199 !.

[56] P. Wayner, "Smart memories," BYTE magazine, pp. 147-152. Mar. 1991

[57] M. R. Wernik and E. A. Munter, "Broadband public network and switch architecture," IEEE Communications Magazine, pp. 83-89. Jan. 1991. 

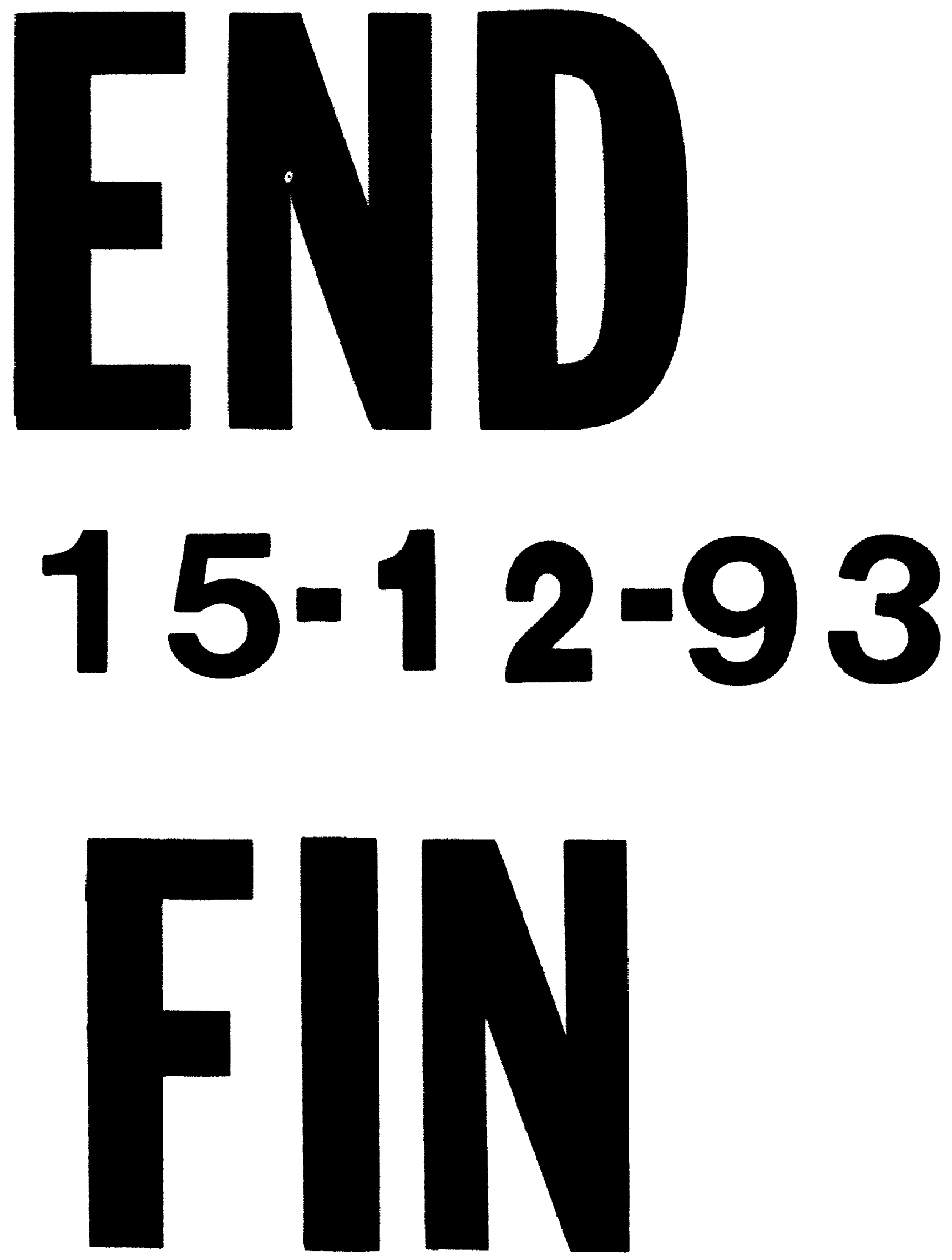